University of Nebraska - Lincoln

DigitalCommons@University of Nebraska - Lincoln

Timothy J. Gay Publications

Research Papers in Physics and Astronomy

May 1985

\title{
Experimental study of spin-exchange effects in elastic and ionizing collisions of polarized electrons with polarized hydrogen atoms
}

G. D. Fletcher

J.W. Gibbs Laboratory, Yale University, New Haven, Connecticut

M. J. Alguard

J.W. Gibbs Laboratory, Yale University, New Haven, Connecticut

Timothy J. Gay

University of Nebraska - Lincoln, tgay1@unl.edu

V. W. Hughes

J.W. Gibbs Laboratory, Yale University, New Haven, Connecticut

P.F. Wainwright

J.W. Gibbs Laboratory, Yale University, New Haven, Connecticut

See next page for additional authors

Follow this and additional works at: https://digitalcommons.unl.edu/physicsgay

Part of the Physics Commons

Fletcher, G. D.; Alguard, M. J.; Gay, Timothy J. ; Hughes, V. W.; Wainwright, P.F.; Lubell, M. S.; and Raith, W. , "Experimental study of spin-exchange effects in elastic and ionizing collisions of polarized electrons with polarized hydrogen atoms" (1985). Timothy J. Gay Publications. 12.

https://digitalcommons.unl.edu/physicsgay/12

This Article is brought to you for free and open access by the Research Papers in Physics and Astronomy at DigitalCommons@University of Nebraska - Lincoln. It has been accepted for inclusion in Timothy J. Gay Publications by an authorized administrator of DigitalCommons@University of Nebraska - Lincoln. 


\section{Authors}

G. D. Fletcher, M. J. Alguard, Timothy J. Gay, V. W. Hughes, P.F. Wainwright, M. S. Lubell, and W. Raith 
Phys. Rev. A 31, 2854 - 2884 (1985) [Issue 5 - May 1985]

\section{Experimental study of spin-exchange effects in elastic and ionizing collisions of polarized electrons with polarized hydrogen atoms}

G. D. Fletcher, M. J. Alguard, T. J. Gay, V. W. Hughes, and P. F. Wainwright

J.W. Gibbs Laboratory, Yale University, New Haven, Connecticut 06520

M. S. Lubell

J.W. Gibbs Laboratory, Yale University, New Haven, Connecticut 06520 and

Physics Department, The City College of the City University of New York, New

York, New York 10031

W. Raith

J.W. Gibbs Laboratory, Yale University, New Haven, Connecticut 06520 and University of Bielefeld, D-4800 Bielefeld, Federal Republic of Germany

Received 15 October 1984

Using crossed beams of polarized electrons and polarized hydrogen atoms we have investigated the effect of spin exchange on $90^{\circ}$ elastic scattering from 4.4 to $30.3 \mathrm{eV}$ and impact ionization from 14.1 to $197.0 \mathrm{eV}$. Our results suggest that the range of validity of various theoretical approximation methods is more restricted than had been assumed previously.

(C)1985 The American Physical Society

URL: http://link.aps.org/abstract/PRA/v31/p2854

DOI: 10.1103/PhysRevA.31.2854

PACS: 34.80.-i, 29.25.-t, 29.75.+x 


\title{
Experimental study of spin-exchange effects in elastic and ionizing collisions of polarized electrons with polarized hydrogen atoms
}

\author{
G. D. Fletcher, ${ }^{*}$ M. J. Alguard, ${ }^{\dagger}$ T. J. Gay, ${ }^{\ddagger}$ V. W. Hughes, and P. F. Wainwright ${ }^{\S}$ \\ J.W. Gibbs Laboratory, Yale University, New Haven, Connecticut 06520
}

M. S. Lubell

J.W. Gibbs Laboratory, Yale University, New Haven, Connecticut 06520

and Physics Department, The City College of the City University of New York, New York, New York 10031

\author{
W. Raith \\ J.W. Gibbs Laboratory, Yale University, New Haven, Connecticut 06520 \\ and University of Bielefeld, D-4800 Bielefeld, Federal Republic of Germany
}

(Received 15 October 1984)

\begin{abstract}
Using crossed beams of polarized electrons and polarized hydrogen atoms we have investigated the effect of spin exchange on $90^{\circ}$ elastic scattering from 4.4 to $30.3 \mathrm{eV}$ and impact ionization from 14.1 to $197.0 \mathrm{eV}$. Our results suggest that the range of validity of various theoretical approximation methods is more restricted than had been assumed previously.
\end{abstract}

\section{INTRODUCTION}

\section{A. Motivation}

During the last two decades, beams of polarized particles have found increasing application in a wide range of physics disciplines. While their actual use is relatively new, their potential has been recognized for many years. ${ }^{1}$ In addition to providing the primary access to the details of spin-dependent phenomena, polarization measurements can often circumvent the problems created by certain systematic effects thereby leading to an improvement in the precision of an experiment. $^{2}$

The earliest discussion of methods for producing polarized electrons in particular can be found in the work of Fues and Hellmann, ${ }^{3}$ published in 1930. It is remarkable that the first sources of polarized electrons, based on processes that they suggested, were not realized until almost 40 years later. ${ }^{4,5}$ Within the last 15 years, however, polarized-electron-beam technology has progressed rapidly. ${ }^{6,7}$ Indeed the last ten years have witnessed the use of polarized electrons in experiments in high-energy, ${ }^{8} \mathrm{nu}$ clear, ${ }^{9}$ and solid-state ${ }^{10,11}$ physics as well as atomic physics. ${ }^{12-24}$ This paper deals specifically with experimental studies of spin exchange in electron-hydrogen collisions, the most fundamental of all electron-atom systems.

Atomic collision theory finds its major interest in the development of approximation methods to handle the many-body problem involving long-range forces and has extensive applications to astrophysics, plasma physics, and chemical physics. From a theoretical perspective, atomic hydrogen is the ideal atomic system for tests of calculational methods, since the wave functions of hydrogen are known with virtual certainty. It is for this reason that even within the restricted scope of electron-atom collisions, several hundred theoretical papers have been published within the last 20 years on the subject of electron- hydrogen scattering.

Experimental studies of the electron-hydrogen system, however, have been relatively rare, since the problem of molecular contamination and the difficulty of obtaining high atom densities make such investigations arduous tasks at best. Nonetheless, by the mid 1970s, several detailed electron-hydrogen experiments had been carried out over a wide kinematic range and for many scattering channels. ${ }^{25-30}$ The agreement between these experiments and the sophisticated numerical calculations made possible by the development of fast computers with large-scale memories was so impressive that many theorists and experimentalists began to direct their efforts toward collisions involving molecules and complex atoms.

Ironically, however, new experimental techniques were just coming of age which promised to provide the opportunity for probing scattering amplitudes in detail, rather than relying on averages over spin and angular momentum states. This paper describes the first measurements obtained with one of these techniques, the application of polarized beams to electron-hydrogen scattering. The consequences of these measurements place significant restrictions on the applicability of many theoretical methods previously accepted as valid over wide dynamical ranges. Such restrictions have been suggested by earlier brief reports of the measurements, ${ }^{16-18,23,24}$ comprising $90^{\circ}$ elastic scattering from 4.4 to $30.3 \mathrm{eV}$ and impact ionization from 14.1 to $197.0 \mathrm{eV}$.

Why polarization measurements should be so revealing has been discussed extensively in a number of publications. ${ }^{31-34}$ In general, electron-atom scattering experiments that are performed with unpolarized incident beams and that lack either any polarization analysis, or, in the case of reactive collisions, any electron-photon or electron-electron coincidence analysis, result in the determination of a cross section $d \bar{\sigma} / d \Omega$, that is averaged over initial spin states, summed over final spin states, and in 
the case of excitation, summed over final angularmomentum sublevels. Here, for simplicity, we assume that initially, the atom is in an $S$ state. Then for a low- $Z$ valence- 1 atom, $d \bar{\sigma} / d \Omega$ is just the weighted average of the singlet and triplet cross sections, or ${ }^{35}$

$$
\frac{d \bar{\sigma}}{d \Omega}=\frac{1}{4}|f+g|^{2}+\frac{3}{4}|f-g|^{2},
$$

where $s=f+g$ is the singlet amplitude, $t=f-g$ is the triplet amplitude, and $f$ and $g$ are, respectively, the direct and exchange amplitudes. Since $d \bar{\sigma} / d \Omega$ contains sums and averages of individual amplitudes, it is clear that potentially some sensitivity may be lost in testing theoretical methods if only $d \bar{\sigma} / d \Omega$ is determined.

On the other hand, if experiments are performed with polarized beams in which the spins of the incident free electron and target valence electron are either antiparallel $(\uparrow \downarrow)$ or parallel $(\uparrow \uparrow)$, a cross-section asymmetry $\boldsymbol{A}$ can be measured that is defined by

$$
A=\frac{d \sigma(\uparrow \downarrow) / d \Omega-d \sigma(\uparrow \uparrow) / d \Omega}{d \sigma(\uparrow \downarrow) / d \Omega+d \sigma(\uparrow \uparrow) / d \Omega}
$$

It can be shown that in terms of $f$ and $g$ or, alternatively, in terms of $s$ and $t$, the expression for $A$ reduces to

$$
A=\frac{\operatorname{Re}\left(f^{*} g\right)}{d \bar{\sigma} / d \Omega}=\frac{|f||g| \cos \theta}{d \bar{\sigma} / d \Omega}=\frac{1-r}{1+3 r},
$$

where $\theta$ is the relative phase of $f$ and $g$ and $r=|t|^{2} /|s|^{2}$. From the form of Eq. (3) it is clear that the measurement of $A$ results in the determination of the interference between the direct and exchange amplitudes, provided $d \bar{\sigma} / d \Omega$ is already known. Only in the region of resonances can such information be gleaned in the absence of polarization measurements. ${ }^{29}$

Within the context of experiments restricted to two polarization devices (sources or polarimeters), the measurement described above is but one of six that are possible for nonreactive scattering. The complete set of experiments is illustrated schematically in Fig. 1 where $\mathbf{P}_{e}$ and $\mathbf{P}_{e}^{\prime}$ are the polarization vectors associated, respectively, with the incident and scattered electrons and $\mathbf{P}_{a}$ and $\mathbf{P}_{a}^{\prime}$ are the polarization vectors associated with the incident and scattered atoms. Of the six experiments shown, however, the two in each row provide identical information. This information can be expressed in the form of three asymmetry parameters, denoted by $A, A^{\prime}$, and $A^{\prime \prime}$ for rows 1 , 2 , and 3 , respectively, where $A$ has already been expressed in more elementary form by Eq. (3). The two remaining parameters can easily be shown ${ }^{18}$ to be given by the expressions

$$
A^{\prime}=|g|^{2}(d \bar{\sigma} / d \Omega)^{-1}-1
$$

and

$$
A^{\prime \prime}=|f|^{2}(d \bar{\sigma} / d \Omega)^{-1}-1 .
$$

From Eqs. (1) and (3)-(5) it can be seen that $A, A^{\prime}$, and $A^{\prime \prime}$ are not linearly independent but rather are related by the equation

$$
A-A^{\prime}-A^{\prime \prime}=1 \text {. }
$$

As a consequence, only two experiments from any two rows represented in Fig. 1 are truly independent.

It might appear from the foregoing discussion that the performance of any two of these independent experiments together with the measurement of $d \bar{\sigma} / d \Omega$ is sufficient to determine the three scattering parameters $|f|,|g|$, and $\theta$. In fact, however, an ambiguity in the sign of $\theta$ still remains, since only $\cos \theta$ is determined. This ambiguity can be removed through the measurement of a polarization rotation of either the electrons or the atoms induced by the collision. If, for example, a longitudinally polarized electron beam traveling along the $x$ axis is incident on a longitudinally polarized atom beam traveling along the $y$ axis, the $z$ component of the polarization of the scattered electrons, $\left(P_{e}^{\prime}\right)_{z}$, is given by

$$
\left(P_{e}^{\prime}\right)_{z}=P_{e} P_{a} \frac{|f||g| \sin \theta}{d \bar{\sigma} / d \Omega}
$$

where $\mathbf{P}_{e}=\boldsymbol{P}_{e} \hat{\mathbf{x}}$ and $\mathbf{P}_{a}=\boldsymbol{P}_{a} \hat{\mathbf{y}}$ are the incident electron and atom polarizations, respectively. The analogous expression for the asymmetry measured in experiment (1) of Fig. 1 for nonunity incident polarization follows directly from Eq. (3) and is given by

$$
\Delta^{\prime}=P_{e} P_{a} \frac{|f||g| \cos \theta}{d \bar{\sigma} / d \Omega} .
$$

Thus from experiments described by Eqs. (7) and (8), the relative phase $\theta$ can be specified uniquely. While modern techniques allow any of the seven experiments previously described to be performed, experiment (1) of Fig. 1, resulting in the determination of $A$, poses the least difficulty for the case of electron-hydrogen collisions. It is this experiment that we performed for $90^{\circ}$ elastic $e^{-}-\mathrm{H}$ scattering, as well as its natural extension for $e^{-}-\mathrm{H}$ impact ionization.

For the case of impact ionization we define an analogous asymmetry $A_{I}$ by the relation

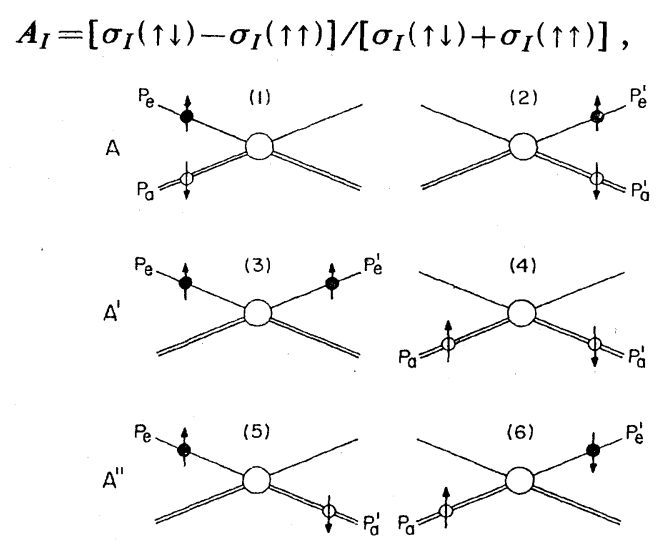

FIG. 1. Nonreactive "second-generation" scattering experiments. Particles are incident from the left with electrons denoted by single lines and atoms by double lines. The circles, solid for electrons and open for atoms, indicate measured beams with the measured quantities denoted by $P_{e}$ and $P_{a}$ for the electron and atom polarizations, respectively, in the incoming channels. Primes indicate quantities measured in the outgoing channels. Asymmetries measured in a given row are specified by $A, A^{\prime}$, and $A^{\prime \prime}$ in accordance with Eqs. (3), (4), and (5). 
where $\sigma_{I}(\uparrow \downarrow)$ and $\sigma_{I}(\uparrow \uparrow)$ now are the total ionization cross sections for the antiparallel and parallel spin orientations, respectively. Continuing the analogy with the case of elastic scattering we observe that $A_{I}$ can be expressed in terms of $r_{I}$, the ratio of triplet to singlet total ionization cross sections, as

$$
A_{I}=\left(1-r_{I}\right) /\left(1+3 r_{I}\right),
$$

or in terms of the respective direct and exchange amplitudes, $f\left(\mathbf{k}_{1}^{\prime}, \mathbf{k}_{2}^{\prime}\right)$ and $g\left(\mathbf{k}_{1}^{\prime}, \mathbf{k}_{2}^{\prime}\right)$, as

$$
A_{I}=\sigma_{I}^{\text {int }} / \bar{\sigma}_{I}
$$

where

$$
\begin{gathered}
\sigma_{I}^{\text {int }}=\frac{1}{2} \int_{0}^{E} d E_{1}^{\prime} \iint \frac{k_{1}^{\prime} k_{2}^{\prime}}{k_{1}} \operatorname{Re}\left[f^{*}\left(\mathbf{k}_{1}^{\prime}, \mathbf{k}_{2}^{\prime}\right) g\left(\mathbf{k}_{1}^{\prime}, \mathbf{k}_{2}^{\prime}\right)\right] \\
\times d \hat{\mathbf{k}}_{1}^{\prime} d \hat{\mathbf{k}}_{2}^{\prime}
\end{gathered}
$$

and

$$
\begin{gathered}
\bar{\sigma}_{I}=\frac{1}{2} \int_{0}^{E} d E_{1}^{\prime} \iint \frac{k_{1}^{\prime} k_{2}^{\prime}}{k_{1}}\left(\frac{1}{4}|f+g|^{2}+\frac{3}{4}|f-g|^{2}\right) \\
\times d \hat{\mathbf{k}}_{1}^{\prime} d \hat{\mathbf{k}}_{2}^{\prime} .
\end{gathered}
$$

Here $\mathbf{k}_{1}, \mathbf{k}_{1}^{\prime}$, and $\mathbf{k}_{2}^{\prime}$ are, respectively, the momenta of the incident, scattered, and ejected electrons, $E_{1}^{\prime}$ is the energy of the scattered electron, and $E+\frac{1}{2}$ is the energy of the incident electron, all quantities being given in atomic units. ${ }^{16}$ We conclude our preliminary comments on the ionization asymmetry by pointing out that $\sigma_{I}^{\text {int }}$ can also be written as ${ }^{36}$

$$
\sigma_{I}^{\text {int }}=\sigma_{I}^{*}-\bar{\sigma}_{I},
$$

where $\sigma_{I}^{*}$ is the total ionization cross section calculated in the absence of exchange. This relation is extremely useful for extracting asymmetry information from a number of older calculations.

We have organized the balance of this paper to present in Sec. IB a brief theoretical review of electron-hydrogen scattering, in Sec. II a detailed description of the experimental apparatus, in Sec. III an explanation of the experimental procedure, in Sec. IV a description of the data analysis, and in Sec. $\mathrm{V}$ a discussion of our results together with their possible implications for theoretical methods as well as future experimental efforts.

\section{B. Theoretical background}

In the following paragraphs we present the general formulation of the $e^{-}-\mathrm{H}$ collision problem along with short descriptions of the important approximation methods. We discuss the strengths and weaknesses of these methods and compare their results with elastic and ionization measurements. Since we use the detailed results of atomic scattering theory we refer the reader to textbook discussions by Mott and Massey, ${ }^{35}$ Geltman, ${ }^{37}$ Burke, ${ }^{38}$ Joachain, ${ }^{39}$ and Bransden ${ }^{40}$ for further information. We also point out that the approximation methods have been reviewed recently in several excellent articles. ${ }^{41-45}$

In the theoretical formulation of the scattering of electrons by hydrogen atoms (and other light one-electron atoms), relativistic effects such as the spin-orbit interaction are generally neglected. Since the Hamiltonian describing the incident and atomic electrons (the nucleus considered to be infinitely heavy) contains kinetic and electrostatic terms only, the nonrelativistic wave equation takes the form (in atomic units)

$$
\begin{aligned}
\left(-\frac{1}{2} \nabla_{1}^{2}-\frac{1}{2} \nabla_{2}^{2}\right. & -\frac{1}{r_{1}}-\frac{1}{r_{2}} \\
& \left.+\frac{1}{\left|\mathbf{r}_{1}-\mathbf{r}_{2}\right|}-E\right] \psi\left(\mathbf{r}_{1}, s_{1} ; \mathbf{r}_{2}, s_{2}\right)=0,
\end{aligned}
$$

where $\left(\mathbf{r}_{1}, s_{1}\right)$ and $\left(\mathbf{r}_{2}, s_{2}\right)$ are the spatial and spin coordinates of the incident and atomic electrons, respectively, and $\nabla_{1}^{2}$ and $\nabla_{2}^{2}$ are the respective kinetic energy operators. Since the Hamiltonian does not depend explicitly on the spin coordinates, the two-electron wave function can be written as a product of spatial and spin functions:

$$
\psi\left(\mathbf{r}_{1}, s_{1} ; \mathbf{r}_{2}, s_{2}\right)=\psi\left(\mathbf{r}_{1}, \mathbf{r}_{2}\right) \chi\left(s_{1}, s_{2}\right) .
$$

The requirement of exchange antisymmetry on the total wave function imposed by Fermi-Dirac statistics then reduces to a combination of a symmetric spatial wave function with an antisymmetric (singlet) spinor or alternatively an antisymmetric spatial wave function with a symmetric (triplet) spinor.

In the time-independent description of the scattering problem the asymptotic form of the spatial wave functions must represent an incoming plane wave and an outgoing spherical scattered wave. Since two appropriately symmetrized spatial wave functions can be formed, it should be expected that in the asymptotic limit, scattering amplitudes for the symmetric spatial wave function, corresponding to singlet scattering, and the antisymmetric spatial wave function, corresponding to triplet scattering, will be different. The singlet and triplet amplitudes can also be expressed in terms of the direct and exchange amplitudes, as was indicated in Sec. IA. The collisions described by Eqs. (17)-(19) below illustrate the use of these amplitudes. In each collision, the atom is assigned an arbitrary spin direction, and the scattering of electrons is considered with spins either parallel or antiparallel to the atomic spin:

\begin{tabular}{ll} 
Experiment & Cross section \\
\hline$e(\downarrow)+A(\uparrow) \rightarrow e(\downarrow)+A(\uparrow)$ & $|f|^{2}$ \\
$e(\downarrow)+A(\uparrow) \rightarrow e(\uparrow)+A(\downarrow)$ & $|g|^{2}$ \\
$e(\uparrow)+A(\uparrow) \rightarrow e(\uparrow)+A(\uparrow)$ & $|f-g|^{2}$
\end{tabular}

In Eq. (17) the process illustrated is direct scattering, denoted by the amplitude $f$; in Eq. (18) the interchange of the incident and atomic electrons leads to the exchange amplitude $g$; in the last experiment, the indistinguishability of electrons results in the triplet amplitude $f-g$, the minus sign arising from the requirements of Fermi-Dirac statistics. Generally, theoretical approximations provide values of the singlet and triplet, or equivalently, the direct 
and exchange amplitudes.

Two main approaches exist for the solution of Eq. (15)_-perturbative and variational. Further, for the discussion of elastic scattering, the energy range of the incident electrons can be divided into three regions: low energy, meaning energies below the first excitation threshold at $10.2 \mathrm{eV}$; intermediate energy ranging from 10.2 to $\sim 100 \mathrm{eV}$; and high energy, implying energies above 100 $\mathrm{eV}$ where the effects of exchange and correlation between the electrons during the collision are less important.

In the low-energy region, in which only elastic scattering is energetically allowed, variational techniques such as the Hulthén-Kohn and inverse Kohn methods, using appropriate trial functions, have been employed to calculate what are generally regarded as the most accurate singlet and triplet phase shifts for $s$ waves, ${ }^{46-49} p$ waves, ${ }^{49-51}$ and $d$ waves. $^{49,52}$ Less accurate $d$ - and $f$-wave phase shifts have been calculated using a pseudostate basis. ${ }^{53}$ At high energies, exchange effects are usually neglected completely and a perturbation approach is taken, since reasonably rapid convergence of the perturbation series can be expected. Extensions of the Born approximation, such as the distorted-wave second-Born approximation, ${ }^{54}$ the second-order ${ }^{55-60}$ and third-order ${ }^{61}$ optical potential methods, and the eikonal-Born series, ${ }^{42,62-64}$ as well as the Glauber ${ }^{65-67}$ and modified Glauber ${ }^{68}$ approximations, the two-potential eikonal approximation, ${ }^{69}$ and the Faddeev-Watson multiple scattering expansion ${ }^{70}$ have been used with moderate success in electron-hydrogen elastic scattering calculations.

In the intermediate-energy region the problem is considerably more complicated, since exchange effects cannot be neglected and there can exist a large, if not infinite, number of open channels to which the initial state can couple. Typical approaches to calculations in this energy region are extensions and modifications of methods applicable to either the low- or high-energy regions. With the low-energy approach, ${ }^{41}$ the total wave function can be expanded, for example, in the form

$$
\begin{aligned}
& \psi\left(\mathbf{r}_{1}, s_{1} ; \mathbf{r}_{2}, s_{2}\right) \\
& =\left[1+(-1)^{s} \boldsymbol{P}_{12}\right]\left[\sum_{i=0}^{p} \phi_{i}\left(\mathbf{r}_{2}, s_{2}\right) F_{i}\left(\mathbf{r}_{1}, s_{1}\right)\right. \\
& \left.+\sum_{i=p+1}^{q} \bar{\phi}_{i}\left(\mathbf{r}_{2}, s_{2}\right) F_{i}\left(\mathbf{r}_{1}, s_{1}\right)\right] \\
& +\sum_{i=1}^{r} a_{i} \chi_{i}\left(\mathbf{r}_{1}, s_{1} ; \mathbf{r}_{2}, s_{2}\right),
\end{aligned}
$$

where $\phi_{i}$ and $\bar{\phi}_{i}$ are atomic eigenstates and pseudostates, respectively, $\chi_{i}$ are square-integrable short-range correlation functions, already antisymmetrized, $S$ is the total spin, $P_{12}$ is the operator which interchanges the spatial coordinates of the two electrons, and $F_{i}$ and $a_{i}$ are expansion functions. Projection of the wave equation, Eq. (15), with this wave function onto the eigenstates $\phi_{i}$, the pseudostates $\bar{\phi}_{i}$, and the correlation functions $\chi_{i}$, produces integro-differential equations for the functions $F_{i}\left(\mathbf{r}_{1}, s_{1}\right)$ coupled to linear simultaneous equations for the $a_{i}$ coefficients. The asymptotic form of the solutions $F_{i}\left(\mathbf{r}_{1}, s_{1}\right)$ of these coupled equations then yields the scattering amplitudes.

The various approximation methods employed in the low-energy region or in the low-energy approach to the intermediate-energy region derive their respective names from the types of terms included in Eq. (20). In the close-coupling approximation ${ }^{71-73}$ only a few atomic eigenstates $\phi_{i}$ (usually all the open channels and a few closed channels) are used. The inclusion of correlation functions in addition to the close-coupling eigenstates, called the correlation approximation, ${ }^{74}$ tends to accelerate convergence to a solution when the atomic eigenstates are closely spaced in energy. To account for the infinite number of eigenstates which are not included in the first term, but are needed to describe fully the polarizability of the hydrogen atom, several approaches substitute a small number of pseudostates $\bar{\phi}_{i}$ which provide more adequately for the long-range interaction..$^{30,75-77}$ (The polarizedorbital method for example, can be included in this category. ${ }^{78,79}$ ) The solutions to the equations resulting from all of these approaches rely on the use of numerical techniques, among them variational ${ }^{80,81}$ and $R$-matrix methods. ${ }^{82,83}$

For all low-energy approximation approaches to the intermediate-energy region one problem remains outstanding: Precisely how accurate is it to replace the complete atomic eigenstate expansion by a finite sum of atomic states, pseudostates, and correlation functions? It is well known, for example, that unphysical thresholds may arise from the opening of pseudochannels, while "ghost resonances" may appear with the use of correlation functions. On the other hand, if high-energy approximation approaches are used in the intermediate-energy region, another problem arises; namely, the proper treatment of exchange. ${ }^{84}$ Various approximations, including BornOppenheimer, ${ }^{85}$ Born exchange, ${ }^{62}$ and Ochkur, ${ }^{86}$ have been used, and in some cases exchange integrals have been evaluated, but with various degrees of success. ${ }^{67,68,87-93}$

In the case of differential elastic scattering, calculations have been subjected to scrutiny by absolute spin-averaged cross-section measurements obtained by Williams ${ }^{28-30}$ for angles between $10^{\circ}$ and $150^{\circ}$ and energies from 0.5 to 680 $\mathrm{eV}$. Additional experimental data have been provided by Teubner and co-workers ${ }^{27}$ who measured $e^{-}-\mathbf{H}$ spinaveraged cross sections relative to $e^{-}-\mathrm{H}_{2}$ cross sections for angles from $15^{\circ}$ to $135^{\circ}$ and energies from 9.4 to 200 $\mathrm{eV}$. The latter data in the range of 100 to $200 \mathrm{eV}$ were placed on an absolute scale with the aid of $e^{-}-\mathrm{H}_{2}$ crosssection measurements by van Wingerden et al. ${ }^{94}$

In the low-energy region, the measurements of Williams and Teubner confirm the predictions of the variational calculations and the similar predictions of the pseudostate close-coupling $\operatorname{method}^{75}$ and the polarized-orbital method, ${ }^{78}$ all of which account fully for the ground-state polarizability of the hydrogen atom. ${ }^{28}$ Close-coupling calculations $^{75}$ also account well for the positions and widths of resonances below the $n=2$ threshold. In the intermediate-energy region from 10 to $30 \mathrm{eV}$, the measurements lend credence to the pseudostate close-coupling calculations of Callaway and Wooten ${ }^{76}$ and Callaway ${ }^{30}$ except at large scattering angles $\left(\theta>120^{\circ}\right)$ for energies be- 
tween 20 and $26 \mathrm{eV}$. A recent calculation by Burke and his collaborators ${ }^{83}$ using the $R$-matrix method to solve pseudostate close-coupling equations also agrees well with measurements from 12 to $50 \mathrm{eV}$, while an earlier $1 S-\overline{2 P}$ pseudostate close-coupling calculation by the same group, ${ }^{77}$ carried out over a larger energy range, provides less good agreement below $50 \mathrm{eV}$ and at energies above 50 $\mathrm{eV}$ underestimates the cross section for small-angle scattering $\left(\theta \leq 40^{\circ}\right)$ by as much as $40 \%$.

At higher energies, most of the calculations based on the so-called high-energy methods underestimate differential cross sections at 100 and $200 \mathrm{eV}$, typical energies at which calculations are performed. The eikonal-Born series $^{63}$ and the modified Glauber approximation, ${ }^{68}$ along with the $1 S-\overline{2 P}$ calculations referred to above, give the best agreement with experiment, being typically $15 \%$ below the measured values for intermediate angles at 100 and $200 \mathrm{eV}$. By contrast the Faddeev-Watson multiple scattering expansion ${ }^{70}$ overestimates the cross section from 50 to $200 \mathrm{eV}$ by more than an order of magnitude in some cases. On the other hand, at $400 \mathrm{eV}$ the eikonalBorn series ${ }^{63}$ and the distorted-wave second Born approximation $^{54}$ agree well with the measurements, and at $680 \mathrm{eV}$ the first Born approximation produces results within the experimental uncertainty of the measurements. ${ }^{28}$

When high-energy methods are extended into the intermediate-energy region, good agreement is obtained at $50 \mathrm{eV}$ with the eikonal-Born series, the distorted-wave second Born approximation, and the second-order potential method. ${ }^{60}$ However, at $30 \mathrm{eV}$ the latter two methods (eikonal-Born series results have not been published at 30 $\mathrm{eV})$ predict cross sections that are too large by as much as a factor of 1.8 at small angles $\left(\theta<40^{\circ}\right)$. It has been suggested that the forward-angle behavior of these methods at lower energies results from an inadequate treatment of exchange, ${ }^{84,92,95}$ with a justifiable inference that measurements of the elastic asymmetry $A$ in the intermediateenergy region should be of considerable value.

In the case of ionization, one energy region of particular interest is that just above threshold. Here the motions of the scattered and ejected electrons are so highly correlated that the independent-electron model is inadequate. In 1953, Wannier ${ }^{96}$ derived a threshold law, or energy dependence of the cross section, from detailed arguments involving phase space and classical equations of motion. He showed that near threshold the outgoing electrons are equidistant from the nucleus on radii $180^{\circ}$ apart and the energy distribution of each electron is uniform, with the consequence that the total ionization cross section obeys the threshold law $\sigma \propto E^{1.127}$, where $E$ is the total energy of the outgoing electrons. Wannier's prediction of a nonlinear energy dependence was verified in 1968 by McGowan and Clark ${ }^{97}$ and in 1974 by Cvejanović and $\operatorname{Read}^{98}$ from direct measurements of electron impact ionization and very recently again by Bryant and his coworkers $^{99}$ from measurements of two-electron photoionization of $\mathbf{H}^{-}$.

The classical Wannier threshold law was derived quantum mechanically by $\mathrm{Rau}^{100}$ and Peterkop ${ }^{101}$ for ${ }^{1} S^{e}$ states and subsequently extended by Roth ${ }^{102}$ and Klar and Schlecht ${ }^{103}$ to higher angular-momentum states. In their paper, Klar and Schlecht considered the role of spin explicitly for the first time and concluded that at threshold singlet scattering should follow the Wannier law, while triplet scattering should be suppressed by an $E^{3.881}$ dependence. As a consequence they predicted that at threshold the ionization cross-section asymmetry $A_{I}$ should be unity, a value not observed in either the hydrogen experiment $^{16}$ or in similar experiments with alkali-metal atoms. ${ }^{19,21,22}$ Subsequently, Greene and $\mathrm{Rau}^{104}$ showed that the conclusions of Klar and Schlecht were incorrect. Instead of general triplet state suppression, Greene and Rau found that only two even-parity (e) states, the ${ }^{3} S^{e}$ and ${ }^{1} p^{e}$, were suppressed. Moreover they concluded that states with total angular momentum $L=1$ and 2 should contribute significantly to the cross section even at threshold, implying that the threshold value of $A_{I}$ can differ substantially from unity.

Even more recently, it has been suggested that the Wannier behavior itself might be in error. Using an argument that even near threshold the two outgoing electrons could have significantly different energies, Temkin ${ }^{105}$ derived a modulated linear threshold law. To date, however, no experiments have revealed any deviations from the Wannier law in the energy range investigated. Very high-resolution measurements of $A_{I}$ extremely close to threshold, however, might yet shed some light on this issue.

In a more general context for ionization, it should be noted that a special case occurs, which in principle should allow the exchange amplitude to be evaluated precisely. Since the two outgoing electrons are indistinguishable, $f$ and $g$ obey the relation ${ }^{36}$

$$
g\left(\mathbf{k}_{1}^{\prime}, \mathbf{k}_{2}^{\prime}\right)=f\left(\mathbf{k}_{2}^{\prime}, \mathbf{k}_{1}^{\prime}\right)
$$

in which $\mathbf{k}_{1}^{\prime}$ and $\mathbf{k}_{2}^{\prime}$ are the momenta of the two escaping electrons. As a practical matter, however, Eq. (21) is of limited use, since some variant of the Born approximation is used in most ionization calculations, ${ }^{85}$ with the consequence that $f$ is not known exactly and Eq. (21) no longer holds for the approximated amplitudes. In spite of the difficulties raised by exchange, for ionization of hydrogen from threshold to more than $100 \mathrm{eV}$, calculations (mostly Born exchange) of the spin-averaged total cross section agree reasonably well with the experimental results. ${ }^{25,106}$

\section{EXPERIMENTAL APPARATUS}

\section{A. Overview}

We now consider the experimental determination of the cross-section asymmetry for $90^{\circ}$ elastic scattering and total impact ionization. While all of the measurements reported here were performed with the same basic apparatus, significant modifications implemented following the early ionization ${ }^{16}$ and exploratory elastic ${ }^{18}$ measurements allowed us recently to study these cross-section asymmetries more thoroughly. ${ }^{23,24}$ In the paragraphs which follow, a description of the apparatus with relevant experimental details is given for both the early ${ }^{16}$ and recent $^{23,24}$ measurements.

The experimental layout is shown schematically in Fig. 2. Longitudinally polarized electrons were produced in a 


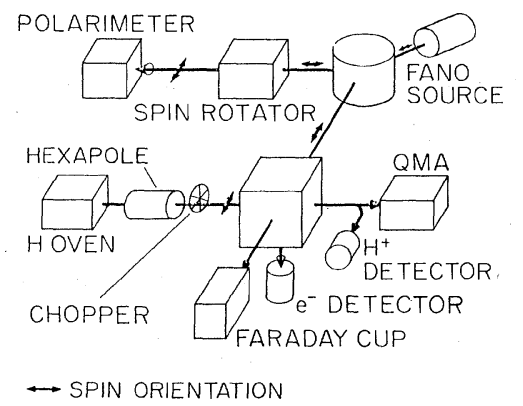

FIG. 2. Block diagram of the experiment. Spin orientations of the beams are indicated by darkened arrows. Both electron and atom polarizations could be reversed easily. QMA refers to the quadrupole mass analyzer.

Fano-effect ${ }^{17}$ source, extracted at $1 \mathrm{keV}$ energy, and deflected by $\pm 45^{\circ}$ in a vertical magnetic field. For polarization measurements, the electrons were deflected toward the Mott polarimeter, which was preceded by a Wien fil$\operatorname{ter}^{107}$ to rotate the electron spin to the transverse direction prior to acceleration to $\sim 100 \mathrm{keV}$ for Mott scattering polarization analysis. ${ }^{17,108}$ For scattering from atomic hydrogen the electron beam was deflected toward the hydrogen beam, the two beams intersecting at $90^{\circ}$ in the interaction region. Immediately prior to the interaction region the electrons were decelerated to the desired energy. Electrons elastically scattered at $90^{\circ}$ were detected by an electron multiplier, while electrons which remained in the beam were collected in a Faraday cup. The hydrogen beam line consisted of a tungsten oven for thermal dissociation of $\mathrm{H}_{2}$, a hexapole magnet for state selection, a tuning-fork chopper for intermittent background measurements, and, following intersection with the electron beam, a quadrupole mass analyzer (QMA) for monitoring the atomic and molecular components of the beam. Ions created by electron impact were detected by a second electron multiplier located downstream from the interaction region along the hydrogen beam.

With the exception of the Wien filter and Mott polarimeter chambers, which were constructed of aluminum and evacuated by an oil diffusion pump, the original vacuum system consisted of differentially pumped bakeable stainless-steel ultrahigh-vacuum chambers using mercury diffusion pumps with Freon-cooled chevron baffles and liquid-nitrogen $\left(\mathrm{LN}_{2}\right)$ cold traps. The interaction region chamber also included a titanium sublimation pump and typically attained a base pressure of $5 \times 10^{-10}$ Torr following $24 \mathrm{~h}$ of baking.

For the recent measurements, the vacuum system was modified by the replacement of the mercury diffusion pumps and $\mathbf{L N}_{2}$ traps in the first two chambers of the hydrogen beam (hydrogen source and differential pumping chambers) with oil diffusion pumps in order to increase the pumping speed in this region. These changes permitted approximately a factor of 2 increase in the molecular hydrogen leak rate into the source, with a corresponding increase in atomic hydrogen density in the interaction region. No effect due to hydrocarbon deposit on electrostatic elements in the interaction region was detected.

\section{B. Electron beam}

\section{Source}

Photoionization of an unpolarized cesium atomic beam by circularly polarized light of energy just above the photoionization threshold produces polarized electrons with the same helicity as that of the incident light. This effect, known as the Fano effect, ${ }^{109}$ is the basis of the source of longitudinally polarized electrons used in this work. ${ }^{17}$ The Fano source, shown in Fig. 3, consisted of a $\mathrm{Hg}-\mathrm{Xe}$ arc lamp and associated optics (providing $120 \mathrm{~mW}$ of circularly polarized light in the wavelength range 280-320 $\mathrm{nm}$ ) and a vacuum chamber with an effusive atomic cesium beam and electrostatic optics to extract photoelectrons emitted over $4 \pi$ solid angle. Important advantages of this source for our experiment were the high degree and optical reversibility of the electron polarization. Rotation by $90^{\circ}$ of either a dichroic linear polarizer or a zeroth-order

TABLE I. Experimental operating parameters for the recent work.

\begin{tabular}{|c|c|}
\hline \multicolumn{2}{|l|}{ Electron beam } \\
\hline \multicolumn{2}{|l|}{ Intensity } \\
\hline At source exit & $10 \mathrm{nA}$ average \\
\hline At interaction region & $2 \mathrm{nA}$ average \\
\hline Polarization, $P_{e}{ }^{\mathrm{a}}$ & $0.61-0.75( \pm 5 \%)$ \\
\hline Energy spread (FWHM) & $2.5 \mathrm{eV}$ \\
\hline Emittance at $1 \mathrm{keV}$ & $20 \mathrm{mrad} \mathrm{cm}$ \\
\hline Cs-oven capacity & $60 \mathrm{~g}$ \\
\hline Cs-oven lifetime & $\sim 40 \mathrm{~h}$ \\
\hline Cs-beam density & $10^{12} \mathrm{~cm}^{-3}$ \\
\hline Polarization reversal & Optical \\
\hline \multicolumn{2}{|l|}{ Hydrogen beam } \\
\hline Density at interaction region & $\sim 10^{9} \mathrm{~cm}^{-3}$ \\
\hline \multicolumn{2}{|l|}{ Polarization, $P_{H}^{\mathrm{b}}$} \\
\hline At high field & $0.99( \pm 1 \%)$ \\
\hline At low field & $0.50( \pm 4 \%)$ \\
\hline \multicolumn{2}{|l|}{ Fraction of elastic events, } \\
\hline Oven lifetime & $20-25 \mathrm{~h}$ \\
\hline Polarization reversal & Magnetic \\
\hline Collinearity factor, $\cos \alpha$ & $0.985( \pm 1.5 \%)$ \\
\hline Background gas pressure & $10^{-9}$ Torr \\
\hline \multicolumn{2}{|l|}{ Detection solid angle } \\
\hline Electrons & $0.15 \mathrm{sr}$ \\
\hline Ions & $4 \pi \mathrm{sr}$ \\
\hline \multicolumn{2}{|l|}{ Counting rates $(20.1 \mathrm{eV})$} \\
\hline Electrons & $2.6 \mathrm{~s}^{-1}$ \\
\hline Ions & $69 s^{-1}$ \\
\hline \multicolumn{2}{|l|}{ Signal-to-noise ratios $(20.1 \mathrm{eV})$} \\
\hline Electrons & 1.7 \\
\hline Ions & 9.1 \\
\hline
\end{tabular}

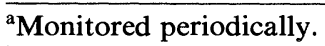

${ }^{\text {b}}$ Calculated, including hyperfine depolarization.

${ }^{\mathrm{c}}$ Monitored continuously. 


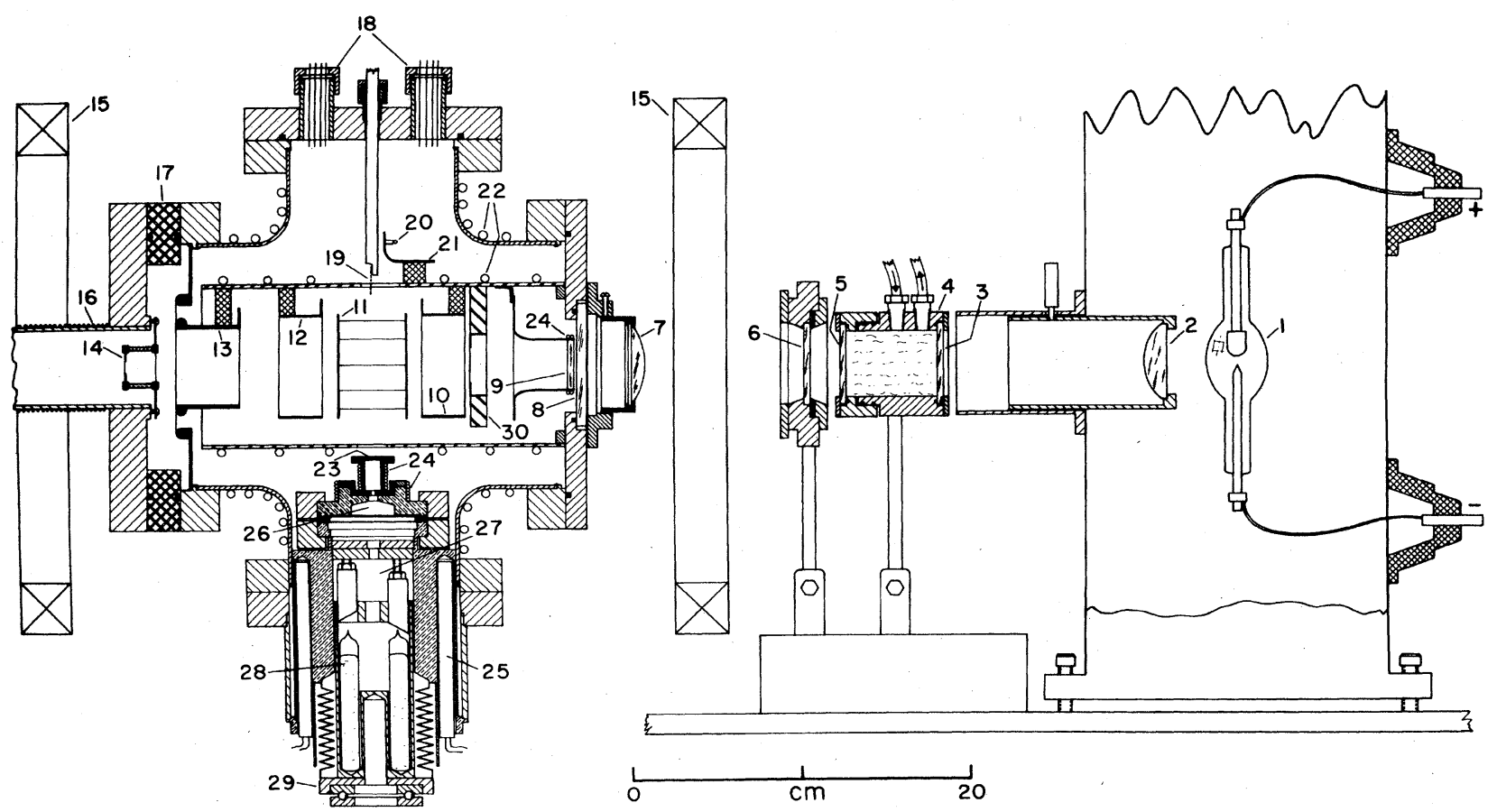

FIG. 3. Side-view scale drawing of the Fano effect polarized electron source showing the following elements: (1) 1000-W Hg-Xe cw arc lamp with Suprasil envelope (Hanovia 977B-1); (2) f/1.5 quartz lens; (3) Corning CSO-56 filter; (4) NiSO $_{4}$ absorption cell; (5) dichroic linear polarizer (3M Company 105 UV WRMR) on a 5-cm diameter rotatable quartz disc; (6)3000- $\AA$ zeroth-order quartz rotatable quarter-wave retardation plate; (7) f/3.0 quarts lens; (8) Suprasil vacuum window; (9) heated quartz disc to prevent Cs from fogging the window; (10) repeller electrode; (11) ionization region; (12) extractor electrode; (13) focusing electrode; (14) electron collimator; (15) Helmholtz coils to establish a fixed $\sim 200-\mathrm{mG}$ magnetic field in the ionization region colinear with the ionizing light; (16) beam pipe with solenoid; (17) Lucite insulating flange; (18) electrical feedthroughs; (19) stainless-steel mesh; (20) hot-wire surface ionization Cs-beam detector with ion collector (21); (22) Freon cooling pipes; (23) stainless-steel multicapillary orifice (Wintec Division, Brunswick Corp.); (24) Thermocoax heating coils (North American Phillips); (25) 12 300-W heaters; (26) upper oven chamber; (27) lower oven chamber; (28) six 10:g Pyrex ampoules of Cs metal (Kawecki Berylco Industries); (29) bellows mechanism for breaking ampoules; (30) Freon-cooled copper aperture. All vacuum parts were machined from stainless steel unless otherwise noted. Oven temperatures were monitored by Chromel-Alumel thermocouples (not shown). Also not shown is an externally operable Cs beamblocking flag.

quarter-wave retardation plate reversed the helicity of the incident light, thereby reversing the electron polarization. Typical operating parameters for the Fano source are given in Table I.

Extraction of the polarized electrons from the photoionization volume, where the light and cesium beams intersected at $90^{\circ}$, was accomplished by an $\sim 1.5-\mathrm{V} / \mathrm{cm}$ electric field in the presence of an $\sim 200-\mathrm{mG}$ magnetic field, both fields being parallel to the light direction. From computer modeling of the source electrode structure, these fields were found to provide efficient extraction of polarized electrons from the photoionization region while preventing the extraction of electrons photoemitted from surfaces. Spin-exchange collisions between slow polarized electrons and cesium atoms (beam density $\sim 10^{12} \mathrm{~cm}^{-3}$ ), believed to be the cause of the observed electron depolarization at lower electric fields, ${ }^{17}$ precluded the use of a larger magnetic field or a smaller electric field with its resultant smaller electron energy width.

Great care was required to prevent cesium deposition on the uv vacuum window (8). [The number here refers to element (8) of Fig. 3.] Initially a heated quartz disc (9) was placed behind the window. Later, shielding was improved by the addition of a Freon-cooled aperture (30). For test purposes, an unpolarized electron beam of $\sim 1$ $\mu \mathrm{A}$ intensity with an energy spread $<0.4-\mathrm{eV}$ full width at half maximum (FWHM) was produced by photoemission from a retractable stainless-steel mesh (19) positioned at the intersection of the light and cesium beams.

\section{Transport}

Electrons extracted from the Fano source were accelerated to $1 \mathrm{keV}$ to facilitate transport to either the hydrogen interaction region or the Mott polarimeter. For strong steering and focusing in the transport system magnetic fields were used, since they have a minimal effect on the longitudinal polarization of $1-\mathrm{keV}$ electrons. For weak electron-optical effects, electrostatic elements were used as a matter of convenience. Faraday cups and current sensors following major sections of the electron optics were incorporated for tuning and monitoring the electron beam. Typically $20 \%$ of the electrons leaving the 
Fano source were transported to the large-aperture Faraday cup downstream of the hydrogen interaction region.

\section{Polarimeter}

Elastic Mott scattering ${ }^{110}$ was used to measure the polarization of the electrons from the Fano source. After deflection by $45^{\circ}$ toward the polarimeter branch of the experiment, the longitudinally polarized electrons were first accelerated to $7 \mathrm{keV}$ (to minimize the effects of fringe fields) and then passed undeflected through a Wien filter spin rotator to precess the electron spin by $90^{\circ}$. The resulting transversely polarized electrons were further accelerated to $\sim 93 \mathrm{keV}(v / c=0.53)$ and scattered from a thin gold-foil target backed by a Formvar film on an aluminum frame. Figure 4 illustrates the geometry of the Mott polarimeter. The target wheel (6) contained four gold foils ranging in thickness from 27 to $62 \mu \mathrm{g} / \mathrm{cm}^{2}$, a bare Formvar film approximately $20 \mu \mathrm{g} / \mathrm{cm}^{2}$ thick, and a ${ }^{109} \mathrm{Cd}$ internal-conversion source for energy calibration. Two Si surface-barrier detectors (5), mounted $180^{\circ}$ apart in azimuthal angle in a plane perpendicular to the electron polarization direction and at a scattering angle of $120^{\circ}$, detected electrons scattered from the target into a solid angle of $0.14 \mathrm{sr}$.

Since the entire Mott polarimeter was maintained at high voltage, the amplified detector outputs were converted to analog optical signals and transmitted to ground potential via Lucite light pipes, where they were converted back to electrical signals by photomultiplier tubes (PMT's) for processing by amplifiers and discriminators in preparation for counting by $10-\mathrm{MHz}$ scalers. A 512channel pulse-height analyzer was used to observe the energy spectrum of the detected electrons and to set each discriminator at a level that optimally rejected inelastic events. The pulse-height analyzer also served as a moni-

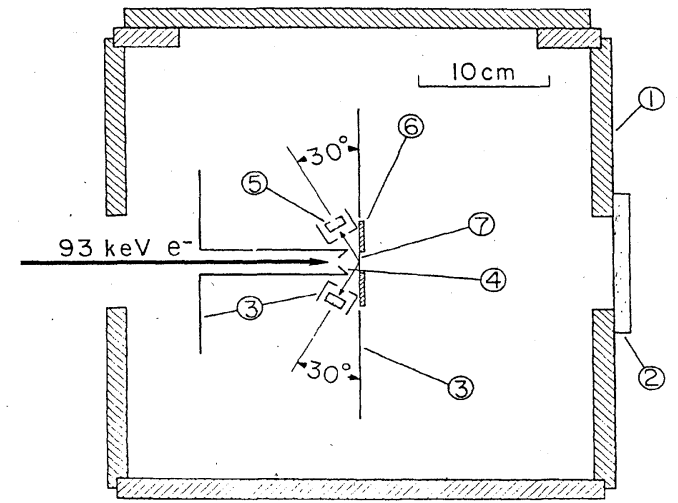

FIG. 4. Mott scattering region. The $93-\mathrm{keV}$ transversely polarized electrons enter from the left and are scattered by one of four gold targets in the target wheel, which can be rotated while the system is under vacuum and at high voltage. The following elements are indicated: (1) aluminum vacuum chamber; (2) Lucite window; (3) aluminum shielding, used to maximize the energy loss of electrons scattered from surfaces other than the gold target; (4) aluminum beam collimator; (5) surface-barrier detector (Ortec model SBEE100); (6) target wheel; (7) gold foil target.

tor of the electron beam energy which was referenced to the 62.5 - and $84.5-\mathrm{keV}$ internal conversion peaks of the ${ }^{109} \mathrm{Cd}$ source. In order to minimize the amount of elastic scattering occurring at surfaces other than the gold target, the components of the Mott chamber were constructed of aluminum and the target holder was coated with graphite.

\section{Hydrogen beam}

The atomic hydrogen beam apparatus is shown in Fig. 5. The main components included a tungsten oven (1) for thermal dissociation of $\mathrm{H}_{2}$, the hexapole magnet (8) for

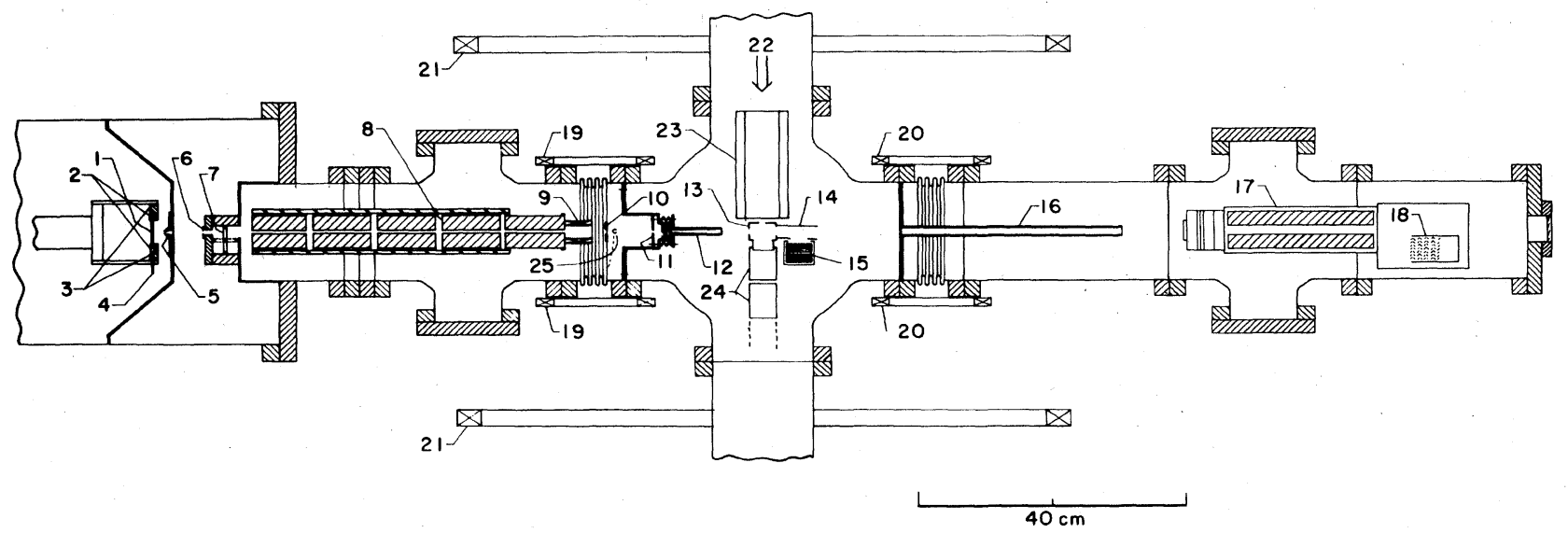

FIG. 5. Top-view scale drawing of the hydrogen-beam line showing the following elements: (1) tungsten oven; (2) molybdenum support blocks; (3) water-cooled terminals; (4) hydrogen gas inlet; (5) and (6) hydrogen-beam collimators; (7) butterfly valve; (8) hexapole magnet; (9) solenoid; (10) tuning fork beam-chopper (Bulova, type L40); (11) hydrogen-beam collimator; (12) tapered differential pumping tube; (13) interaction region, (14) electric field shield; (15) ion detector (Johnston Labs Model MM1-1S-FDB electron multiplier); (16) differential pumping tube; (17) quadrupole mass analyzer (Extranuclear Laboratories Model 270-9); (18) ion detector (EMI Model 9603/2B electron multiplier); (19) and (20) hydrogen atom spin rotation Helmholtz coils; (21) Helmholtz coils to define interaction region magnetic field (two orthogonal pairs not shown); (22) direction of incident electrons; (23) deceleration optics; (24) acceleration optics; (25) retractable Ar atomic beam source. 
state selection, appropriate magnetic fields [(9), (19), and (21)] following the hexapole to prevent Majorana depolarization, a tuning fork beam chopper (10), and, following intersection with the polarized electron beam, a quadrupole mass analyzer [(17) and (18)].

The hydrogen oven consisted of a $4.5 \times 4.9 \mathrm{~cm}^{2}$ section of $0.0025 \mathrm{~cm}$ tungsten foil rolled into a cylinder $4.5 \mathrm{~cm}$ long and $\sim 0.4 \mathrm{~cm}$ in diameter. The ends of this cylinder were mounted in molybdenum blocks (2), which in turn were attached to water-cooled copper blocks (3). Great care was taken to insure that one end of the oven could move in a direction parallel to the oven axis as freely as possible to relax the thermal stress on the tungsten. Hydrogen entered the cylinder at one end, and left by effusion through a hole $0.13 \mathrm{~cm}$ in diameter at the center. For optimizing the beam intensity, mechanical feedthroughs were incorporated to permit alignment of the hole while the oven was under vacuum and at its full operating temperature.

During the early measurements, the oven was heated resistively to $\sim 2800 \mathrm{~K}$ with a current of $140 \mathrm{~A}$ and $\mathrm{a}$ voltage of $5 \mathrm{~V}$. More recently, with the higher molecular hydrogen leak rate allowed by changes in the vacuum apparatus, an increased current of $180 \mathrm{~A}$ and voltage drop across the oven of $6 \mathrm{~V}$ was required to achieve a similar dissociation fraction. The effusing atoms and molecules were formed into a beam with the use of a skimmer (5) which preceded a differentially pumped region. In order to facilitate oven replacement, a butterfly valve (7) was used to isolate the hydrogen source and skimmer from the rest of the vacuum system.

The state-selection properties of hexapole magnets have been discussed by other authors, 5,111 and will not be rederived here. It suffices to say that ground-state hydrogen atoms with $m_{j}=+\frac{1}{2}$ are confined to a region near the axis of the hexapole and describe sinusoidal trajectories about the axis, while atoms with $m_{j}=-\frac{1}{2}$ are defocused. The hexapole magnet used in this work comprised five sections, each $8.3 \mathrm{~cm}$ long, with a 0.32 $\mathrm{cm}$-diam gap and a pole tip field strength of $8500 \mathrm{G}$. The total effective length of the magnet was $45.7 \mathrm{~cm}$, including 1.1-cm spacing between sections. Results of computer modeling of the focusing properties for this configuration indicated that the high-field atomic polarization was greater than 0.99 and was insensitive to small changes in the magnet and hydrogen-oven parameters. In regions of low magnetic field, such as the interaction region, coupling to the nuclear spin $I$ reduces the atomic polarization by a factor $1 /(2 I+1)$. However, because of the hyperfine coupling, the hexapole state selection itself is not independent of $m_{I}$, resulting in an atomic polarization at low field which is larger than the $1 /(2 I+1)$ factor. ${ }^{112}$ Based on these considerations, the atomic hydrogen polarization at low field was calculated to be $P_{\mathrm{H}}=0.50 \pm 0.02$.

The magnetic field in the hexapole is transverse and a function of azimuthal position; hence, the direction of the spins of the $m_{j}=+\frac{1}{2}$ atoms in the beam is not fixed, but rather is a function of the position of the atoms. In order to align the spins in one direction, a small solenoid (9) was placed at the exit of the hexapole. The 200-G longitudinal field of this solenoid insured that the spins of the atoms rotated adiabatically into one direction parallel to their momenta. The $\sim 5-G$ transverse field of a small Helmholtz pair (19) then produced adiabatic rotation of the spins into a direction either parallel or antiparallel to the electron-beam direction. In addition, three orthogonal pairs of Helmholtz coils (21) surrounding the interaction region chamber permitted a monotonic reduction of the magnitude of the field to $\sim 100 \mathrm{mG}$ while maintaining its direction either parallel or antiparallel to the electron beam. The magnitude and direction of the $\sim 100-\mathrm{mG}$ field were monitored frequently during operation.

At the exit of the small solenoid the hydrogen beam was modulated by a $100-\mathrm{Hz}$ tuning-fork beam-chopper (10) to permit subtraction of scattering events not related to the beam. Following the intersection with the electron beam, which occurred $\sim 90 \mathrm{~cm}$ from the hydrogen source, the hydrogen beam passed into a QMA that monitored its atomic and molecular composition. In order to facilitate tuning of the electron beam and the electron detector optics, a retractable room-temperature source of unpolarized argon atoms (25) was installed $\sim 22 \mathrm{~cm}$ upstream from

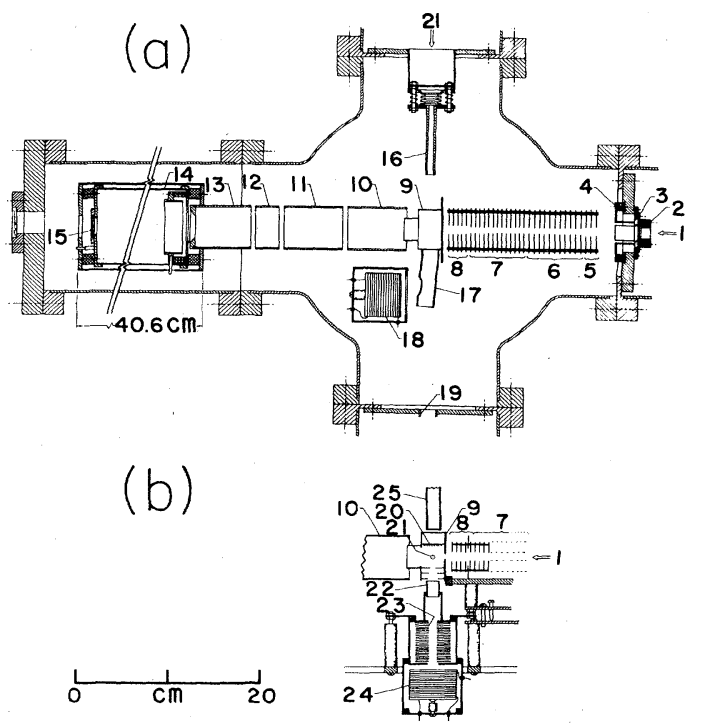

FIG. 6. Top-view scale drawing (a) of the interaction chamber and side-view scale drawing (b) of the interaction region looking upstream along the hydrogen beam with the following elements shown: (1) direction of incident electrons; (2) secondary electron repeller for electron current sensor (3); (4) electron-beam steering plates; (5) electron-beam focusing elements; (6) first deceleration filter lens; (7) second deceleration filter lens; (8) electron-beam focusing elements; (9) interaction region; (10)-(13) electron-beam reacceleration elements; (14) Faraday cup; (15) conducting-glass window for electron-beam alignment; (16) hydrogen-beam-line tapered differential pumping tube; (17) electric field shield; (18) ion detector (Johnston Labs Model MM1-1S-FDB electron multiplier); (19) hydrogenbeam-line differential pumping orifice; (20) CuBe baffle for suppression of surface-scattered electrons; (21) direction of incident hydrogen beam; (22) scattered-electron collector tube; (23) scattered-electron filter lens energy analyzer; (24) electron detector (Johnston Labs Model MM1-1S-FDB electron multiplier); (25) magnetic-field-probe vacuum insertion tube. 
the interaction region to enhance the scattering signal. In conjunction with the argon source, a movable beam flag was installed in the atomic beam line immediately before the interaction region. Under best conditions of effusive flow, the elastic counting rate was a factor of 10 larger for argon from this source than for atomic hydrogen from the oven. Argon was chosen since the $90^{\circ}$ elastic cross section at $20 \mathrm{eV}$ has been measured to be 3 times larger for $\mathrm{Ar}$ than for $\mathrm{H}_{2} \cdot{ }^{113-117}$

\section{Interaction region}

The elements of the interaction region are shown in Fig. 6. All of the components were compatible with the ultrahigh-vacuum requirements of $10^{-9}$ Torr and were capable of bakeout to $450^{\circ} \mathrm{C}$. The $1-\mathrm{keV}$ longitudinally polarized electrons (1) were incident from the right in the figure. Deceleration to $80 \mathrm{eV}$ was accomplished by onehalf of a hyperbolic potential filter lens (6), ${ }^{118}$ with a second, complete filter lens (7) used to decelerate the electrons to $\sim 8 \mathrm{eV}$ to trim the phase space. The second filter lens was also used to measure the energy width of the electron beam, as well as energy shifts with respect to the Fano source voltage. The same voltage supply which was used to bias the Fano source at $-1 \mathrm{keV}$ was also used to bias components in the interaction region, thus minimizing the effect of power supply drift. Further deceleration or acceleration to the desired beam energy was accom-

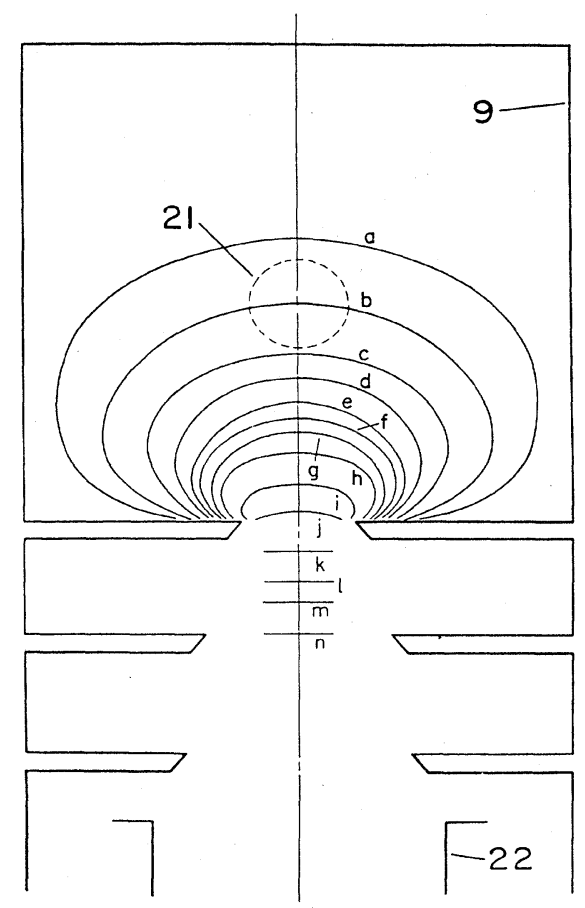

FIG. 7. Results of a two-dimensional resistive paper model of the interaction region, with the following elements shown: (9) interaction region; (21) cross section of hydrogen beam; (22) scattered-electron collector tube. For (22) biased at $+120 \mathrm{~V}$ with respect to (9), equipotential lines are [in volts relative to (9)] $a,+0.12 ; b, 0.18 ; c, 0.24 ; d, 0.30 ; e, 0.36 ; f, 0.42 ; g, 0.48 ; h$, $0.60 ; i, 0.84 ; j, 1.2 ; k, 2.4 ; l, 3.6 ; m, 4.8 ; n, 7.5$. plished by lens elements (8) and the potential on the interaction region box (9). After intersection with the hydrogen beam, the electrons were reaccelerated to $1 \mathrm{keV}$ [(10)-(13) in Fig. 6] and detected in a Faraday cup (14), as mentioned earlier. The hydrogen beam entered from the top in Fig. 6(a). Ions produced in the interaction region drifted a few millimeters downstream along the hydrogen beam and were accelerated into an electron multiplier (18).

Figure 6(b) shows the electron detector in detail. The hydrogen beam (21), with a diameter of $\sim 0.5 \mathrm{~cm}$, is viewed from downstream in the figure. Electrons scattered at $90^{\circ}$ were accelerated by the potential on cylindrical element (22) into a filter-lens energy discriminator (23) which transmitted only the elastically scattered electrons to an electron multiplier (24). With use of a twodimensional scale model which approximated the interaction region, it was determined with resistive paper that the typical bias on element $22[+120 \mathrm{~V}$ with respect to the interaction region (9)] changed the potential at the center of the interaction region by only $+0.2 \mathrm{~V}$, and changed the polar angle acceptance of the detection optics from a geometrical $\pm 12^{\circ}$ to $\pm 12.5^{\circ}$. Shown in Fig. 7 is an electric field map of the interaction region resulting from the resistive paper study.

\section{E. Timing and electronics}

Acquisition of electron-hydrogen scattering data was controlled by a Digital Equipment Corporation PDP/15 computer operating through a standard CAMAC (computer-aided measurement and control) interface with timing and data routing referenced to the $100-\mathrm{Hz}$ tuningfork hydrogen-beam chopper. Signals from both the electron and ion channels were routed to separate blind scalers according to whether the signals occurred during the 4msec hydrogen beam "on" portion or the 2-msec hydrogen-beam "off" portion of each 10-msec chopper cycle, as illustrated in Fig. 8. In addition, the QMA was switched between $\mathbf{H}$ and $\mathrm{H}_{2}$ every other chopper cycle; that is, every $20 \mathrm{msec}$, by a square-wave pulse train ( $Q$ in Fig. 8) at the QMA sweep input. For atomic and molecular components both QMA beam-on and beam-off signals were routed to separate blind scalars. [Only the second beam-on portion of the $\mathbf{H}$ and $\mathbf{H}_{2}$ double cycles was acceptable as QMA signal, since the settling time (time required for the QMA to reach stable operation) after switching the mass value was $2 \mathrm{msec}$. The effect of the settling time can be seen in Fig. 8 as longer QMA preamp zero levels each time the $Q$ signal is stepped.] Also recorded in blind scalers were the number of chopper cycles and the digitized Faraday-cup current.

Since counting rates in the $90^{\circ}$ elastic channel were low ( $1-5$ events per s), a large effort was made to reduce background events. As expected, proper tuning of the electron beam was essential for the reduction of wall- and aperture-scattered events. However, even under optimal conditions, extraneous pulses persisted, particularly during the first few days after ultrahigh vacuum was achieved. These "noise" pulses, thought to have been microdischarges in the electron detector caused by mercury, 
HYDROGEN BEAM TIMING

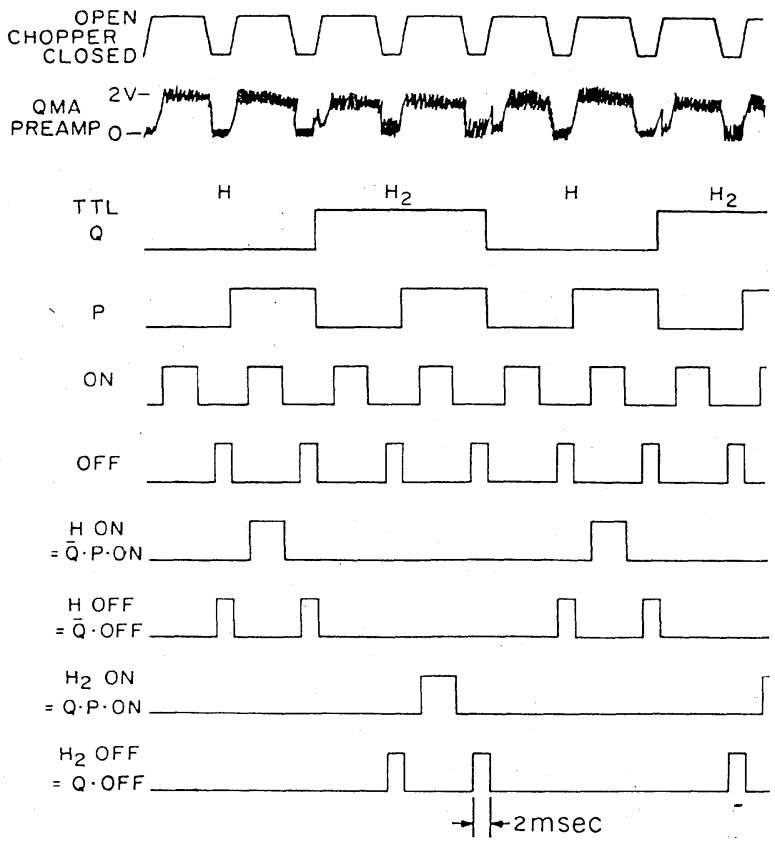

FIG. 8. Schematic diagram of timing and signal gating relative to the chopped hydrogen beam. All signals were synchronized with the $100-\mathrm{Hz}$ tuning-fork chopper electronics. The onset and width of the OFF gate was set to correspond to the QMA low output. The width of the ON gate was twice that of the OFF gate, while its position was centered with respect to the QMA high output. The $P$ and $Q$ pulses were derived from the OFF gate, with the $P$ level changing at the end of every OFF pulse and the $Q$ level changing at the end of every other OFF pulse. The $Q$ pulse train was applied to the QMA sweep input to switch detection of the QMA between $\mathrm{H}$ and $\mathrm{H}_{2}$. The acquisition of the four QMA signals, $\mathrm{H}$ on, $\mathrm{H}$ off, $\mathrm{H}_{2}$ on, and $\mathrm{H}_{2}$ off, was determined by the corresponding gates derived as shown.

were significantly longer than "true" pulses, as shown at the bottom of Fig. 9. This characteristic was used effectively to discriminate against them, as any pulse which remained within the window of the single-channel analyzer (SCA) but which did not cross zero within $2 \mu \mathrm{sec}$ was discarded. Figure 9 shows the timing logic, and Fig. 10 presents a block diagram of this discrimination circuit.

In the ion channel, because of the higher counting rates, (12-228 events per s), detector and electronic noise essentially posed no problems. However, a few percent of the bipolar pulses produced by the amplifier displayed saturation effects characterized by a positive excursion on the return to the baseline, thus causing the SCA to fire and to register a spurious second event. In order to prevent double counting, a $10-\mu \mathrm{sec}$ delay was introduced into the SCA and lower-level discriminator (LLD) signals, as shown in Fig. 11. In this manner the detection of the overshoot, which typically occurred $6 \mu \mathrm{sec}$ after the initial pulse, was precluded.

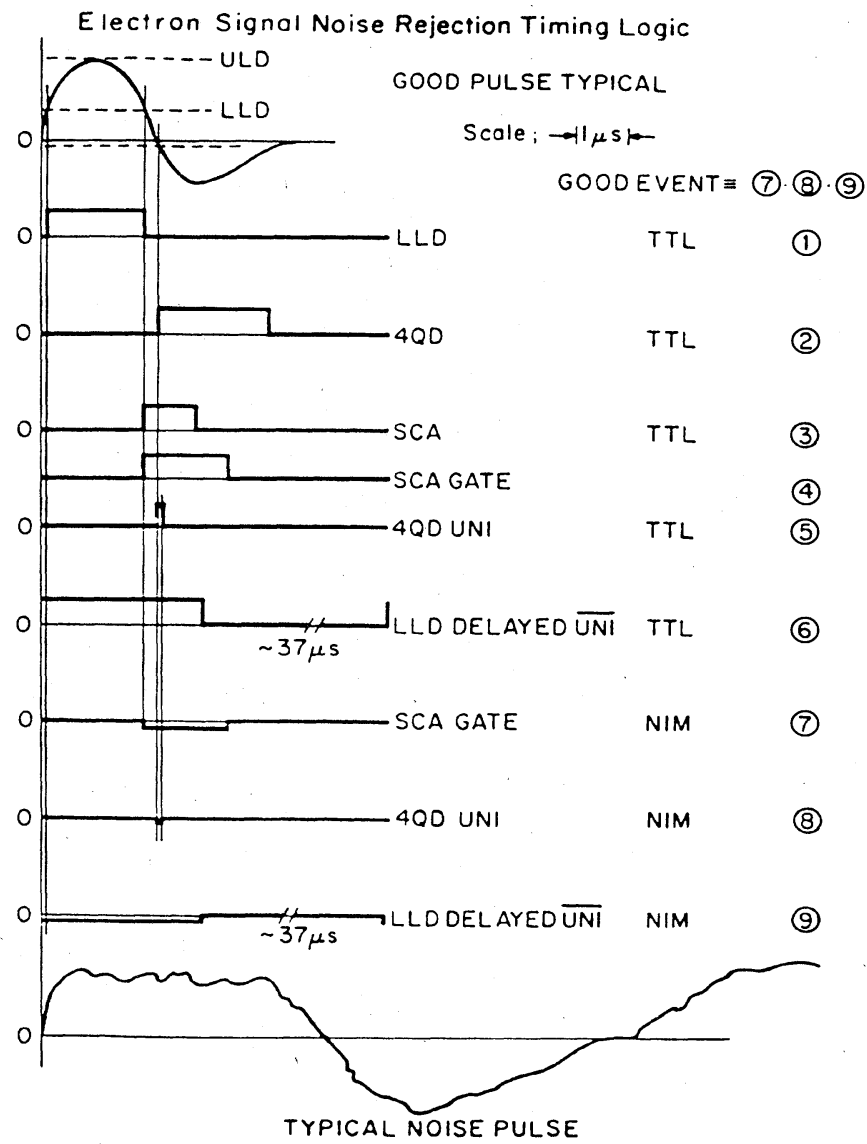

FIG. 9. Schematic diagram of the timing and signal gating to reject noise in the electron signal. LLD and ULD refer to the lower- and upper-level discriminators, respectively, of the singlechannel analyzer (SCA). The 4QD is a four-quadrant discriminator which could fire a TTL pulse when the input passed a preset level $(-10$ to $+10 \mathrm{~V})$ with the correct slope $(+$ or -$)$. It was set to fire when the amplifier output went negative. GATE, UNI, and DELAYED $\overline{\text { UNI }}$ refer to pulses generated by delay gate generators and associated one-shot univibrators.

Electron Signal Noise Rejection Logic

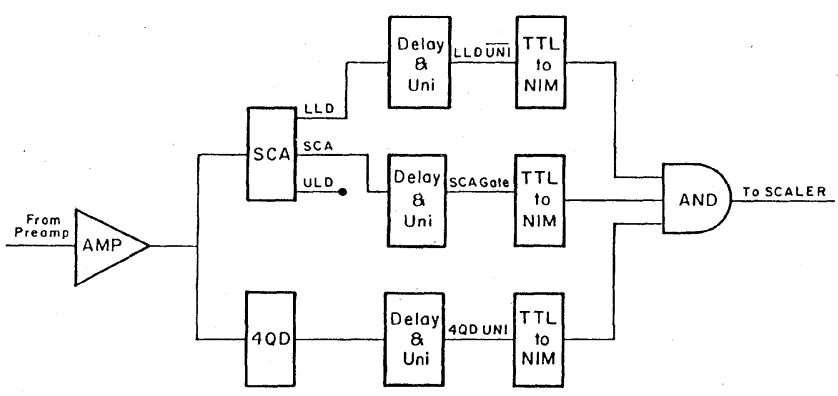

FIG. 10. Block diagram of the electron signal noise rejection circuitry. Components included Ortec Model 109A preamplifier, Canberra Model $818 \mathrm{Amp} / \mathrm{SCA}, 4 \mathrm{QD}$ four-quadrant discriminator build at Yale, Yale EPI model 121 dual delay and univibrator gate generator, standard TTL to NIM three-fold logic unit used as a three-input AND gate. 


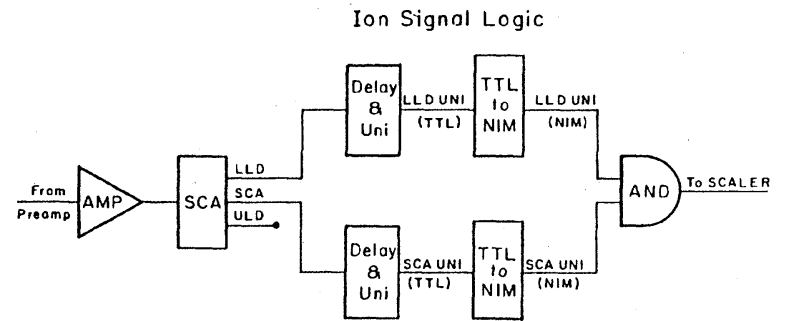

FIG. 11. Block diagram of the ion signal circuitry used to prevent double counting.

\section{EXPERIMENTAL PROCEDURE}

\section{A. Data acquisition for $e^{-}-\mathrm{H}$ scattering}

The first experiments that we performed provided information about $A_{I}$ alone. In addition, early exploratory measurements of $A_{90^{\circ}}(1 S \rightarrow 1 S)$ were carried out separate1y. The discussion in this section applies to the method we adopted in our recent work which permitted the concurrent measurement of $A_{90^{\circ}}(1 S \rightarrow 1 S)$ and $A_{I}$. The general experimental method, however, is substantially the same for both the early and the recent work.

For each charge of cesium in the Fano source, an electron polarization measurement was made prior to the acquisition of electron-hydrogen scattering data. The polarized electrons were then transported to the hydrogen interaction region, their approximate energy being determined by the voltage applied to the interaction region relative to the source. The absolute energy as well as the energy spread of the electrons were obtained from measurement of the threshold for ionization and from filter-lens scans, as discussed in Sec. III C. Following these measurements, acquisition of electron-hydrogen collision data was initiated with the use of the PDP/15 computer.

In order to reduce systematic effects associated both with polarization reversal and with possible timedependent variations of experimental parameters, frequent reversal of the electron polarization and of the atomic hydrogen polarization was required for the measurement of the spin-dependent cross-section asymmetries. To this end, the electron polarization was reversed every 5-10 s by $90^{\circ}$ rotation of the Fano-source quarter-wave plate. Further modulation of the electron polarization was accomplished by $90^{\circ}$ rotation of the Fano-source linear polarizer every 20-40 min. As a final precaution, the atomic hydrogen polarization was reversed every $4-8 \mathrm{~h}$ by the reversal in direction of the $\sim 100-\mathrm{mG}$ magnetic field in the interaction region.

For each quarter-wave-plate position, $e^{-}-\mathrm{H}$ scattering data were accumulated until a preset charge had entered the Faraday cup. The computer then halted data acquisition, read and cleared the blind scalers, advanced the quarter-wave plate by $90^{\circ}$, and reinitiated data accumulation, having stored the following information:

(1) ion events with $\mathrm{H}$ beam on,

(2) ion events with $\mathrm{H}$ beam off,

(3) electron events with $\mathbf{H}$ beam on,
(4) electron events with $\mathbf{H}$ beam off,

(5) difference in QMA H signal with beam on and off,

(6) difference in QMA $\mathrm{H}_{2}$ signal with beam on and off,

(7) digitized Faraday-cup current, and

(8) elapsed time.

During data acquisition, the accumulated totals for each of the four quarter-wave-plate positions were displayed on a storage scope. As an alternate display, the histograms of each of the eight data channels were shown as a function of time to permit a careful review of beam stability and possible detector noise. After approximately 40 complete rotations of the quarter-wave plate, the run was halted, the data were written onto magnetic tape for off-line analysis, the Fano-source linear polarizer was manually rotated $90^{\circ}$, and a new run was started. At the completion of four runs, each corresponding to one of the four linear polarizer positions, the hydrogen-oven temperature was lowered from $\sim 2800$ to $\sim 1400 \mathrm{~K}$, and a run was made to determine the fraction of events attributable to scattering from $\mathrm{H}_{2}$, as discussed in Sec. III D and Appendix A. The oven temperature was then returned to $\sim 2800 \mathrm{~K}$, a filter-lens scan was taken to check for any shift in electron energy, and another set of four runs was begun. At the end of the nine runs, the direction of the $\sim 100$-mG magnetic field was reversed, another filter-lens scan was taken, and the sequence of nine runs repeated. A complete measurement at one incident electron energy typically consisted of 16 hot-oven-temperature runs and 2 cold-oven-temperature runs, corresponding to two complete rotations of the linear polarizer for each of the two atomic hydrogen polarization directions. Approximately 300-1500 elastic events were recorded for each hot run, the number depending principally on the energy, intensity, and focusing of the polarized electrons in the interaction region. For an electron current of $2 \mathrm{nA}$ in the interaction region, an atomic hydrogen density of $\sim 10^{9} / \mathrm{cm}^{3}$ (as inferred from scattering rates), and a background gas pressure of $10^{-9}$ Torr, the elastic scattering count rate ranged from 1 to $5 \mathrm{~s}^{-1}$, with signal-to-background ratios ranging from 1 to 4.5. The ionization count rate obtained under the same conditions ranged from 12 to $228 \mathrm{~s}^{-1}$, with values of signal-to-background ratios ranging from 1.7 to 15.5. These rates were the observed $H$-beam-on event rates, which were reduced by the 0.4 duty factor of the hydrogen beam.

Upon the completion of a measurement at one energy, the amount of unused Cs was estimated and the condition of the hydrogen oven assessed. If conditions were favorable, a measurement at another energy was begun. The 60-g Cs load produced polarized electrons of the required intensity for $\sim 40 \mathrm{~h}$, and a good hydrogen oven had a lifetime of $\sim 25 \mathrm{~h}$ at operating temperature. Usually, an additional Mott measurement of the electron polarization was completed near the end of the 40 -h cesium load lifetime.

For both elastic scattering and impact ionization, the quantity measured for each run was the real asymmetry $\Delta_{R}$ defined as

$\Delta_{R}= \pm\left(N_{02}-N_{13}-B_{02}+B_{13}\right) /\left(N_{02}+N_{13}-B_{02}-B_{13}\right)$, 
where $N_{02}$ is the sum of H-beam-on events for quarterwave-plate positions 0 and $2\left(0^{\circ}\right.$ and $\left.180^{\circ}\right), N_{13}$ is the equivalent sum for quarter-wave-plate positions 1 and 3 $\left(90^{\circ}\right.$ and $\left.270^{\circ}\right)$, and $B_{02}$ and $B_{13}$ are twice the corresponding $\mathrm{H}$-beam-off sums, since the $\mathrm{ON}$ gate was twice as long as the OFF gate (see Sec. II E). The positive (negative) sign in Eq. (22) applies when the direction of the interaction region magnetic field is such that $N_{02}$ corresponds to electron and atomic spins antiparallel (parallel). For each process, the experimental asymmetry $\Delta_{R}$ is related to the corresponding physical asymmetry $\boldsymbol{A}$, defined in Eqs. (2) and (9), by

$$
\Delta_{R}=P_{e} P_{\mathrm{H}}\left(1-F_{2}\right)|\cos \alpha| A,
$$

where $P_{e}$ and $P_{\mathrm{H}}$ are the electron and atomic hydrogen polarizations, respectively, $F_{2}$ is the fraction of events attributed to scattering from molecular hydrogen, and $\alpha$ is the angle between the electron beam and the interaction region magnetic field. Frequent monitoring of the magnetic field insured that $|\cos \alpha|$ remained greater than 0.985 .

In addition to the real asymmetry $\Delta_{R}$, two false asymmetries $\Delta_{F 1}$ and $\Delta_{F 2}$ were calculated, corresponding to different combinations of quarter-wave-plate positions, combination $0+1-2-3$ and combination $0+3-1-2$, respectively. These false asymmetries, formed from the difference of the sums of events with an equal number of incident positive and negative helicity electrons, should be zero in the absence of systematic effects arising from either electron polarization reversal or other time-dependent experimental parameters. A discussion of the data analysis involving these asymmetries will be presented in Sec. IV.

\section{B. Electron polarization measurement}

The Mott scattering apparatus described in Sec. II B was used to measure the electron polarization. Unpolarized electrons, produced by photoionization of cesium vapor with the linear polarizer removed from the Fano optical train, were used to tune the beam to the Mott target and to set the two detectors to approximately equal counting rates. With the linear polarizer in place, the alignment of the beam was fine-tuned to reduce to a negligible value the instrumental asymmetry produced by beam misalignment. Data from the two silicon surface-barrier detectors were then collected for each of the four quarterwave-plate positions for typically $15 \mathrm{~s}$, with $\sim 40000$ events recorded for the sum of the two detectors. After one complete rotation of the quarter-wave plate, a gold target of a different thickness was moved into the beam position, and data were again collected for each of the quarter-wave-plate positions. The entire procedure was then repeated for each of the three other linear polarizer positions. A complete Mott polarization measurement comprising four different gold targets required no more than $30 \mathrm{~min}$.

A typical pulse-height spectrum from one of the two detectors is shown in Fig. 12. The $13-\mathrm{keV}$ energy width of the elastic peak is attributable to the resolution of the detectors, a contention borne out by the presence of the

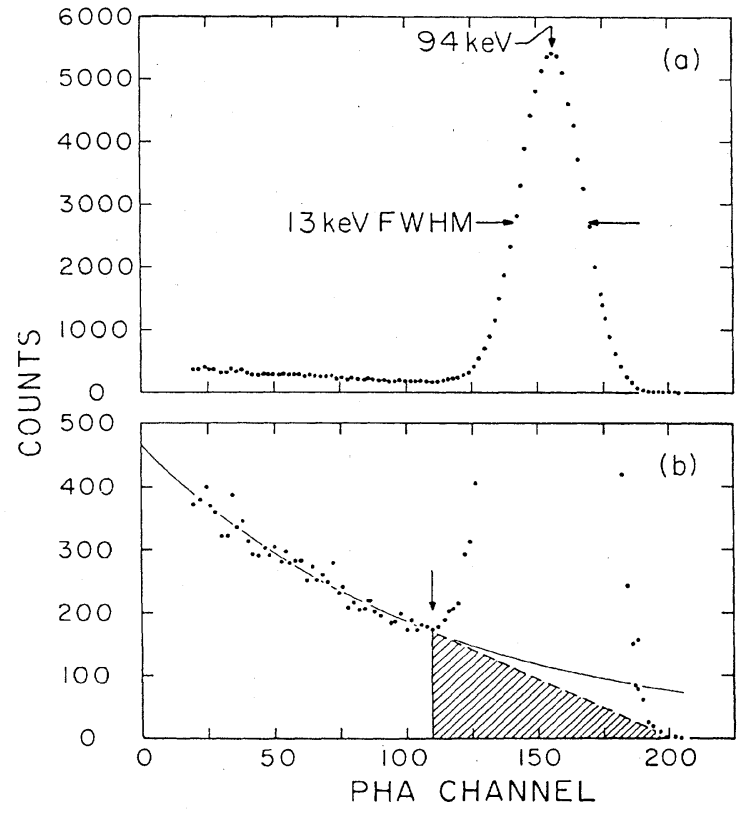

FIG. 12. Mott scattering pulse-height-analysis spectrum. The vertical scale is a factor of 10 lower in (b) than in (a). The shaded area represents the inelastic background subtraction, and the arrow indicates the discriminator threshold. The solid line gives the exponential fit to the inelastic scattering below threshold.

same width for the spectra of internal conversion electrons from the ${ }^{109} \mathrm{Cd}$ source. For each detector, a discriminator was set at the "knee" of the Mott spectrum with pulses above the discriminator threshold recorded in a $10-\mathrm{MHz}$ scaler. In order to avoid nonlinearities in the PMT's, however, counting rates were always maintained below $2000 / \mathrm{s}$ by the insertion of a perforated screen in the uv light beam of the Fano source.

The measured Mott asymmetry $\Delta_{M}$ is defined by

$$
\Delta_{M}=\frac{1-\xi}{1+\xi},
$$

where

$$
\xi=\left(\frac{N_{1}^{+} N_{2}^{-}}{N_{2}^{+} N_{1}^{-}}\right)^{1 / 2}
$$

with $\left(N_{i}^{ \pm}\right)$being the number of electrons scattered from a gold target into detector $i(i=1,2)$ for positive-helicity $(+)$ and negative-helicity $(-)$ light, corrected for background effects including inelastic scattering from the target and the chamber walls, backscattering from the detectors, and elastic and inelastic scattering from the Formvar backing. ${ }^{119}$ With the electron beam blocked, detector and electronic noise above the discriminator threshold was found to be less than $0.1 \%$ of the Mott counting rate and hence was neglected. Also negligibly small $(<0.2 \%$ of the total event rate) at the low beam current used was the pile-up peak in the pulse-height spectrum at twice the energy of the incident beam.

From the measurement of $\Delta_{M}$ for four gold target 
thicknesses, ranging from 27 to $62 \mu \mathrm{g} / \mathrm{cm}^{2}$, effects of plural and multiple scattering on the Mott asymmetry were quantified. Various extrapolations to effective zero target thickness were investigated and the weighted average of the extrapolated values of $\Delta_{M}$ was determined. A detailed explanation of the procedures used, the justification for these procedures, and some other insights into systematic effects on Mott scattering are contained in a separate publication. ${ }^{108}$ For the thickest target the measured depolarization was $18 \%$, while for the thinnest target the depolarization was $7 \%$.

The polarization $P_{e}$ of electrons from the Fano source was determined from the relation

$$
P_{e}=\Delta_{M}(t=0) / S(\theta),
$$

where $\Delta_{M}(t=0)$ is the Mott asymmetry extrapolated to effective zero target thickness and the Sherman function $S(\theta)$ for the kinematics used is $-0.387 \pm 0.008$, as interpolated from published calculations. ${ }^{120,121}$ The $2 \%$ uncertainty in $S(\theta)$ was added linearly to the $3 \%$ uncertainty ${ }^{108}$ in the extrapolated value $\Delta_{M}(t=0)$, resulting in a $5 \%$ uncertainty in $P_{e}$. During the course of the experiment, $P_{e}$ varied from 0.42 to 0.75 , the value at any particular time depending principally on the degradation of the dichroic linear polarizer ${ }^{122}$ in the Fano source optical train.

\section{Electron energy measurement}

The possibility of shifts in the polarized electron energy, caused by cesium deposition on the Fano-source elec-
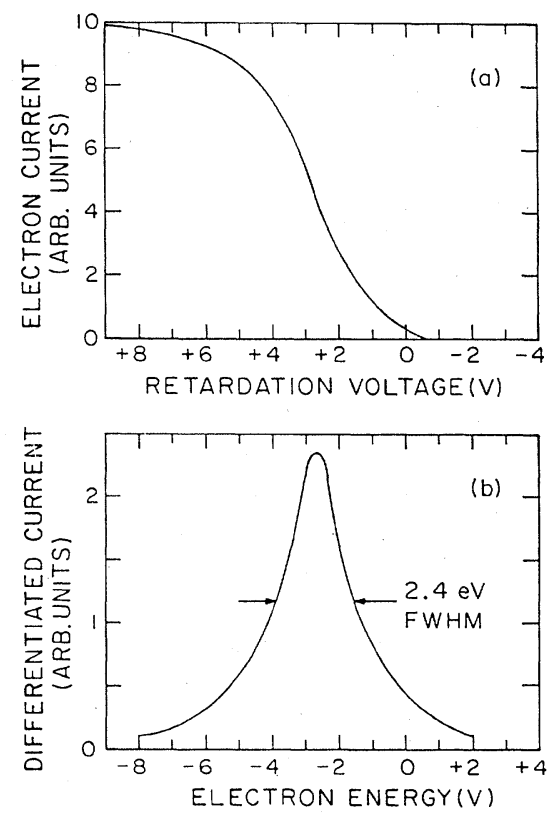

FIG. 13. Filter-lens energy profile of the polarized electron beam. (a) Electron current transmitted as a function of the retardation voltage measured relative to the $-1-\mathrm{kV}$ potential of the Fano source. (b) Differentiated form of (a), revealing the polarized electron energy spread, with electron energy given relative to $1 \mathrm{keV}$. trodes with consequent work-function shifts, necessitated frequent measurements of the electron energy. The relative energy and energy width of the polarized electron beam were determined by measurements of the current transmitted by the filter-lens energy analyzer as a function of its retardation potential. ${ }^{118}$ Such a transmission curve is shown in Fig. 13(a). The derivative of this curve gives the energy profile of the polarized electron beam and is shown in Fig. 13(b). In the recent studies ${ }^{23,24}$ the FWHM energy spread of the polarized electrons varied from 2.3 to $2.7 \mathrm{eV}$ with a typical value of $2.5 \mathrm{eV}$, as contrasted with $3.0 \mathrm{eV}$ in the early studies. ${ }^{16-18}$ The difference is attributed to a reduction in the size of the photoionization region in the Fano source caused by the insertion of the Freon-cooled aperture.

During data acquisition, a filter-lens energy scan was performed every three to four hours. Since any shifts in electron energy due to work-function fluctuations in the source were indicated by identical energy shifts in the filter-lens scans, these scans provided an easy method for monitoring the energy. Typically, shifts in energy were less than $0.2 \mathrm{eV}$ per $10 \mathrm{~h}$ of operation.

The relative energy measurements obtained with the filter lens were placed on an absolute scale by measurements of impact ionization of atomic hydrogen in the energy region near threshold using unpolarized electrons. The ionization signal as a function of energy near threshold is shown in Fig. 14. In conformity with McGowan and Clark's ${ }^{97}$ determination that the ionization cross section varies linearly with energy from $\sim 0.4$ to $\sim 3.0 \mathrm{eV}$ above threshold, the present data were fit to a line with the voltage intercept interpreted as the ionization threshold of $13.6 \mathrm{eV}$. The uncertainty in the intercept (and hence in the absolute energy scale) of $\pm 0.2 \mathrm{eV}$ is small compared to the energy width. A filter-lens scan of the unpolarized electron beam used in the threshold study then served to calibrate the filter lens on an absolute energy scale. The energy profile of the unpolarized electrons is shown in Fig. 15.

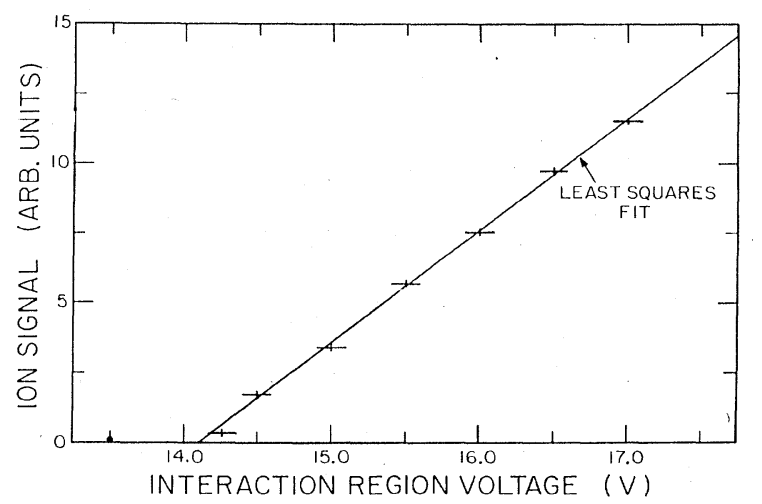

FIG. 14. Ionization threshold energy calibration. These measurements of the ion signal as a function of the potential of the interaction region were made with unpolarized electrons. The horizontal bars represent the estimated $\pm 0.1-\mathrm{eV}$ relative uncertainty in the electron energy. 


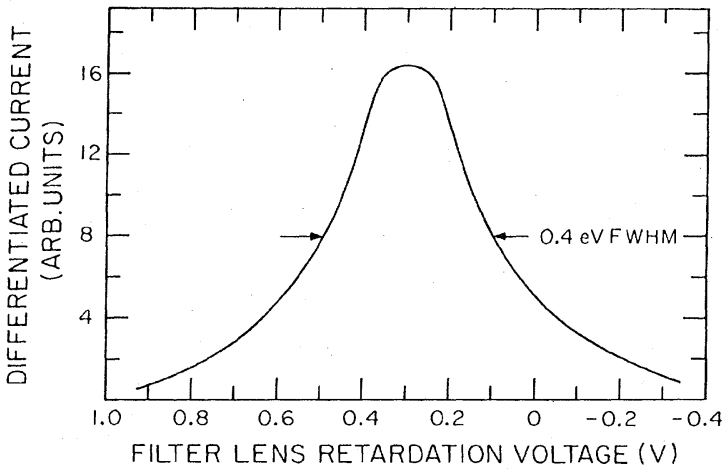

FIG. 15. Filter-lens energy profile of the unpolarized electron beam. This graph is similar to Fig. 13(b) for polarized electrons. The energy spread of $0.4 \mathrm{eV}$ FWHM is attributed mainly to the resolution of the filter lens, which was calculated to be $0.35 \mathrm{~V}$ (Ref. 118).

\section{Determination of molecular fraction}

The fraction of events originating from collisions with molecular hydrogen was determined by continuously monitoring the atomic and molecular composition of the beam and periodically measuring the scattering events at a lower hydrogen-oven temperature for which the beam was entirely molecular. Both the elastic events and the impact ionization events were treated by a similar analysis.

Let

$$
N^{\prime}(T)=[N(T)-B(T)] / I_{e}
$$

represent the number of scattering events, corrected for background and normalized to the incident electron current $I_{e}$, that are obtained at a hydrogen-oven temperature $T$. Then if $N_{1}^{\prime}(T)$ and $N_{2}^{\prime}(T)$ are the corresponding number of events due to atoms and molecules, respectively, $N^{\prime}(T)$ is given by

$$
N^{\prime}(T)=N_{1}^{\prime}(T)+N_{2}^{\prime}(T),
$$

in which case the fraction $F_{2}(T)$ of events attributed to molecules alone becomes

$$
F_{2}(T)=N_{2}^{\prime}(T) / N^{\prime}(T) .
$$

For "cold" temperatures, $T=T_{c} \sim 1400 \mathrm{~K}$, the beam, as inferred from thermodynamic considerations, ${ }^{123}$ consisted entirely of $\mathrm{H}_{2}$, and the ratio

$$
\Lambda(T)=N^{\prime}(T) / Q_{2}(T)
$$

of the number of scattering events to the quadruplemass-analyzer $\mathrm{H}_{2}$ signal rate $Q_{2}(T)$ becomes

$$
\Lambda\left(T_{c}\right)=N^{\prime}\left(T_{c}\right) / Q_{2}\left(T_{c}\right)=N_{2}^{\prime}\left(T_{c}\right) / Q_{2}\left(T_{c}\right) .
$$

Because the collision process and the mass-analyzer ionization process both depend only on the beam density, and because there is no velocity-dependent focusing of $\mathrm{H}_{2}$ by the hexapole magnet, the ratio $N_{2}^{\prime}(T) / Q_{2}(T)$ is assumed to be independent of temperature. The measurement of $\Lambda\left(T_{c}\right)$ reflects the sensitivity of the collision to molecular contamination. In terms of $\Lambda$, the fraction $F_{2}$ can be rewritten as

$$
F_{2}(T)=\frac{N_{2}^{\prime}(T)}{Q_{2}(T)} \frac{Q_{2}(T)}{N^{\prime}(T)}=\frac{\Lambda\left(T_{c}\right)}{\Lambda(T)} .
$$

In addition it can be shown (see Appendix A) that for conditions of constant beam geometry and constant QMA, electron, and ion detector acceptances and efficiencies, $\Lambda(T)$ varies linearly with $\Gamma(T)$, where $\Gamma(T)$ is defined as

$$
\Gamma(T) \equiv Q_{1}(T) / Q_{2}(T),
$$

the ratio of QMA atomic to molecular signals.

Plots of $\Lambda(T)$ versus $\Gamma(T)$ were made for both magnetic field orientations at each incident electron energy for both elastic scattering and impact ionization. The linear relationship between $\Lambda^{e}(T)$ (for elastic scattering) and $\Gamma(T)$ is illustrated in Fig. 16 for incident $6.3-\mathrm{eV}$ polarized electrons. Since $\Gamma(T)$ could be determined with much higher precision than $\Lambda^{e}(T)$ during each run, a linear leastsquares fit was applied to the data, with the $\Lambda^{e}(\Gamma(T))$ values from the fit being used in Eq. (32) to obtain $F_{2}^{e}$. Values of $F_{2}^{e}$ ranged from 0.05 to $0.20( \pm 10 \%)$ with the consequence that the fraction of elastic events arising from atomic hydrogen, $\left(1-F_{2}^{e}\right)$, ranged from 0.80 to 0.95 $( \pm 2 \%)$.

A similar analysis was performed to determine $F_{2}^{I}$, the fraction of detected ion events produced by collisions with $\mathrm{H}_{2}$. However, it was found that for the recent ionization measurements the dependence of $\Lambda^{I}(T)$ on $\Gamma(T)$ became nonlinear for oven temperatures $T \leq 1600 \mathrm{~K}$, as can be seen in Fig. 17. This nonlinear behavior, which was not seen in the early ionization measurements, ${ }^{16}$ nor in the recent elastic measurements ${ }^{23}$ (see Figs. 16 and 18), is attri-

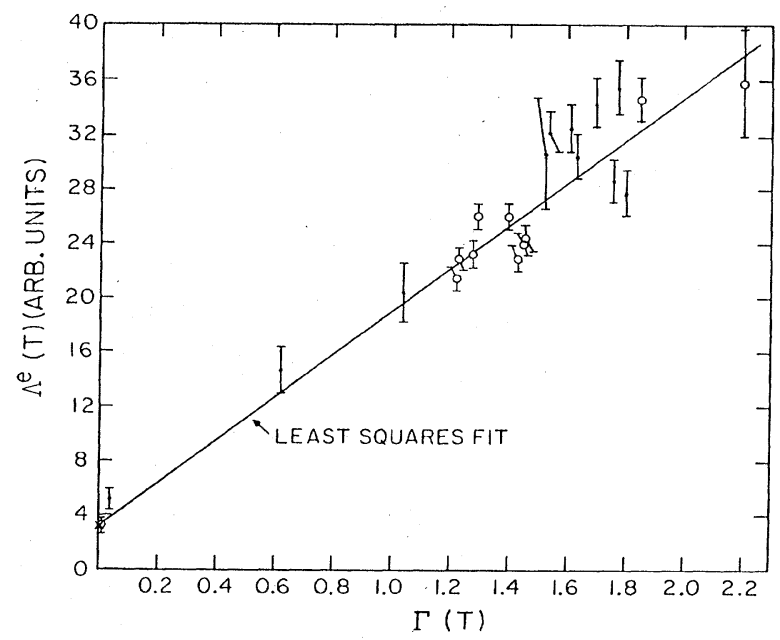

FIG. 16. Measurements of $\Lambda^{e}(T)$, the ratio of elastic scattering events to QMA $\mathrm{H}_{2}$ signal, as a function of $\Gamma(T)$, the ratio of QMA $\mathrm{H}$ and $\mathrm{H}_{2}$ signals, for 6.3-eV polarized electrons. Solid points are for the hydrogen polarization direction along the direction of incident electron momenta. Open circles are for the hydrogen polarization direction opposite to the direction of incident electron momenta. 


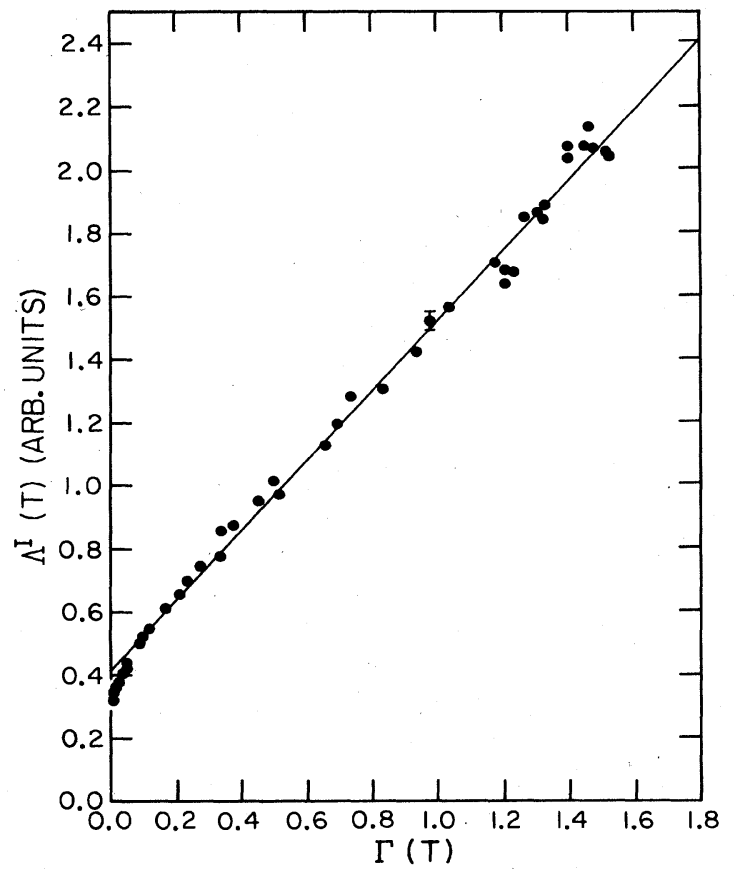

FIG. 17. Measurements of $\Lambda^{I}(T)$, the ratio of ionization events to QMA $\mathrm{H}_{2}$ signal, as a function of $\Gamma(T)$ for $22.2 \mathrm{-eV}$ polarized electrons. Data from both hydrogen polarization directions are included. The nonlinear behavior for small $\Gamma(T)$ $(T \lesssim 1600 \mathrm{~K})$ is attributed to incomplete ion detector acceptance.

buted to changes in the detector acceptance for ions produced from the slow $\mathrm{H}_{2}$ in the lower temperature beams. The recoil angles of the $\mathrm{H}_{2}{ }^{+}$ions increase as the beam velocity decreases. The probable cause of the reduced acceptance for larger-recoil-angle ions is a small decrease in the angle of the electric field shield [(17) of Fig. 6(a)] made prior to these measurements.

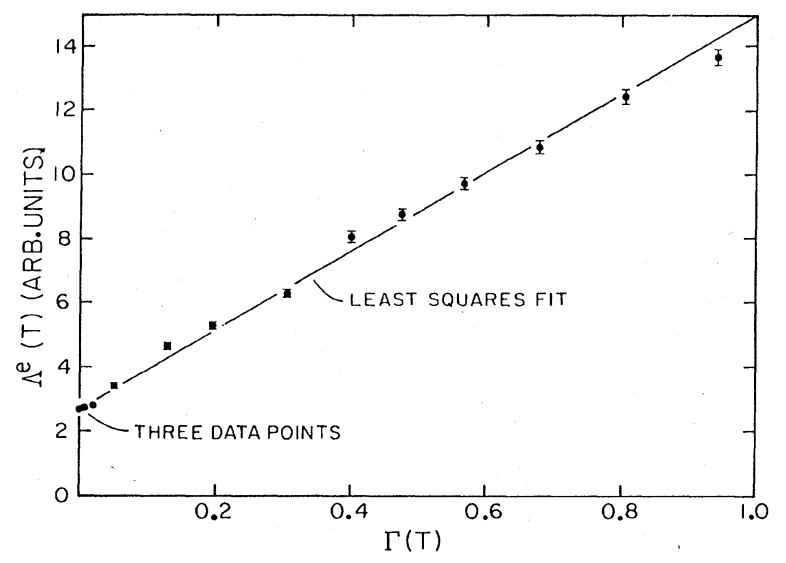

FIG. 18. Measurements of $\Lambda^{e}(T)$ as a function of $\Gamma(T)$ for 23.2-eV unpolarized electrons. The nonlinear behavior which appeared in the ionization data (Fig. 17) is not present in the elastic scattering data. The measurements with polarized electrons shown in Fig. 16 were repeated here with unpolarized electrons to improve the statistics.
In order to determine $F_{2}^{I}, \Lambda^{I}(T)$ and $\Gamma(T)$ were measured at each incident energy for several values of the oven temperature $T$ from 1200 to $2800 \mathrm{~K}$. For $T \geq 1600$ $\mathrm{K}$ the dependence of $\Lambda^{I}(T)$ on $\Gamma(T)$ was linear and these data were fit to a straight line, an extrapolation of which provided the true value $\Lambda^{I}\left(\Gamma\left(T_{c}\right)\right)$, where the temperature $T_{c}$ was defined by an input power to the oven of $\sim 130 \mathrm{~W}$ and was approximately $1400 \mathrm{~K}$. The extrapolated value $\Lambda^{I}\left(\Gamma\left(T_{c}\right)\right)$ was then used in the numerator of Eq. (32) to obtain $F_{2}^{I}$. In general, allowance for the nonlinear behavior of $\Lambda^{I}$ resulted in a 4-5\% decrease in the values of $\left(1-F_{2}^{I}\right)$, with a corresponding increase in the values of $A_{I}{ }^{24}$

The determination of $F_{2}^{I}$ was also affected by the presence of an asymmetry

$$
\Delta_{B} \equiv \pm\left(B_{02}-B_{13}\right) /\left(B_{02}+B_{13}\right)
$$

in the background ion events accumulated at the hot oven temperature $(T \sim 2800 \mathrm{~K})$ during the hydrogen-beam-off portion of the beam-chopper cycle. This background asymmetry is attributed to the velocity spread of the hydrogen beam and the constraints imposed by the OFF gate in the data-acquisition sequence, which resulted in some hydrogen-beam-related events occurring during the OFF time. The magnitude of $\Delta_{B}$ is of the order $\Delta_{R} / 4$ and has the same sign as $\Delta_{R}$. That such an effect is not observed in the elastic scattering data appears to be due to the larger statistical uncertainties associated with the elastic measurements. The presence of such a background asymmetry does not affect the determination of $\Delta_{R}$ but does influence the determination of $F_{2}^{I}$ and hence $A_{I}$ itself, as is described in Appendix B. The resulting increase in the uncertainties in $\left(1-F_{2}^{I}\right)$ typically was less than $1 \%$ of the value of $\left(1-F_{2}^{I}\right)$, producing a $\sim 5 \%$ increase in the $A_{I}$ uncertainties for both the early and recent data. Values of $F_{2}^{I}$ varied from $0.02 \pm 0.01$ to $0.45 \pm 0.09$ with a typical value of $0.16 \pm 0.03$.

\section{DATA ANALYSIS}

\section{A. General review of data}

The analysis of the elastic scattering and impact ionization data began with a careful search of the information stored on magnetic tape for evidence of nonstatistical behavior. For each run the histograms of both the ion signal and electron signal with hydrogen beam on and off were examined. If detector or electronic noise was found in any of these four data channels corresponding to a particular quarter-wave-plate data bin, the data in the bin were discarded, and the data in the corresponding bin for the other seven channels were automatically discarded as well. The stability of both the electron beam and the atomic beam was also checked from the histograms of Faraday-cup current and QMA $\mathrm{H}$ and $\mathrm{H}_{2}$ signals. Occasionally during a run, the hydrogen-oven temperature changed, producing a small but abrupt change in the QMA atomic and molecular signals; under these conditions, the run was divided into two runs, each corresponding to a different oven temperature. In no case were any systematic effects on the asymmetries found that were due 
to run-to-run variations in the beam intensities or in the signal-to-noise ratios.

Once the data had been reviewed for the obvious systematic effects, $\Delta_{R} /\left(1-F_{2}\right)$ was calculated with its statistical uncertainty for each run for both elastic and ionization events. A $\chi^{2}$ analysis was then performed on these results for each target polarization individually and for both groups together. [For incident energies of 27.0 and $30.3 \mathrm{eV}$ the analysis was performed on the quantity $\Delta_{R} / P_{e}\left(1-F_{2}\right)$, instead of $\Delta_{R} /\left(1-F_{2}\right)$, since $P_{e}$ varied slightly during measurements at these two energies.] A similar analysis was applied to the false asymmetries $\Delta_{F 1}$ and $\Delta_{F 2}$. If no systematic effects were indicated, as was generally the case, the individual run results for $\Delta_{R} /\left(1-F_{2}\right)$ were combined according to their statistical weights to give an average $A$ [see Eq. (23)] with the uncertainties in $P_{e}, P_{\mathrm{H}}$, and $|\cos \alpha|$ added in quadrature. The average values of $\Delta_{F 1}$ and $\Delta_{F 2}$ were calculated in a similar manner.

\section{B. Review of elastic scattering data}

In this section, covering elastic scattering, and the next, dealing with impact ionization, modifications to the data analysis procedure discussed above are detailed for the few specific instances of nonstatistical behavior of the data. Also discussed are the changes applied to earlier results ${ }^{16-18}$ based upon our present understanding.

Three instances of nonstatistical behavior in the elastic data became evident from consideration of the $\Lambda^{e}(T)$ versus $\Gamma(T)$ plots. In the first instance, corresponding to $4.4 \mathrm{eV}$ (the lowest energy of the ten energies studied in the recent elastic scattering experiment), a linear fit to the data of $\Lambda^{e}(T)$ versus $\Gamma(T)$ resulted in a nonstatistical value of $\chi^{2}$. As a consequence, the measured values of $\Lambda^{e}(T)$ were used to determine $F_{2}^{e}$ for each run, instead of values derived from a linear fit, the normal procedure as described in Sec. IIID. The observed fluctuations in $\Lambda^{e}(T)$ are thought to be due to an increased susceptibility of $\Lambda^{e}(T)$ to small changes in the electron-beam position due to drifts in electronics at the energy of $4.4 \mathrm{eV}$, which was also the lowest energy for which a reasonable signal to background ratio could be maintained. At lower energies, the spatial extent of the electron beam increased due to conservation of phase space, with the result that wallscattered and aperture-scattered events overwhelmed the signal.

The second instance of nonstatistical behavior occurred at an energy of $24.3 \mathrm{eV}$, where the installation of a new $\mathrm{H}$ oven was followed by one run for which $\Lambda^{e}(T)$ deviated $8 \sigma$ from the best-fit line determined by the $\Lambda^{e}(T)$ 's of the remaining runs. This nonstatistical behavior is attributed to oven motion during the first heating. Consequently the elastic and impact ionization data for this one run were discarded. The final instance of nonstatistical behavior occurred for one run at $30.3 \mathrm{eV}$ where an abrupt increase of $\Gamma(T)$ was accompanied by a decrease in $\Lambda^{e}(T)$. This anomalous response was probably caused by a crack that had developed near the orifice of the $\mathbf{H}$ oven. All data acquired following the abrupt change in $\Gamma(T)$ and prior to replacement of the oven were discarded.
The $\chi^{2}$ analysis of the real asymmetry $\Delta_{R} /\left(1-F_{2}^{e}\right)$ for elastic scattering at an energy of $22.2 \mathrm{eV}$ revealed the presence of a possible systematic effect. For one target polarization the calculated reduced $\chi^{2}$ was equal to 2.5 for 10 degrees of freedom (11 runs) and was significantly affected by two runs, one with a $\chi^{2}=7.8$. These two runs were examined with care to determine the possible causes of such large deviations. No instabilities or noise were observed, and the data for both electron polarizations satisfied Poisson statistics, as can be seen in Fig. 19. Faced with what appears to be a statistical quirk, we quote the results of the data at $22.2 \mathrm{eV}$ (as presented in Sec. V A) in three ways-first with no runs discarded, second with one run discarded, and third with two runs discarded. [The value of $A_{90^{\circ}}(1 S \rightarrow 1 S)$ shown in Fig. 22(a) at $22.2 \mathrm{eV}$ is the result obtained with two runs discarded.] The only justification for rejection of data in this case is Chauvenet's criterion, ${ }^{124}$ a prescription for omission of data based on the size of the deviation from the mean.

No systematic effects on the elastic asymmetry resulting from electron polarization reversal were revealed from the $\chi^{2}$ analysis of the false asymmetries $\Delta_{F 1}$ and $\Delta_{F 2}$ for any energy. Both false asymmetries were zero within
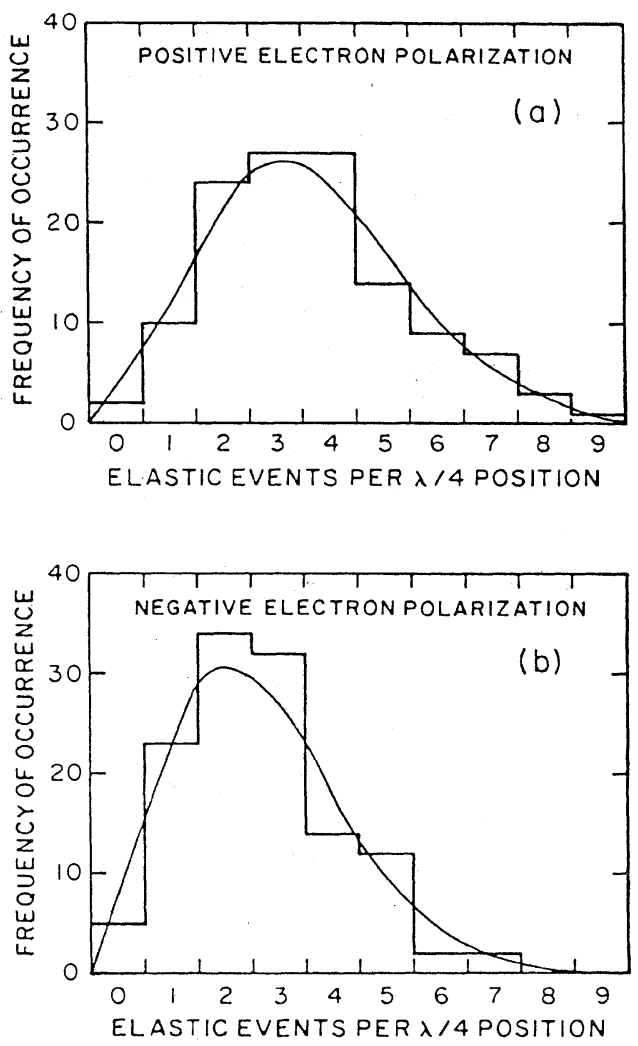

FIG. 19. Frequency of occurrence of $N$ (the number of elastic events) as a function of $N$ recorded for each quarter-waveplate data bin, for incident electron polarization (a) positive and (b) negative. The bar graphs are the data, while the curves are the corresponding Poisson distributions calculated from their mean values. The number of data bins is 124 in each case. The data are taken from the one run for which $\chi^{2}$ for $\Delta_{R} /\left(1-F_{2}^{e}\right)$ is 7.8 . 
statistics for each energy and for each target polarization. Finally, no systematic effects related to hydrogen polarization reversal were found. From a combination of data of all ten energies studied in the recent experiment, the ratio of the asymmetry $\Delta_{R} /\left(1-F_{2}^{e}\right)$ for the "reversed" target polarization direction, denoted REV (hydrogen polarization direction opposite to incident electron momenta), to the asymmetry for the "normal" target polarization direction, denoted NOR (hydrogen polarization direction parallel to incident electron momenta), was determined to be $0.78 \pm 0.17$. The reduced $\chi^{2}$ about unity of this ratio was 1.05 for 10 degrees of freedom, indicating an absence of systematic effects arising from target polarization reversal.

\section{Review of ionization data}

A systematic dependence of the ionization asymmetry on the direction of the magnetic field in the interaction region was observed in the recent work at incident energies of $22.2,27.0$, and $30.3 \mathrm{eV} .{ }^{24}$ The weighted average of the ratio $A_{I}(\mathrm{REV}) / A_{I}(\mathrm{NOR})$ was $1.13 \pm 0.05$ at these three energies; for the remaining three energies at which both NOR and REV data were taken, the average was $1.05 \pm 0.06$. A similar systematic effect was observed in the early work ${ }^{16}$ at the energies 15.0 and $27.0 \mathrm{eV}$ and was attributed at that time to the undetected presence (in the NOR orientation) of a magnetic field component in the interaction region transverse to the electron-beam direction, which reduced the value of $|\cos \alpha|$ in Eq. (23). This explanation was reinforced by the observation that the size of the effect decreased when the longitudinal field in the interaction region was increased from 100 to $200 \mathrm{mG}$. However, since the elastic scattering data, obtained more recently, do not display any such systematic effect, a more likely explanation of the discrepancy in the REV and NOR values for $A_{I}$ lies in an incomplete detector acceptance for ions with large recoil angles. This hypothesis also explains the nonlinearity of $\Lambda^{I}(T)$ as a function of $\Gamma(T)$ (see Sec. IIID) as well as the observation that the ion signal did not scale with the known total ionization cross section in the energy range from 30 to $60 \mathrm{eV}$. Indeed, since the initial momentum and momentum spread of the hot $\mathrm{H}$ beam and the cold $\mathrm{H}_{2}$ beam were approximately equal, the angular distributions of recoil $\mathrm{H}^{+}$ and $\mathrm{H}_{2}{ }^{+}$ions would have been similar if the collision processes of electron impact ionization of $\mathrm{H}$ and $\mathrm{H}_{2}$ are assumed to be roughly similar. Thus it would be expected that $\Lambda^{I}$ and $\bar{\sigma}_{I}(\mathrm{H})$ would both exhibit systematic variations, as was the case. Furthermore, it can be argued that the acceptance would be affected by the position of the beam overlap in the interaction region, which could change upon reversal of the magnetic field, thereby resulting in higher asymmetries for the REV orientation at the energies mentioned above.

In our final analysis, we treated both the NOR and REV data without prejudice since we could not assess a priori whether the higher asymmetries of the REV configuration were due to a more, or less, complete acceptance of the ion detector than for asymmetries recorded in the NOR orientation. As a corollary, we now believe that the treatment of data in the early work, in which all $A_{I}$ (NOR) were corrected upward by $6 \%$, is incorrect. Thus we reanalyzed the early data in accordance with our prescription for the recent data: at those energies where a nonstatistical spread between $A_{I}(\mathrm{NOR})$ and $A_{I}(\mathrm{REV})$ existed, we calculated a weighted average for $A_{I}$ from $A_{I}(\mathrm{NOR})$ and $A_{I}(\mathrm{REV})$. We then increased the uncertainty in $A_{I}$ to include the range of statistical uncertainties in $A_{I}(\mathrm{NOR})$ and $A_{I}(\mathrm{REV})$.

At $30.3 \mathrm{eV}$ of the recent work and $107.0 \mathrm{eV}$ of the early work, there was additional nonstatistical behavior in the real asymmetry for each of the field orientations separately. For these cases the quoted asymmetry is a weighted average of the unweighted average for each orientation, with an uncertainty which includes the range of uncertainty (standard deviation of the mean) for each orientation. In all cases nonstatistical uncertainties were added linearly to the quadrature sum of statistical uncertainties for $P_{e}, P_{\mathrm{H}},\left(1-F_{2}^{I}\right)$, and $|\cos \alpha|$.

In some cases the presence of some uncorrected systematic effects associated with electron polarization reversal or time-dependent experimental parameters are indicated by the nonstatistical reduced $\chi^{2}$ about zero $\left[\chi^{2}(0)\right]$ values for $\Delta_{F 1}$ and $\Delta_{F 2}$. However, the values of $\Delta_{F 1}$ and $\Delta_{F 2}$ themselves are so small that we believe these systematic effects have a negligible influence on the measured values of $A_{I}$.

\section{RESULTS AND DISCUSSION}

In this section we present the results of the measurements of $A_{90^{\circ}}(1 S \rightarrow 1 S)$ and $A_{I}$, and compare them with the various approximation methods discussed in Sec. I B. We also present measurements of the relative spinaveraged elastic differential cross section at $90^{\circ}$, and compare them with the energy dependence found by other researchers. In order to provide a background against which the results can be viewed, we summarize the important experimental operating parameters in Table I. It should be noted that in the early work the electron polarization was generally lower, $P_{e}=0.42-0.65( \pm 6 \%)$ and the electron-beam energy spread larger, $\Delta E$ (FWHM) $=3.0 \mathrm{eV}$.

\section{A. $90^{\circ}$ elastic scattering}

Concurrent with the measurement of the threshold for ionization (see Sec. III C), measurements of the $90^{\circ}$ elastic scattering of unpolarized electrons from atomic hydrogen were obtained for energies from 13.0 to $23.5 \mathrm{eV}$. The procedure for placing these relative spin-averaged elastic data on an absolute differential cross-section scale (involving correction for molecular scattering and normalization to the absolute cross-section value observed by Williams ${ }^{30}$ at $16.5 \mathrm{eV}$ ) is described in detail in Appendix C; with the results of the analysis shown in Fig. 20. The agreement of our results with those of other researchers ${ }^{27,30}$ provides two important tests of the experiment. First, it shows that inelastically scattered electrons were effectively discriminated against by the electron detector filter lens. Second, the results imply that both the electron and hydrogen beams remained stable in position for the duration 


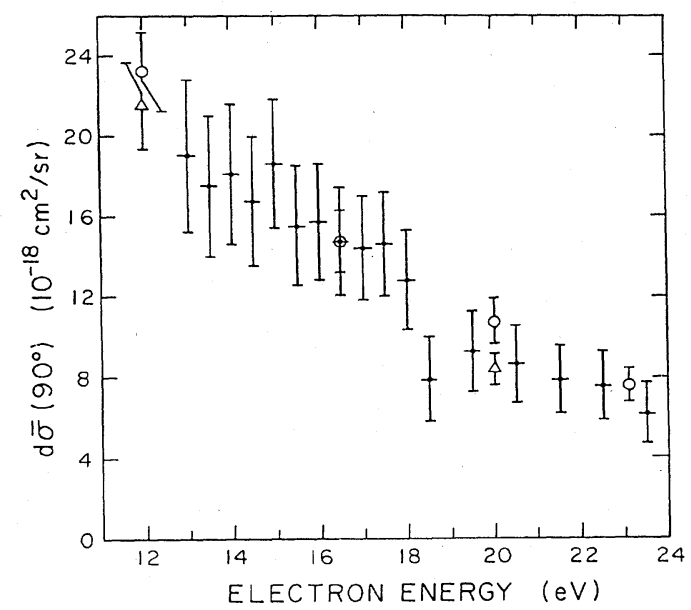

FIG. 20. Measurements of the spin-averaged $90^{\circ}$ elastic differential cross section. The present data (closed circles) have been corrected for molecular scattering and are normalized to the measurement reported by Callaway and Williams (Ref. 30) at $16.5 \mathrm{eV}$. The horizontal bars represent the estimated \pm 0.1 $\mathrm{eV}$ uncertainty in the unpolarized electron beam energy. The typically $\sim 20 \%$ vertical error bars arise from a combination of the $\sim 9 \%$ statistical uncertainty, the $10 \%$ uncertainty in the atomic cross section used for normalization (Ref. 30), and the $25 \%$ uncertainties in the molecular cross sections (Ref. 125). Comparison is made with the measurements of Williams (Ref. 30 , open circles) and of Teubner and co-workers (Ref. 27, triangles).

of these measurements, a condition more stringent than that required for accurate measurements of $A_{90^{\circ}}(1 S \rightarrow 1 S)$.

The measurements of $A_{90^{\circ}}(1 S \rightarrow 1 S)$ reported here were obtained for 12 values of incident electron energy from 4.4 to $30.3 \mathrm{eV}$, including two values, at 8.3 and $10.0 \mathrm{eV}$, from the early measurements. ${ }^{126}$ The number of elastic events recorded at each of the ten energy values of the recent work for each of the two atomic hydrogen polarization directions and for the two pairs of opposite orientations $\left(180^{\circ}\right.$ rotation) of the linear polarizer is shown in bar-graph form in Fig. 21. At each energy, approximately equal numbers of events were obtained for the NOR and REV directions and for each of the two vertical bars, a procedure adopted in order that the asymmetry measurements not be biased with respect to hydrogen polarization direction or linear polarizer orientation.

The results for $A_{90^{\circ}}(1 S \rightarrow 1 S)$ and the elastic scattering false asymmetries $\Delta_{F 1}$ and $\Delta_{F 2}$, determined as discussed in Sec. III A, are presented in Tables II, III, and IV, respectively. The values of $A_{90^{\circ}}(1 S \rightarrow 1 S)$ were determined from the data in accordance with Eqs. (22) and (23) and the analysis given in Secs. IV $A$ and IV $B$. In each case, the uncertainty associated with $A_{90^{\circ}}(1 S \rightarrow 1 S)$ is a one-standard-deviation uncertainty dominated by the statistical uncertainty in $\Delta_{R}$, and includes in quadrature the uncertainties in $P_{e}( \pm 5 \%), P_{\mathrm{H}}( \pm 4 \%),\left(1-F_{2}^{e}\right)( \pm 2 \%)$, and $\cos \alpha( \pm 1.5 \%)$. Results for the respective asymmetries and $\chi^{2}$ analyses are shown for the two hydrogen polarization directions taken together as well as separate-

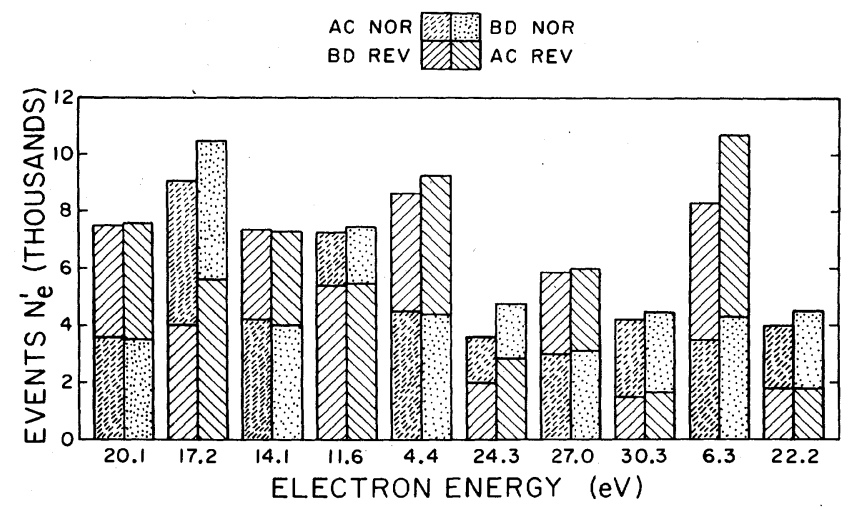

FIG. 21. Number of elastic events $N_{e}^{\prime}$ (background events subtracted) obtained at each energy for relative orientations of the linear polarizer (positions $A, B, C$, and $D$ ) and interaction region magnetic field direction (NOR and REV). The two bars at each energy correspond to the number of events recorded for the two possible relative orientations of the electron and hydrogen polarizations (parallel and antiparallel). The data are presented in chronological order.

$1 y$, as a check for systematic effects. With the exception of the one case at $22.2 \mathrm{eV}$, discussed earlier in Sec. IV B, the present results are statistically distributed. For each of the false asymmetries, seven out of ten of the present values lie within one standard deviation of zero. The larger $\chi^{2}(0)$ for the results at 8.3 and $10.0 \mathrm{eV}$ are seen as evidence for electronic noise which had not been suppressed during these early exploratory runs.

In Fig. 22(a) the measured values of $A_{90^{\circ}}(1 S \rightarrow 1 S)$ are plotted as a function of incident electron energy and compared with the predicted values determined from a number of theoretical approximation methods using published values of singlet and triplet phase shifts or, alternatively, transition amplitudes. The elastic asymmetry was expressed as in Eq. (3), with singlet $|f+g|^{2}$ and triplet $|f-g|^{2}$ cross sections given by

$$
|f \pm g|^{2}=\left|\frac{1}{k} \sum_{l}(2 l+1) T_{l}^{ \pm} P_{l}(\cos \theta)\right|^{2},
$$

where the transition amplitude $T_{l}^{ \pm}$is found from the relation

$$
T_{l}^{ \pm}=\frac{1}{2 i}\left(e^{2 i \delta_{l}^{ \pm}}-1\right)=e^{i \delta_{I}^{ \pm}} \sin \delta_{l}^{ \pm},
$$

$\delta_{l}^{+}\left(\delta_{l}^{-}\right)$being the l-partial-wave singlet (triplet) phase shift. Generally, only the $l=0$ and $l=2$ terms in the sum were included, since for $\theta=90^{\circ}$ only even $l$ contribute and spin-dependent phase shifts and amplitudes have been published only for $l \leq 3$. Even- $l$ spin-independent phase shifts can be calculated for $l \geq 4$ using an expression due to O'Malley et al., ${ }^{127}$ but the effects of higher- $l$ partial waves are small for large-angle scattering, and hence terms involving $l \geq 4$ were neglected.

While most of the predictions for the spin-averaged $90^{\circ}$ elastic cross section $d \bar{\sigma}\left(90^{\circ}\right)$ agree with each other and with the measurements of other researchers, as seen in Fig. 22(b), there is disagreement among the same approximation methods in the predictions of $A_{90^{\circ}}(1 S \rightarrow 1 S)$ in the 
TABLE II. Results of elastic scattering data analysis for real asymmetry $A_{90^{\circ}}(1 S \rightarrow 1 S)$.

\begin{tabular}{|c|c|c|c|c|c|c|}
\hline \multirow{2}{*}{$\begin{array}{c}\text { Energy }^{\mathrm{a}} \\
(\mathrm{eV})\end{array}$} & \multirow{2}{*}{$\begin{array}{l}\text { NOR and } \\
\text { REV }\end{array}$} & \multicolumn{2}{|l|}{$A_{90^{\circ}}(1 S \rightarrow 1 S)$} & \multicolumn{3}{|c|}{$\begin{array}{l}\chi^{2}(\text { av }) / \text { degree of freedom } \\
\text { NOR and }\end{array}$} \\
\hline & & $\mathrm{NOR}^{\mathrm{b}}$ & $\mathrm{REV}^{\mathrm{b}}$ & REV & NOR & REV \\
\hline $4.4(2.5)$ & $+0.003(46)$ & $+0.014(67)$ & $-0.007(64)$ & $\frac{16}{15}$ & $\frac{5}{8}$ & $\frac{11}{6}$ \\
\hline $6.3(2.5)$ & $-0.151(38)$ & $-0.134(64)$ & $-0.158(44)$ & $\frac{17}{17}$ & $\frac{6}{7}$ & $\frac{11}{9}$ \\
\hline $8.3(3.6)^{\mathrm{c}}$ & $-0.192(37)$ & $-0.180(41)$ & $-0.212(51)$ & $\frac{14}{23}$ & $\frac{7}{11}$ & $\frac{6}{11}$ \\
\hline $10.0(5.2)^{\mathrm{c}}$ & $-0.229(64)$ & $-0.186(35)$ & $-0.274(36)$ & $\frac{46}{37}$ & $\frac{6}{7}$ & $\frac{36}{29}$ \\
\hline $11.6(2.7)$ & $-0.281(53)$ & $-0.195(94)$ & $-0.315(58)$ & $\frac{8}{13}$ & $\frac{3}{3}$ & $\frac{3}{9}$ \\
\hline $14.1(2.3)$ & $-0.343(65)$ & $-0.408(76)$ & $-0.227(102)$ & $\frac{13}{13}$ & $\frac{5}{7}$ & $\frac{7}{5}$ \\
\hline $17.2(2.3)$ & $-0.291(56)$ & $-0.177(69)$ & $-0.444(81)$ & $\frac{37}{24}$ & $\frac{15}{11}$ & $\frac{16}{12}$ \\
\hline $20.1(2.7)$ & $-0.197(54)$ & $-0.234(71)$ & $-0.154(76)$ & $\frac{12}{18}$ & $\frac{3}{8}$ & $\frac{9}{9}$ \\
\hline $22.2(2.5)^{\mathrm{d}}$ & $-0.270(54)$ & $-0.260(64)$ & $-0.289(84)$ & $\frac{34}{18}$ & $\frac{25}{10}$ & $\frac{9}{7}$ \\
\hline $22.2(2.5)^{\mathrm{e}}$ & $-0.237(55)$ & $-0.205(66)$ & $-0.289(84)$ & $\frac{26}{17}$ & $\frac{16}{9}$ & $\frac{9}{7}$ \\
\hline $22.2(2.5)^{f}$ & $-0.212(55)$ & $-0.160(69)$ & $-0.289(84)$ & $\frac{21}{16}$ & $\frac{10}{8}$ & $\frac{9}{7}$ \\
\hline $24.3(2.7)$ & $-0.114(66)$ & $-0.186(82)$ & $+0.010(108)$ & $\frac{16}{11}$ & $\frac{5}{4}$ & $\frac{8}{6}$ \\
\hline $27.0(2.7)$ & $-0.200(42)$ & $-0.179(58)$ & $-0.220(56)$ & $\frac{25}{26}$ & $\frac{15}{11}$ & $\frac{10}{14}$ \\
\hline $30.3(2.5)$ & $-0.176(61)$ & $-0.200(77)$ & $-0.139(95)$ & $\frac{14}{16}$ & $\frac{2}{7}$ & $\frac{11}{8}$ \\
\hline All runs ${ }^{f}$ & & & & $\frac{239}{229}$ & $\frac{83}{92}$ & $\frac{138}{125}$ \\
\hline $\begin{array}{l}\text { All runs } \\
\quad(\text { recent work })^{\mathrm{f}}\end{array}$ & & & & $\frac{179}{169}$ & $\frac{70}{74}$ & $\frac{95}{85}$ \\
\hline
\end{tabular}

${ }^{a}$ Electron energies studied in the present work have been corrected upward $0.3 \mathrm{eV}$ from previously published data (Ref. 23), due to a correction in the ionization threshold energy calibration. Electron-beam energy spread (full width at half maximum) in parentheses.

${ }^{b}$ Counting statistics uncertainties only.

${ }^{c}$ Corrected data of Ref. 18.

${ }^{\mathrm{d}}$ No runs discarded. See Sec. IV B.

'One run discarded. See Sec. IV B.

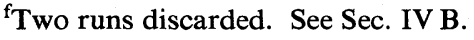

intermediate-energy region. Our results confirm the similar predictions of the variational, ${ }^{46-52}$ close-coupling, ${ }^{71,72}$ pseudostate close-coupling, ${ }^{75}$ and polarized-orbital $^{78}$ methods in the low-energy $(<10 \mathrm{eV})$ region. From 10 to $30 \mathrm{eV}$ the results agree only with the predictions of the $1 S-2 S-2 P$ close-coupling method. ${ }^{72,128}$ However, the agreement is probably fortuitous since this three-state calculation inadequately treats the hydrogen ground-state polarizability, underestimates the spin-averaged cross section at forward angles, and overestimates the widths of resonances.

The algebraic variational pseudostate close-coupling method $^{30,76}$ provides the best agreement between theory and experiment for differential cross sections from 10 to $30 \mathrm{eV}$, but the predictions for $A_{90^{\circ}}(1 S \rightarrow 1 S$ ) (curve $e$ ) do not agree with the experimental results. At large angles $\left(\theta>120^{\circ}\right)$ the algebraic variational method disagrees with cross-section measurements in this energy range: Our experimental results, together with the knowledge that exchange effects are important for large-angle scattering, thus may indicate a problem with the treatment of exchange in this approximation method. As a final observation on the elastic results, we point out that the measurement of $A_{90^{\circ}}(1 S \rightarrow 1 S)$ at $14.1 \mathrm{eV}$ is consistent with pure triplet scattering, reflected by a value of $A_{90^{\circ}}(1 S \rightarrow 1 S)=-\frac{1}{3}$.

\section{B. Impact ionization}

The recent measurements of $A_{I}$ were performed concurrently with the measurements of $A_{90^{\circ}}(1 S \rightarrow 1 S)$ according to the procedure given in Sec. III for seven values of the incident electron energy from 14.1 to $30.3 \mathrm{eV}$. These measurements initially were intended to serve as a consistency check of the experimental method, since several values of $A_{I}$ had been obtained previously in this energy range. However, a comparison between the recent results and the early measurements revealed a small but systematic discrepancy. This discrepancy is now fully accounted for by effects associated with incomplete detector accep- 
TABLE III. Results of elastic scattering data analysis for false asymmetry $\Delta_{F 1}$.

\begin{tabular}{|c|c|c|c|c|c|c|}
\hline \multirow[b]{2}{*}{$\begin{array}{c}\text { Energy }^{\mathrm{a}} \\
(\mathrm{eV})\end{array}$} & \multicolumn{3}{|c|}{$\Delta_{F 1}\left(10^{-3}\right)^{\mathrm{b}}$} & \multicolumn{3}{|c|}{$\chi^{2}(0) /$ degree of freedom ${ }^{c}$} \\
\hline & $\begin{array}{l}\text { NOR and } \\
\text { REV }\end{array}$ & NOR & REV & $\begin{array}{l}\text { NOR and } \\
\text { REV }\end{array}$ & NOR & REV \\
\hline $4.4(2.5)$ & $+1(15)$ & $+18(21)$ & $-15(20)$ & $\frac{10}{16}$ & $\frac{7}{9}$ & $\frac{3}{7}$ \\
\hline $6.3(2.5)$ & $+2(11)$ & $+15(21)$ & $-4(14)$ & $\frac{13}{18}$ & $\frac{4}{8}$ & $\frac{9}{10}$ \\
\hline $8.3(3.6)^{\mathrm{d}}$ & $+1(5)$ & $-4(7)$ & $+6(7)$ & $\frac{12}{24}$ & $\frac{9}{12}$ & $\frac{3}{12}$ \\
\hline $10.0(5.2)^{\mathrm{d}}$ & $0(5)$ & $-7(7)$ & $+7(6)$ & $\frac{70}{38}$ & $\frac{14}{8}$ & $\frac{56}{30}$ \\
\hline $11.6(2.7)$ & $+1(13)$ & $+18(25)$ & $-6(16)$ & $\frac{17}{14}$ & $\frac{1}{4}$ & $\frac{15}{10}$ \\
\hline $14.1(2.3)$ & $+24(16)$ & $+26(20)$ & $+21(26)$ & $\frac{15}{14}$ & $\frac{11}{8}$ & $\frac{4}{6}$ \\
\hline $17.2(2.3)$ & $-8(13)$ & $-20(17)$ & $+10(21)$ & $\frac{26}{25}$ & $\frac{13}{12}$ & $\frac{13}{13}$ \\
\hline $20.1(2.7)$ & $+14(14)$ & $+1(18)$ & $+31(21)$ & $\frac{25}{19}$ & $\frac{11}{9}$ & $\frac{14}{10}$ \\
\hline $22.2(2.5)^{\mathrm{e}}$ & $-6(15)$ & $-7(19)$ & $-4(25)$ & $\frac{14}{19}$ & $\frac{7}{11}$ & $\frac{7}{8}$ \\
\hline $22.2(2.5)^{\mathrm{f}}$ & $-11(16)$ & $-16(20)$ & $-4(25)$ & $\frac{11}{18}$ & $\frac{5}{10}$ & $\frac{7}{8}$ \\
\hline $22.2(2.5)^{\mathrm{g}}$ & $-10(16)$ & $-14(21)$ & $-4(25)$ & $\frac{11}{17}$ & $\frac{4}{9}$ & $\frac{7}{8}$ \\
\hline $24.3(2.7)$ & $-24(18)$ & $-34(25)$ & $-12(27)$ & $\frac{10}{12}$ & $\frac{9}{5}$ & $\frac{2}{7}$ \\
\hline $27.0(2.7)$ & $-26(12)$ & $-30(17)$ & $-23(16)$ & $\frac{27}{27}$ & $\frac{13}{12}$ & $\frac{14}{15}$ \\
\hline $30.3(2.5)$ & $+18(19)$ & $+31(24)$ & $-1(30)$ & $\frac{9}{17}$ & $\frac{5}{8}$ & $\frac{4}{9}$ \\
\hline All runs ${ }^{\mathrm{g}}$ & $0(3)$ & $-4(4)$ & $+3(4)$ & $\frac{245}{241}$ & $\frac{101}{104}$ & $\frac{144}{137}$ \\
\hline $\begin{array}{l}\text { All runs } \\
\quad(\text { recent work })^{\mathrm{g}}\end{array}$ & $-2(4)$ & $-1(6)$ & $-3(6)$ & $\frac{163}{179}$ & $\frac{78}{84}$ & $\frac{85}{95}$ \\
\hline
\end{tabular}

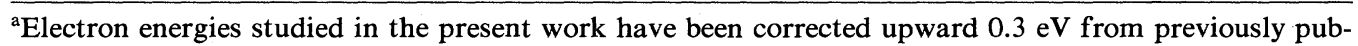
lished data (Ref. 23), due to a correction in the ionization threshold energy calibration. Electron-beam energy spread (full width at half maximum) in parentheses.

${ }^{\mathrm{b}}$ Counting statistics uncertainties only.

${ }^{c} \chi^{2}$ about zero.

${ }^{\mathrm{d} C}$ Corrected data of Ref. 18.

${ }^{\mathrm{e}}$ No runs discarded. See Sec. IV B.

${ }^{f}$ One run discarded. See Sec. IV B.

${ }^{\mathrm{g}}$ Two runs discarded. See Sec. IV B.

TABLE IV. Results of elastic scattering data analysis for false asymmetry $\Delta_{F 2}$.

\begin{tabular}{ccccccc}
\hline $\begin{array}{c}\text { Energy } \\
(\mathrm{eV})\end{array}$ & $\begin{array}{c}\text { NOR and } \\
\text { REV }\end{array}$ & $\begin{array}{c}\Delta_{F 2}\left(10^{-3}\right)^{\mathrm{b}} \\
\text { NOR }\end{array}$ & REV & $\begin{array}{c}\chi^{2}(0) / \text { degree of freedom }^{\mathrm{c}} \\
\text { REV }\end{array}$ & NOR & REV \\
\hline $4.4(2.5)$ & $+11(15)$ & $+17(21)$ & $+6(20)$ & $\frac{16}{16}$ & $\frac{11}{9}$ & $\frac{5}{7}$ \\
$6.3(2.5)$ & $-14(11)$ & $-9(21)$ & $-16(14)$ & $\frac{15}{18}$ & $\frac{7}{8}$ & $\frac{8}{10}$ \\
$8.3(3.6)^{\mathrm{d}}$ & $+10(5)$ & $+17(7)$ & $+4(7)$ & $\frac{37}{24}$ & $\frac{18}{12}$ & $\frac{19}{12}$ \\
$10.0(5.2)^{\mathrm{d}}$ & $+2(5)$ & $+2(7)$ & $+2(6)$ & $\frac{46}{38}$ & $\frac{10}{8}$ & $\frac{36}{30}$ \\
$11.6(2.7)$ & $-2(13)$ & $+31(25)$ & $-14(16)$ & $\frac{14}{14}$ & $\frac{4}{4}$ & $\frac{9}{10}$ \\
$14.1(2.3)$ & $-13(16)$ & $-36(20)$ & $+27(26)$ & $\frac{17}{14}$ & $\frac{10}{8}$ & $\frac{7}{6}$ \\
$17.2(2.3)$ & $+6(13)$ & $+9(17)$ & $+1(21)$ & $\frac{19}{25}$ & $\frac{9}{12}$ & $\frac{10}{13}$ \\
$20.1(2.7)$ & $-28(14)$ & $-33(18)$ & $-22(21)$ & $\frac{15}{19}$ & $\frac{10}{9}$ & $\frac{5}{10}$ \\
$22.2(2.5)^{\mathrm{e}}$ & $+18(15)$ & $+17(19)$ & $+19(25)$ & $\frac{9}{19}$ & $\frac{4}{11}$ & $\frac{4}{8}$ \\
$22.2(2.5)^{\mathrm{f}}$ & $+17(16)$ & $+16(20)$ & $+19(25)$ & $\frac{8}{18}$ & $\frac{4}{10}$ & $\frac{4}{8}$ \\
$22.2(2.5)^{\mathrm{g}}$ & $+15(16)$ & $+13(21)$ & $+19(25)$ & $\frac{8}{17}$ & $\frac{4}{9}$ & $\frac{4}{8}$ \\
$24.3(2.7)$ & $+26(18)$ & $+21(25)$ & $+32(27)$ & $\frac{11}{12}$ & $\frac{2}{5}$ & $\frac{10}{7}$ \\
\hline
\end{tabular}


TABLE IV. (Continued).

\begin{tabular}{lrrrrrr}
\hline \hline $\begin{array}{c}\text { Energy } \\
(\mathrm{eV})\end{array}$ & $\begin{array}{c}\text { NOR and } \\
\text { REV }\end{array}$ & $\begin{array}{c}\Delta_{F 2}\left(10^{-3}\right)^{\mathrm{b}} \\
\text { NOR }\end{array}$ & \multicolumn{5}{c}{ REV } & NOR and & REV & NOR & REV \\
\hline $27.0(2.7)$ & $+7(12)$ & $+9(17)$ & $+6(16)$ & $\frac{23}{27}$ & $\frac{10}{12}$ & $\frac{13}{15}$ \\
$30.3(2.5)$ & $+10(19)$ & $+2(24)$ & $+22(30)$ & $\frac{17}{17}$ & $\frac{3}{8}$ & $\frac{14}{9}$ \\
All runs & $+3(3)$ & $+6(4)$ & $+2(4)$ & $\frac{238}{241}$ & $\frac{98}{104}$ & $\frac{140}{137}$ \\
All runs & $0(4)$ & $0(6)$ & $0(6)$ & $\frac{155}{179}$ & $\frac{70}{84}$ & $\frac{84}{95}$
\end{tabular}

$(\text { recent work })^{\mathrm{g}}$

${ }^{a}$ Electron energies studied in the present work have been corrected upward $0.3 \mathrm{eV}$ from previously published data (Ref. 23), due to a correction in the ionization threshold energy calibration. Electron-beam energy spread (full width at half maximum) in parentheses.

${ }^{b}$ Counting statistics uncertainties only.

${ }^{c} \chi^{2}$ about zero.

${ }^{\mathrm{d}}$ Corrected data of Ref. 18.

eNo runs discarded. See Sec. IV B.

fOne run discarded. See Sec. IV B.

${ }^{\mathrm{g}}$ Two runs discarded. See Sec. IV B.

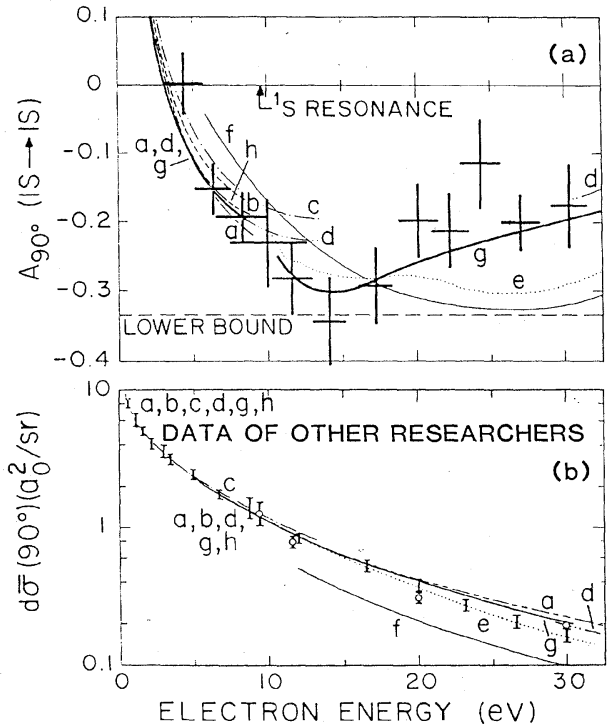

FIG. 22. (a) Measured values of $A_{90^{\circ}}(1 S \rightarrow 1 S)$ as a function of incident energy with representative theoretical predictions. Vertical error bars are one-standard-deviation uncertainties dominated by statistics; horizontal bars indicate the energy spread of the electron beam. Theoretical curves are obtained from information in the following references using the procedures given in the text: $a, 1 S-\overline{2 P}$ pseudostate close coupling (Ref. 77); $b$, variational (Refs. 46, 49, and 50); $c$, polarized orbital (Ref. 78); $d$, variational (Ref. 129); $e$, algebraic variational pseudostate close coupling (Ref 30$) ; f$, exchange-corrected Glauber (Ref. 130); $g$, three-state close coupling (Refs. 71, 72, and 128); $h$, pseudostate close coupling (Ref. 75). (b) Measurements by other researchers of the spin-averaged differential cross section $d \bar{\sigma}\left(90^{\circ}\right)$ compared with the same theoretical results as in (a): solid bars (Refs. 28 and 30); open circles (Ref. 27).

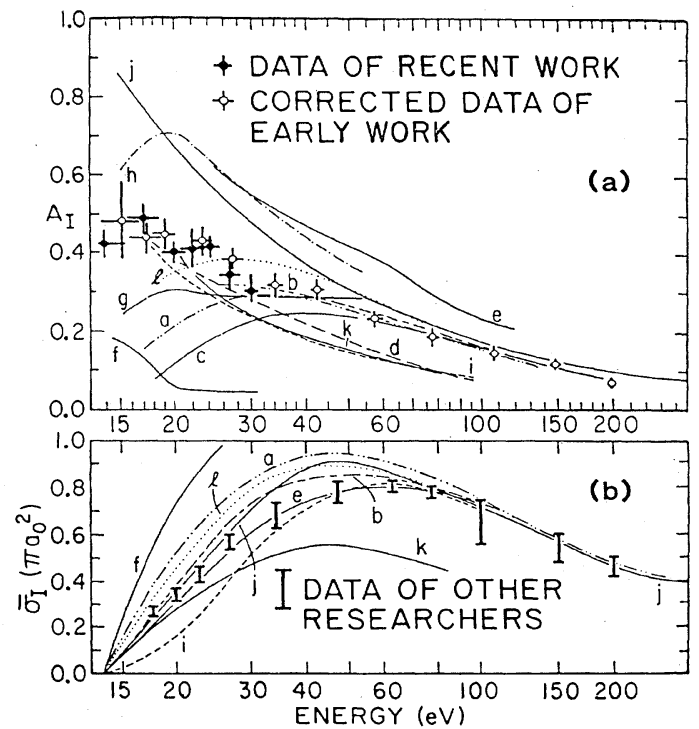

FIG. 23. (a) Measured values of $A_{I}$ as a function of incident electron energy. The recent results are shown as solid circles and the corrected early results (Ref. 16) are shown as open circles. Vertical error bars include statistical and systematic uncertainties. Horizontal bars indicate full width at half maximum energy spread of the electron beam. Theoretical curves are obtained from information in the following references using the procedures given in the text: $a, b, c$, and $d$, Born-exchange (BE) (Refs. 132, 133, 134, and 135, respectively); $e$, BE with maximum interference (Ref. 133); $f$, BE with angle-dependent potential (Ref. 136); $g$ and $h$, spherical average exchange, the latter with maximum interference (Ref. 132); $i$, Glauber exchange (Ref. 135); $j$, modified Born-Oppenheimer (Ref. 137); $k$, pseudostate close coupling (Ref. 138); l, BE (Ref. 139). (b) Experimental values of $\bar{\sigma}_{I}$ obtained by other investigators (Refs. 25 and 106). Vertical bars indicate the spread of the measurements. Theoretical curves are from references given above. 
TABLE V. Results of impact ionization data analysis for real asymmetry $A_{I}$.

\begin{tabular}{|c|c|c|c|c|c|c|}
\hline \multirow[b]{2}{*}{$\begin{array}{l}\text { Energy }^{a} \\
(\mathrm{eV})\end{array}$} & \multirow[b]{2}{*}{$\begin{array}{l}\text { NOR and } \\
\text { REV }\end{array}$} & \multicolumn{2}{|l|}{$A_{I}$} & \multicolumn{3}{|c|}{$\chi^{2}(\mathrm{av}) /$ degree of freedom } \\
\hline & & $\mathrm{NOR}^{\mathrm{b}}$ & $\mathrm{REV}^{\mathrm{b}}$ & $\begin{array}{c}\text { NOR and } \\
\text { REV }\end{array}$ & NOR & REV \\
\hline $14.1(2.3)$ & $0.429\left(\begin{array}{c}+36 \\
-36\end{array}\right)$ & $0.412(34)$ & $0.446(34)$ & $\frac{5}{13}$ & $\frac{4}{7}$ & $\frac{1}{5}$ \\
\hline $15.0(3.2)^{\mathrm{c}}$ & $0.478\left({ }_{-98}^{+101}\right)^{d}$ & $0.420(29)$ & $0.529(27)$ & $\frac{22}{12}$ & $\frac{4}{3}$ & $\frac{11}{8}$ \\
\hline $17.0(3.2)^{\mathrm{c}}$ & $0.434(39)$ & $0.436(18)$ & $0.430(26)$ & $\frac{10}{9}$ & $\frac{6}{3}$ & $\frac{4}{5}$ \\
\hline $17.2(2.3)$ & $0.491\left({ }_{-37}^{+42}\right)$ & $0.494(12)$ & $0.475(28)$ & $\frac{12}{15}$ & $\frac{9}{11}$ & $\frac{2}{3}$ \\
\hline $19.0(3.2)^{\mathrm{c}}$ & $0.435(43)$ & $0.425(20)$ & $0.449(24)$ & $\frac{8}{9}$ & $\frac{5}{4}$ & $\frac{3}{4}$ \\
\hline $20.1(2.7)$ & $0.405(29)$ & $0.405(9)$ & & $\frac{8}{8}$ & $\frac{8}{8}$ & \\
\hline $22.2(2.5)$ & $0.409\left({ }_{-50}^{+56}\right)^{d}$ & $0.381(5)$ & $0.436(5)$ & $\frac{90}{18}$ & $\frac{14}{10}$ & $\frac{5}{7}$ \\
\hline $23.0(3.2)^{\mathrm{c}}$ & $0.428\left({ }_{-42}^{+41}\right)$ & $0.432(21)$ & $0.424(15)$ & $\frac{5}{8}$ & $\frac{2}{3}$ & $\frac{3}{4}$ \\
\hline $24.3(2.7)$ & $0.415\left(\left(_{-42}^{+29}\right)\right.$ & $0.401(12)$ & $0.450(18)$ & $\frac{12}{11}$ & $\frac{1}{4}$ & $\frac{7}{6}$ \\
\hline $27.0(2.7)$ & $0.346\left({ }_{-49}^{+53}\right)^{\mathrm{d}}$ & $0.320(6)$ & $0.369(5)$ & $\frac{73}{25}$ & $\frac{13}{11}$ & $\frac{23}{13}$ \\
\hline $27.0(3.2)^{\mathrm{c}}$ & $0.384\left({ }_{-45}^{+43}\right)^{\mathrm{d}}$ & $0.371(5)$ & $0.397(6)$ & $\frac{76}{62}$ & $\frac{21}{30}$ & $\frac{30}{31}$ \\
\hline $30.3(2.5)$ & $0.302\left({ }_{-30}^{+45}\right)^{\mathrm{d}}$ & $0.293(6)^{\mathrm{e}}$ & $0.324(9)^{\mathrm{e}}$ & $\frac{88}{16}$ & $\frac{20}{7}$ & $\frac{43}{8}$ \\
\hline $34.0(3.2)^{\mathrm{c}}$ & $0.316\left(\left(_{-33}^{+35}\right)\right.$ & $0.332(24)$ & $0.307(18)$ & $\frac{3}{5}$ & $\frac{1}{1}$ & $\frac{2}{3}$ \\
\hline $42.0(3.2)^{\mathrm{c}}$ & $0.310(25)$ & $0.303(8)$ & $0.320(11)$ & $\frac{7}{9}$ & $\frac{4}{4}$ & $\frac{1}{4}$ \\
\hline $57.0(3.2)^{\mathrm{c}}$ & $0.236(21)$ & $0.238(11)$ & $0.233(13)$ & $\frac{9}{8}$ & $\frac{8}{3}$ & $\frac{1}{4}$ \\
\hline $77.0(3.2)^{\mathrm{c}}$ & $0.185\left({ }_{-23}^{+24}\right)$ & $0.185(19)$ & $0.184(31)$ & $\frac{3}{7}$ & $\frac{1}{3}$ & $\frac{2}{3}$ \\
\hline $107.0(3.2)^{\mathrm{c}}$ & $0.143\left({ }_{-17}^{+16}\right)$ & & $0.143(11)^{\mathrm{e}}$ & $\frac{21}{8}$ & & $\frac{21}{8}$ \\
\hline $147.0(3.2)^{\mathrm{c}}$ & $0.118\left({ }_{-16}^{+15}\right)$ & & $0.118(12)$ & $\frac{3}{3}$ & & $\frac{3}{3}$ \\
\hline $197.0(3.2)^{\mathrm{c}}$ & $0.071(15)$ & & $0.071(14)$ & $\frac{1}{3}$ & & $\frac{1}{3}$ \\
\hline $\begin{array}{l}\text { All runs }{ }^{\mathrm{c}} \\
\quad \text { (early work) }\end{array}$ & & & & $\frac{168}{143}$ & $\frac{51}{54}$ & $\frac{82}{80}$ \\
\hline $\begin{array}{l}\text { All runs } \\
\quad(\text { recent work) }\end{array}$ & & & & $\frac{288}{106}$ & $\frac{69}{58}$ & $\frac{80}{42}$ \\
\hline $\begin{array}{l}\text { All runs } \\
\text { (recent work) } \\
\text { excluding } 30.3 \mathrm{eV}\end{array}$ & & & & $\frac{200}{90}$ & $\frac{49}{51}$ & $\frac{37}{34}$ \\
\hline
\end{tabular}

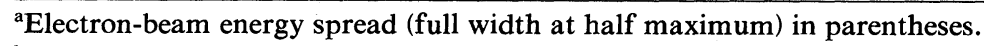

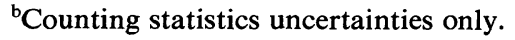

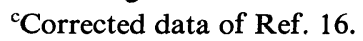

${ }^{\mathrm{d} U n c e r t a i n t y ~ i n c l u d e s ~ s y s t e m a t i c ~ f i e l d ~ e f f e c t s . ~ S e e ~ S e c . ~ I V ~ C . ~}$

${ }^{\mathrm{e} U n w e i g h t e d ~ a v e r a g e ~ w i t h ~ n o n s t a t i s t i c a l ~ u n c e r t a i n t y . ~ S e e . ~ S e c . ~ I V ~ C . ~}$

TABLE VI. Results of impact ionization data analysis for false asymmetry $\Delta_{F 1}$.

\begin{tabular}{ccccccc}
\hline \hline $\begin{array}{c}\text { Energy } \\
(\mathrm{eV})\end{array}$ & $\begin{array}{c}\text { NOR and } \\
\text { REV }\end{array}$ & $\begin{array}{c}\Delta_{F 1}\left(10^{-4}\right)^{\mathrm{b}} \\
\text { NOR }\end{array}$ & REV & $\begin{array}{c}\chi^{2}(0) / \text { degree of freedom }^{\mathrm{c}} \\
\text { NER and }\end{array}$ & NOR & REV \\
\hline $14.1(2.3)$ & $-7(7)$ & $-5(10)$ & $-9(11)$ & $\frac{16}{14}$ & $\frac{12}{8}$ & $\frac{4}{6}$ \\
$15.0(3.2)^{\mathrm{d}}$ & $-117(57)$ & $+31(84)$ & $-245(78)$ & $\frac{15}{13}$ & $\frac{1}{4}$ & $\frac{13}{9}$ \\
$17.0(3.2)^{\mathrm{d}}$ & $+77(42)$ & $+110(50)$ & $-9(80)$ & $\frac{8}{10}$ & $\frac{5}{4}$ & $\frac{2}{6}$ \\
$17.2(2.3)$ & $-7(20)$ & $-14(22)$ & $+21(46)$ & $\frac{8}{16}$ & $\frac{6}{12}$ & $\frac{2}{4}$ \\
$19.0(3.2)^{\mathrm{d}}$ & $+54(36)$ & $+54(47)$ & $+55(57)$ & $\frac{10}{10}$ & $\frac{3}{5}$ & $\frac{8}{5}$ \\
$20.1(2.7)^{\mathrm{e}}$ & $-17(22)$ & $-17(22)$ & & $\frac{5}{9}$ & $\frac{5}{9}$ & \\
$22.2(2.5)$ & $+13(8)$ & $+2(11)$ & $+26(12)$ & $\frac{20}{19}$ & $\frac{13}{11}$ & $\frac{7}{8}$ \\
$23.0(3.2)^{\mathrm{d}}$ & $-69(35)$ & $-53(50)$ & $-84(48)$ & $\frac{16}{9}$ & $\frac{4}{4}$ & $\frac{11}{5}$ \\
$24.3(2.7)$ & $-14(10)$ & $-15(12)$ & $-12(19)$ & $\frac{12}{12}$ & $\frac{4}{5}$ & $\frac{9}{7}$ \\
$27.0(2.7)$ & $+17(12)$ & $+4(18)$ & $+26(15)$ & $\frac{25}{26}$ & $\frac{7}{12}$ & $\frac{18}{14}$ \\
\hline
\end{tabular}


TABLE VI. (Continued).

\begin{tabular}{|c|c|c|c|c|c|c|}
\hline \multirow[b]{2}{*}{$\begin{array}{c}\text { Energy }^{\mathrm{a}} \\
(\mathrm{eV})\end{array}$} & \multicolumn{3}{|c|}{$\Delta_{F 1}\left(10^{-4}\right)^{\mathrm{b}}$} & \multicolumn{3}{|c|}{$\chi^{2}(0) /$ degree of freedom ${ }^{c}$} \\
\hline & $\begin{array}{l}\text { NOR and } \\
\text { REV }\end{array}$ & NOR & REV & $\begin{array}{l}\text { NOR and } \\
\text { REV }\end{array}$ & NOR & REV \\
\hline $27.0(3.2)^{d}$ & $+5(7)$ & $+4(9)$ & $+7(11)$ & $\frac{78}{68}$ & $\frac{49}{36}$ & $\frac{29}{32}$ \\
\hline $30.3(2.5)$ & $+3(8)$ & $+1(11)$ & $+5(12)$ & $\frac{20}{17}$ & $\frac{14}{8}$ & $\frac{7}{9}$ \\
\hline $34.0(3.2)^{\mathrm{d}}$ & $-60(24)$ & $-195(42)$ & $+7(30)$ & $\frac{38}{6}$ & $\frac{34}{2}$ & $\frac{3}{4}$ \\
\hline $42.0(3.2)^{\mathrm{d}}$ & $+3(17)$ & $+22(22)$ & $-29(28)$ & $\frac{8}{10}$ & $\frac{4}{5}$ & $\frac{4}{5}$ \\
\hline $57.0(3.2)^{d}$ & $-6(23)$ & $+18(30)$ & $-43(37)$ & $\frac{26}{9}$ & $\frac{15}{4}$ & $\frac{10}{5}$ \\
\hline $77.0(3.2)^{\mathrm{d}}$ & $+7(47)$ & $+79(56)$ & $-147(82)$ & $\frac{15}{9}$ & $\frac{4}{4}$ & $\frac{10}{5}$ \\
\hline $107.0(3.2)^{\mathrm{d}}$ & $+24(17)$ & $-15(44)$ & $+31(19)$ & $\frac{25}{12}$ & $\frac{8}{5}$ & $\frac{18}{7}$ \\
\hline $147.0(3.2)^{\mathrm{d}}$ & $+13(18)$ & $+25(25)$ & $-2(27)$ & $\frac{11}{8}$ & $\frac{7}{4}$ & $\frac{5}{4}$ \\
\hline $197.0(3.2)^{d}$ & $-26(23)$ & $-5(30)$ & $-55(35)$ & $\frac{12}{8}$ & $\frac{7}{4}$ & $\frac{6}{4}$ \\
\hline $\begin{array}{l}\text { All runs }{ }^{\mathrm{d}} \\
\quad \text { (early work) }\end{array}$ & $+2(5)$ & $+5(7)$ & $-3(8)$ & $\frac{261}{172}$ & $\frac{141}{81}$ & $\frac{119}{91}$ \\
\hline $\begin{array}{l}\text { All runs } \\
\text { (recent work) }\end{array}$ & $0(4)$ & $-4(5)$ & $+8(6)$ & $\frac{107}{113}$ & $\frac{61}{65}$ & $\frac{46}{48}$ \\
\hline
\end{tabular}

${ }^{a}$ Electron-beam energy spread (full width at half maximum) in parentheses.

${ }^{b}$ Counting statistics uncertainties only.

${ }^{c} \chi^{2}$ about zero.

${ }^{\mathrm{d}}$ Corrected data of Ref. 16.

eNormal (NOR) field only.

TABLE VII. Results of impact ionization data analysis for false asymmetry $\Delta_{F 2}$.

\begin{tabular}{|c|c|c|c|c|c|c|}
\hline \multirow[b]{2}{*}{$\begin{array}{c}\text { Energy }^{\mathbf{a}} \\
(\mathrm{eV})\end{array}$} & \multicolumn{3}{|c|}{$\Delta_{F 2}\left(10^{-4}\right)^{\mathrm{b}}$} & \multicolumn{3}{|c|}{$\chi^{2}(0) /$ degree of freedom ${ }^{c}$} \\
\hline & $\begin{array}{c}\text { NOR and } \\
\text { REV }\end{array}$ & NOR & REV & $\begin{array}{c}\text { NOR and } \\
\text { REV }\end{array}$ & NOR & REV \\
\hline $14.1(2.3)$ & $-1(7)$ & $-1(10)$ & $-2(11)$ & $\frac{20}{14}$ & $\frac{10}{8}$ & $\frac{10}{6}$ \\
\hline $15.0(3.2)^{\mathrm{d}}$ & $-16(57)$ & $+75(84)$ & $-94(78)$ & $\frac{9}{13}$ & $\frac{5}{4}$ & $\frac{5}{9}$ \\
\hline $17.0(3.2)^{\mathrm{d}}$ & $-66(42)$ & $-90(50)$ & $-4(80)$ & $\frac{8}{10}$ & $\frac{6}{4}$ & $\frac{2}{6}$ \\
\hline $17.2(2.3)$ & $+16(20)$ & $0(22)$ & $+86(46)$ & $\frac{20}{16}$ & $\frac{10}{12}$ & $\frac{10}{4}$ \\
\hline $19.0(3.2)^{\mathrm{d}}$ & $+98(36)$ & $+88(47)$ & $+113(57)$ & $\frac{18}{10}$ & $\frac{9}{5}$ & $\frac{9}{5}$ \\
\hline $20.1(2.7)^{\mathrm{e}}$ & $+18(22)$ & $+18(22)$ & & $\frac{15}{9}$ & $\frac{15}{9}$ & \\
\hline $22.2(2.5)$ & $+7(8)$ & $+16(11)$ & $0(12)$ & $\frac{21}{19}$ & $\frac{12}{11}$ & $\frac{9}{8}$ \\
\hline $23.0(3.2)^{\mathrm{d}}$ & $-5(35)$ & $-20(50)$ & $+8(48)$ & $\frac{11}{9}$ & $\frac{2}{4}$ & $\frac{8}{5}$ \\
\hline $24.3(2.7)$ & $+2(10)$ & $+3(12)$ & $-1(19)$ & $\frac{9}{12}$ & $\frac{5}{5}$ & $\frac{5}{7}$ \\
\hline $27.0(2.7)$ & $+6(12)$ & $+28(18)$ & $-10(15)$ & $\frac{27}{26}$ & $\frac{8}{12}$ & $\frac{20}{14}$ \\
\hline $27.0(3.2)^{\mathrm{d}}$ & $-10(7)$ & $-16(9)$ & $-3(11)$ & $\frac{79}{68}$ & $\frac{55}{36}$ & $\frac{24}{32}$ \\
\hline $30.3(2.5)$ & $-16(8)$ & $-26(11)$ & $-5(12)$ & $\frac{17}{17}$ & $\frac{10}{8}$ & $\frac{8}{9}$ \\
\hline $34.0(3.2)^{\mathrm{d}}$ & $+32(24)$ & $+110(42)$ & $-7(30)$ & $\frac{9}{6}$ & $\frac{7}{2}$ & $\frac{2}{4}$ \\
\hline $42.0(3.2)^{\mathrm{d}}$ & $+35(17)$ & $+12(22)$ & $+73(28)$ & $\frac{20}{10}$ & $\frac{11}{5}$ & $\frac{10}{5}$ \\
\hline $57.0(3.2)^{\mathrm{d}}$ & $0(23)$ & $-8(30)$ & $+11(37)$ & $\frac{11}{9}$ & $\frac{6}{4}$ & $\frac{6}{5}$ \\
\hline $77.0(3.2)^{\mathrm{d}}$ & $-19(47)$ & $-53(56)$ & $+55(82)$ & $\frac{7}{9}$ & $\frac{4}{4}$ & $\frac{3}{5}$ \\
\hline $107.0(3.2)^{\mathrm{d}}$ & $-6(17)$ & $-30(44)$ & $-2(19)$ & $\frac{6}{12}$ & $\frac{3}{5}$ & $\frac{4}{7}$ \\
\hline
\end{tabular}


TABLE VII. (Continued).

\begin{tabular}{|c|c|c|c|c|c|c|}
\hline \multirow[b]{2}{*}{$\begin{array}{l}\text { Energy }^{a} \\
(e V)\end{array}$} & \multicolumn{3}{|c|}{$\Delta_{F 2}\left(10^{-4}\right)^{\mathrm{b}}$} & \multicolumn{3}{|c|}{$\chi^{2}(0) /$ degree of freedom ${ }^{c}$} \\
\hline & $\begin{array}{c}\text { NOR and } \\
\text { REV }\end{array}$ & NOR & REV & $\begin{array}{c}\text { NOR and } \\
\text { REV }\end{array}$ & NOR & REV \\
\hline $147.0(3.2)^{\mathrm{d}}$ & $+20(18)$ & $+40(25)$ & $-3(27)$ & $\frac{7}{8}$ & $\frac{6}{4}$ & $\frac{1}{4}$ \\
\hline $197.0(3.2)^{\mathrm{d}}$ & $+7(23)$ & $+15(30)$ & $-3(35)$ & $\frac{3}{8}$ & $\frac{2}{4}$ & $\frac{1}{4}$ \\
\hline $\begin{array}{l}\text { All runs }{ }^{\mathrm{d}} \\
\quad \text { (early work) }\end{array}$ & $+1(5)$ & $-3(7)$ & $+5(8)$ & $\frac{189}{172}$ & $\frac{116}{81}$ & $\frac{75}{91}$ \\
\hline $\begin{array}{l}\text { All runs } \\
\text { (recent work) }\end{array}$ & $0(4)$ & $+1(5)$ & $-2(6)$ & $\frac{130}{113}$ & $\frac{69}{65}$ & $\frac{60}{48}$ \\
\hline
\end{tabular}

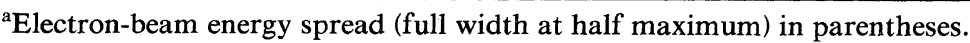

${ }^{\mathrm{b}}$ Counting statistics uncertainties only.

${ }^{c} \chi^{2}$ about zero.

${ }^{\mathrm{d}}$ Corrected data of Ref. 16.

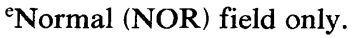

tance, beam-related background signals, and a presence in the Mott measurements of an elastic peak due to Formvar scattering and a polarization dependence in the inelastic background, all of which had not been considered properly in the early work.

The results for $A_{I}$ and the ionization false asymmetries $\Delta_{F 1}$ and $\Delta_{F 2}$ for both the recent data and the corrected earlier data are listed, respectively, in Tables V, VI, and VII. Included in the tables are the results for the two hydrogen polarizations taken together as well as separately, with a $\chi^{2}$ analysis of the averages. The values of $A_{I}$ were determined from the ionization data in accordance with Eqs. (22) and (23) and the analysis discussed in Secs. IV A and IV C. The uncertainty associated with $A_{I}$ ("Both fields" in Table V) includes in quadrature the uncertainties in $\Delta_{R},\left(1-F_{2}^{I}\right), P_{e}( \pm 5 \%), P_{\mathrm{H}}( \pm 4 \%)$, and $\cos \alpha$ $( \pm 1.5 \%)$, and includes linearly those nonstatistical uncertainties described in Sec. IV C. The early results have been revised to include the effects of beam-related background signal (see Sec. III D), incomplete ion acceptance (see Sec. IV C), and the corrected value of electron polarization. ${ }^{119}$

At the energies where the data exhibited nonstatistical behavior, as evidenced by large $\chi^{2}$ (av) in Table V, the uncertainty has been increased according to the analysis procedure given in Sec. IV C. The values of the false asymmetries given in Table VI and VII are very small and generally consistent with zero. As was stated earlier, the influence on $A_{I}$ of possible systematic effects associated with a time-dependent parameter, as indicated in some cases by the $\chi^{2}$ analysis of $\Delta_{F 1}$ and $\Delta_{F 2}$, is considered to be so small that it has been neglected.

The results for $A_{I}$ as a function of energy are shown graphically in Fig. 23(a) along with a number of predictions based on different approximations. The predicted dependence of $A_{I}$ given by curves $j$ and $k$ was calculated from published values of the total singlet and triplet ionization cross sections using Eq. (10). All other theoretical curves were obtained from published values of $\bar{\sigma}_{I}$ [Eq. (13)], the spin-averaged total ionization cross section with exchange included, and $\sigma_{I}^{*}$, the total ionization cross sec- tion with exchange neglected, by combining Eqs. (11) and (14) to give

$$
A_{I}=\frac{\left(\sigma_{I}^{*}-\bar{\sigma}_{I}\right)}{\bar{\sigma}_{I}} .
$$

In Fig. 23(b) the $\bar{\sigma}_{I}$ measurements of other researchers are compared with the same theoretical predictions. Several conclusions can be drawn from the figure. First, while most of the calculations agree at least in shape with the observed energy dependence of $\bar{\sigma}_{I}$, the calculations which agree with $A_{I}$ above about twice the ionization energy disagree below that energy, and those which agree at low energy with our results disagree at higher energies. Second, the recent measurements of $A_{I}$ agree with the corrected results of the early measurements. Third, $A_{I}$ is roughly energy independent within the first few eV above threshold, a conclusion reinforced by similar results reported by other groups in impact ionization of potassium, ${ }^{19}$ lithium, ${ }^{21,131}$ and sodium. ${ }^{19,22,131}$ The results for $A_{I}$ of hydrogen reported here, together with the data of the alkali metals obtained with improved energy resolution, support the conclusions of Greene and $\mathrm{Rau}^{104}$ that $A_{I}$ need not be unity at threshold.

\section{Future work}

The application of new polarized-electron-beam and hydrogen-beam technologies will soon mark the maturation of experiments in elastic, inelastic, and ionization processes which can be easily studied using experimental methods similar to the present method. ${ }^{140}$ Indeed, the discrepancies between theoretical predictions and the results reported here suggest several areas of research in polarized electron-hydrogen collisions which might be pursued in the future. Measurements of $A_{I}$ in the thresholdenergy region with a polarized-electron-beam energy resolution of $\leqslant 30 \mathrm{meV}$, for example, are needed to provide information about the dynamics of two-electron escape and to test further the different threshold law predictions. In the case of elastic scattering, the predictions in the 
theoretically difficult intermediate-energy region can be tested more thoroughly by measurements of $A(1 S \rightarrow 1 S)$ at additional scattering angles and with higher statistical accuracy. In addition, the ${ }^{1} S$ and ${ }^{3} P$ resonances below the $n=2$ threshold should produce structure in the energy dependence of $A(1 S \rightarrow 1 S)$ in the region between 9 and 10 $\mathrm{eV}$, if the energy resolution is of the order of $30 \mathrm{meV}$. Such measurements in the resonance region will furnish an additional experimental test of the predictions of the close-coupling and projection-operator methods used to calculate the positions and widths of the resonances. For both elastic and ionization processes, it is also of interest to determine the energy above which exchange processes become negligible ( $\boldsymbol{A}=0$ ).

A complete account of the scattering of electrons from hydrogen atoms requires the determination of the singlet and triplet elements of the full $T$ matrix, including all excited states. With the use of new polarized beam technologies, the measurement of the $1 S \rightarrow 2 S$ and $1 S \rightarrow 2 P$ matrix elements in particular appear within reach. Measurement of $A(1 S \rightarrow 2 P)$ can be accomplished by the detection of prompt Lyman- $\alpha$ photons emitted from the interaction region. The measurement of $A(1 S \rightarrow 2 S)$ is more complicated, but might be accomplished through the application of a modulated $\mathrm{rf}$ field in the interaction region and the observation of Lyman- $\alpha$ photons. Such an approach would determine separately $A(1 S \rightarrow 2 S)$ and $A(1 S \rightarrow 2 P)$, although the accuracy of these measurements would be degraded somewhat by cascade effects from higher-lying states.

It should be noted that the measurement of $A(1 S \rightarrow 2 S)$ would complete the experimental determination of the corresponding direct and exchange amplitudes (to within the sign of the relative phase angle), since total spinaveraged and exchange cross sections were measured previously. ${ }^{26}$ In the case of $A(1 S \rightarrow 2 P)$ the theoretical description contains four amplitudes, $f_{0}$ and $g_{0}$, the direct and exchange amplitudes for excitation of the $m_{l}=0$ magnetic sublevel, and $f_{1}$ and $g_{1}$, the direct and exchange amplitudes for excitation of the $m_{l}= \pm 1$ sublevels. A complete study of $2 P$ excitation thus involves the determination of seven quantities-four magnitudes and three relative phases. The total $1 S-2 P$ excitation cross-section asymmetry is given by

$$
A(1 S \rightarrow 2 P)=\frac{\int d \Omega\left[\operatorname{Re}\left(f_{0}^{*} g_{0}\right)+2 \operatorname{Re}\left(f_{1}^{*} g_{1}\right)\right]}{\bar{\sigma}(1 S \rightarrow 2 P)},
$$

where the numerator is integrated over all angles of the scattered electron and $\bar{\sigma}(1 S \rightarrow 2 P)$ is the total spinaveraged cross section. As can be seen, a complete separation of the amplitudes is not achieved. However, if electron-photon coincidence techniques ${ }^{141-143}$ are combined with polarized incident beams, for example, the excitation to different $m_{l}$ sublevels can be separated and the quantities $\operatorname{Re}\left(f_{0}^{*} g_{0}\right) /\left(d \bar{\sigma}_{0} / d \Omega\right)$ and $\operatorname{Re}\left(f_{1}^{*} g_{1}\right) /\left(d \bar{\sigma}_{1} / d \Omega\right)$ can be determined, where $d \bar{\sigma}_{0} / d \Omega$ and $d \bar{\sigma}_{1} / d \Omega$ are the respective spin-averaged differential cross sections for excitations of the $m_{l}=0$ and $m_{l}= \pm 1$ sublevels. Moreover, it should be noted that electron-photon coincidence measurements are not affected by cascade processes, since the detection of electrons which have lost $10.2 \mathrm{eV}$ energy is done in coincidence with Lyman- $\alpha$ photons.

The viability of these proposed experiments relies on the development of sources of polarized electrons and polarized hydrogen atoms more intense than those used in the present experiment. The electron source must have far better energy resolution while retaining the feature of optical polarization reversal. Within the last eight years such an electron source has been developed. ${ }^{144,145}$ The GaAs source, as it is known, is already being used in a number of laboratories for atomic collision research. The source produces longitudinally polarized electrons $\left(P_{e} \sim 0.4\right)$ in photoemission from single-crystal GaAs by circularly polarized light, with much higher intensities (typically several $\mu A$ ) and higher intrinsic energy resolution (30-130 meV) than the Fano source, yet with the optical reversibility of $P_{e}$ found to be so important in the measurements reported here. Atomic hydrogen sources based on the dissociation of $\mathrm{H}_{2}$ in a radio-frequency discharge have also reached an advanced stage of development. These sources provide highly directional beams with high dissociation fractions $(>0.9)$, high densities $\left(10^{12}\right.$ atoms $/ \mathrm{cm}^{3}$ at $1 \mathrm{~cm}$ distance from source), and excellent beam stability for extended periods of time. ${ }^{146}$

The technology of electron polarimetry, which has progressed substantially during the last ten years, will also broaden the scope of future measurements. The use of a compact electron polarimeter would permit the separate determination of direct and exchange amplitudes by measuring the change in polarization produced in polarized electron-polarized hydrogen scattering. Furthermore, by studying the spin dependence of the superelastic scattering of electrons from metastable hydrogen (the time-reversed process to $1 S \rightarrow 2 S$ excitation), information about the $2 S$ excitation amplitudes could be obtained without the effects of cascade. In addition to these various experiments with hydrogen, analogous measurements could be performed with the alkali-metal atoms, and perhaps someday with other polarizable species.

\section{ACKNOWLEDGMENTS}

We gratefully acknowledge the contributions of Professor G. Baum, Dr. J. S. Ladish, Dr. C. W. Tu, and Mr. F. C. Tang, and the technical assistance of $\mathbf{J}$. Brosious, $\mathbf{R}$. Broughton, D. Constantino, A. Disco, L. Trudell, and the Yale University Gibbs Laboratory instrumentation shop staff. This research was supported in part by the National Science Foundation under Grant No. PHY-81-05035 at Yale University, by the City University of New York under Grant No. PSC-CUNY-RF-13414, and by the Deutsche Forschungsgemeinschaft. One of us (M.S.L.) acknowledges support by the Alfred P. Sloan Foundation.

\section{APPENDIX A: DERIVATION OF RELATIONSHIP BETWEEN $\Lambda(T)$ AND $\Gamma(T)$}

The linear relationship between $\Lambda(T)$ and $\Gamma(T)$ can be derived as follows. Let $\rho_{1}^{\prime}$ and $\rho_{2}^{\prime}$ be the atomic and molecular densities, respectively, of the beam in the QMA ionizer region, and let $\rho_{1}$ and $\rho_{2}$ be the respective densities of the beam constituents in the interaction region. Fur- 
ther, let $\alpha_{1}$ and $\alpha_{2}$ be the detection probabilities for $\mathrm{H}$ and $\mathrm{H}_{2}$ by the QMA and let $\alpha_{12}$ represent the probability of dissociative ionization of $\mathrm{H}_{2}$ by the QMA ionizer and subsequent detection of $\mathrm{H}^{+}$. Finally, let $\beta_{1}$ and $\beta_{2}$ represent the probabilities of detecting scattering events arising from $\mathrm{H}$ and $\mathrm{H}_{2}$, respectively. Then, $Q_{1}(T)$ and $Q_{2}(T)$, the QMA atomic and molecular signals, respectively, can be written as

$$
Q_{1}(T)=\alpha_{1} \rho_{1}^{\prime}(T)+\alpha_{12} \rho_{2}^{\prime}(T)
$$

and

$$
Q_{2}(T)=\alpha_{2} \rho_{2}^{\prime}(T),
$$

and Eq. (28) takes the form

$$
N^{\prime}(T)=\beta_{1} \rho_{1}(T)+\beta_{2} \rho_{2}(T) .
$$

It then follows from Eqs. (30), (A2), and (A3) that

$$
\Lambda(T)=\frac{\beta_{1}}{\alpha_{2}} \frac{\rho_{1}(T)}{\rho_{2}^{\prime}(T)}+\frac{\beta_{2}}{\alpha_{2}} \frac{\rho_{2}(T)}{\rho_{2}^{\prime}(T)}
$$

and from Eqs. (33), (A1), and (A2) that

$$
\Gamma(T)=\frac{\alpha_{1}}{\alpha_{2}} \frac{\rho_{1}^{\prime}(T)}{\rho_{2}^{\prime}(T)}+\frac{\alpha_{12}}{\alpha_{2}} .
$$

Since $\rho_{2}^{\prime}(T)$ is related to $\rho_{2}(T)$ by a geometrical factor which is independent of $T$, the last term in Eq. (A4) can be written as a constant and is in fact equal to $\Lambda\left(T_{c}\right)$. If Eq. (A5) is then solved for $\rho_{2}^{\prime}(T)$ and the result substituted in Eq. (A4), $\Lambda(T)$ can be expressed as

$\Lambda(T)=\frac{\beta_{1}}{\alpha_{1}} \frac{\rho_{1}(T)}{\rho_{1}^{\prime}(T)} \Gamma(T)-\frac{\alpha_{12} \beta_{1}}{\alpha_{1} \alpha_{2}} \frac{\rho_{1}(T)}{\rho_{1}^{\prime}(T)}+\Lambda\left(T_{c}\right)$.

Now, $\rho_{1}^{\prime}(T)$ and $\rho_{1}(T)$ depend on $T$ not only through the relationship of the dissociation fraction to $T$ but also through the velocity-dependent focusing of the hexapole. However, the ratio $\rho_{1}(T) / \rho_{1}^{\prime}(T)$ is independent of the dissociation fraction. Because of the distances involved (30 $\mathrm{cm}$ from hexapole exit to interaction region and $90 \mathrm{~cm}$ from hexapole exit to QMA ionizer region), the ratio $\rho_{1}(T) / \rho_{1}^{\prime}(T)$ can also be assumed to be independent of the velocity of the hydrogen beam as a first approximation. Equation (A6) can then be expressed in the desired form:

$$
\Lambda(T)=a_{1} \Gamma(T)+a_{0},
$$

where $a_{0}$ and $a_{1}$ are constants.

\section{APPENDIX B: INFLUENCE OF BEAM-RELATED BACKGROUND ASYMMETRIES ON THE ANALYSIS OF $A_{I}$ DATA}

By extending the development presented in Secs. III A and IIID, the effect of the asymmetry in the ionization background on the determination of $A_{I}$ can be quantified. Let $N=S+B_{g}$ and $B=b+B_{g}$ be the respective events recorded during the $\mathrm{ON}$ and OFF gates, where $S$ and $b$ are the hydrogen-beam-related events occurring during the $\mathrm{ON}$ and OFF gates, respectively, and $B_{g}$ represents events which are not beam related. (The OFF signals have been doubled, as in Sec. III A.) Then $N^{\prime}$ is given by

$$
N^{\prime}=N-B=S-b,
$$

showing that the true signal $S$ is larger than the difference $N^{\prime}$ in the observed ON and OFF events by an amount $b$. The original expression for $\Delta_{R}$, Eq. (22), becomes

$\Delta_{R}= \pm\left[S_{02}-S_{13}-\left(b_{02}-b_{13}\right)\right] /\left[S_{02}+S_{13}-\left(b_{02}+b_{13}\right)\right]$.

Since it is assumed that the beam-related asymmetries $\left(S_{02}-S_{13}\right) /\left(S_{02}+S_{13}\right)$ and $\left(b_{02}-b_{13}\right) /\left(b_{02}+b_{13}\right)$ are equal, Eq. (B2) becomes

$$
\Delta_{R}= \pm\left(S_{02}-S_{13}\right) /\left(S_{02}+S_{13}\right) \text {, }
$$

which is the correct expression for $\Delta_{R}$, thus demonstrating that $b$ has no effect on $\Delta_{R}$. However, the original expression for $F_{2}(T)$, given in Eq. (32), becomes

$F_{2}(T)=\frac{\Lambda\left(T_{c}\right)}{\Lambda(T)}=\frac{S\left(T_{c}\right)-b\left(T_{c}\right)}{Q_{2}\left(T_{c}\right)} \frac{Q_{2}(T)}{S(T)-b(T)}$,

which reduces to the correct expression,

$$
F_{2}(T) \equiv \frac{S\left(T_{c}\right)}{Q_{2}\left(T_{c}\right)} \frac{Q_{2}(T)}{S(T)},
$$

only if either

$$
b\left(T_{c}\right)=b(T)=0
$$

or

$$
b\left(T_{c}\right) / b(T)=S\left(T_{c}\right) / S(T) .
$$

Since $b$ depends on the velocity and velocity spread of the beam and the timing constraints (the velocity and velocity spread of a $\mathrm{H}$ beam at $T=2800 \mathrm{~K}$ are twice that of $\mathbf{H}_{2}$ beam at $T_{c}=1400 \mathrm{~K}$ ), while $S$ is not affected by the timing constraints, Eq. (B7) cannot be said to be strictly true but may still be approximately correct. For this reason, and in view of the fact that the correct expression for $F_{2}^{I}$ [Eq. (B5)] cannot be evaluated $\left[S\left(T_{c}\right)\right.$ and $b\left(T_{c}\right)$ are not uniquely determined by the data], $F_{2}^{I}$ was obtained for each run using the original expression [Eqs. (32) or (B4)]. Then at each energy the uncertainty associated with $F_{2}^{I}$ was increased to include two extreme values determined from the correct expression [Eq. (B5), rewritten here using the identity $\left.S=N^{\prime}+b\right]$,

$$
F_{2}(T)=\frac{N^{\prime}\left(T_{c}\right)+b\left(T_{c}\right)}{Q_{2}\left(T_{c}\right)} \frac{Q_{2}(T)}{N^{\prime}(T)+b(T)},
$$

for the two possible extreme values of $b\left(T_{c}\right)$,

$$
b\left(T_{c}\right)=0
$$

or

$$
b\left(T_{c}\right)=B\left(T_{c}\right) b(T) / B(T) .
$$

The ratio $b\left(T_{c}\right) / B\left(T_{c}\right)$ is assumed to be less than or equal to $b(T) / B(T)$, since the timing gates were originally set using a cold $\mathrm{H}_{2}$ beam. The net effect of the background asymmetry $[b(T) \neq 0]$ was an increase in the $F_{2}^{I}$ uncertainties, resulting in an $\sim 5 \%$ increase in the $A_{I}$ uncertainties. 


\section{APPENDIX C: ANALYSIS AND NORMALIZATION OF ELASTIC CROSS-SECTION MEASUREMENTS}

As reported in Sec. V A, measurements of $90^{\circ}$ elastic scattering of unpolarized electrons from atomic hydrogen were recorded for incident energies from 13.0 and $23.5 \mathrm{eV}$. If the assumptions are made that the electron-beam geometry and detector efficiency did not vary appreciably over the above energy range, then these data provide results for the relative spin-averaged elastic differential cross section. Through the following analysis, these cross section results can be placed on an absolute scale.

The presence of both atomic and molecular species in the hydrogen beam dictates that the number of elastic events $N^{\prime}(E)$ as a function of incident electron energy $E$ be represented by

$$
N^{\prime}(E)=\gamma\left[\alpha \rho_{1} d \bar{\sigma}_{1}(E)+\beta \rho_{2} d \sigma_{2}(E)\right],
$$

where $d \bar{\sigma}_{1}(E)$ and $d \sigma_{2}(E)$ are the respective $90^{\circ}$ elastic differential cross sections for atomic and molecular targets, $\rho_{1}$ and $\rho_{2}$ are the respective atomic and molecular densities in the interaction volume, $\alpha$ and $\beta$ are timedependent factors that are defined below, and $\gamma$ is a constant containing all other common factors. The atomic and molecular densities were determined by measuring the fraction $F_{2}$ of events due to $\mathrm{H}_{2}$ (Sec. III D) at an energy of $16.5 \mathrm{eV}$. Since $F_{2}$ is given by

$$
F_{2}=\frac{\rho_{2} d \sigma_{2}(16.5 \mathrm{eV})}{\rho_{1} d \bar{\sigma}_{1}(16.5 \mathrm{eV})+\rho_{2} d \sigma_{2}(16.5 \mathrm{eV})},
$$

Eqs. (C1) and (C2) determine $\rho_{1}$ and $\rho_{2}$ (to within the common factor $\gamma)$ provided $d \bar{\sigma}_{1}(16.5 \mathrm{eV})$ and $d \sigma_{2}(16.5$ $\mathrm{eV})$ are known.

During these unpolarized electron measurements, which spanned approximately one hour, the QMA atomic and molecular signals were observed to decrease with time. Since changes in the QMA signals were assumed to reflect corresponding changes in beam densities in the interactions region, the factors $\alpha$ and $\beta$ were taken to be directly proportional to the QMA $\mathbf{H}$ and $\mathbf{H}_{2}$ signals, respectively, and were set equal to unity at $16.5 \mathrm{eV}$.

The expressions for $\rho_{1}$ and $\rho_{2}$, derived from (C1) and (C2), are

$$
\rho_{1}=\frac{N^{\prime}(16.5 \mathrm{eV})\left(1-F_{2}\right)}{\gamma d \bar{\sigma}_{1}(16.5 \mathrm{eV})}
$$

and

$$
\rho_{2}=\frac{N^{\prime}(16.5 \mathrm{eV}) F_{2}}{\gamma d \sigma_{2}(16.5 \mathrm{eV})} .
$$

Substitution of (C3) and (C4) into (C1) then gives $d \bar{\sigma}_{1}(E)$ at all other energies:

$$
\begin{aligned}
d \bar{\sigma}_{1}(E)= & \frac{d \bar{\sigma}_{1}(16.5 \mathrm{eV})}{\alpha\left(1-F_{2}\right)} \\
& \times\left(\frac{N^{\prime}(E)}{N^{\prime}(16.5 \mathrm{eV})}-\beta F_{2} \frac{d \sigma_{2}(E)}{d \sigma_{2}(16.5 \mathrm{eV})}\right) .
\end{aligned}
$$

Absolute values of $d \bar{\sigma}_{1}$ can be obtained from Eq. (C5) with the use of absolute values of $d \bar{\sigma}_{1}(16.5 \mathrm{eV})$ and $d \sigma_{2}(E)$ from other researchers. For $d \bar{\sigma}_{1}(16.5 \mathrm{eV})$ the value measured by Williams, ${ }^{28,30} d \bar{\sigma}_{1}(16.5 \mathrm{eV})$ $=0.147(15) \times 10^{-16} \mathrm{~cm}^{2} / \mathrm{sr}$, was used in these calculations.

Absolute measurements of electron-molecular hydrogen elastic differential cross sections $d \sigma_{2}(E)$ are less well known. In addition, in the present experiment electrons which lost up to $5 \mathrm{eV}$ energy were detected. This threshold was sufficient to reject electrons inelastically scattered from atomic hydrogen (inelastic energy loss $\geq 10.2 \mathrm{eV}$ ). From molecular hydrogen, however, both rotational excitation (energy loss $\sim 0.08 \mathrm{eV}$ for $\Delta j=+2$ ) and vibrational excitation (energy loss $\sim 0.52 \mathrm{eV}$ for $\Delta v=+1$ ) could be included in the signal. Differential cross sections for rotational, vibrational, and rotational-vibrational excitation of room-temperature molecular hydrogen by electron impact have been measured by several groups. ${ }^{147}$ Linder and Schmidt ${ }^{148}$ found that, at $90^{\circ}$ and $E=10.8 \mathrm{eV}$,

$$
\begin{aligned}
d \sigma_{2}(v=0 \rightarrow 1, j=1 \rightarrow 3) & \simeq d \sigma_{2}(v=0 \rightarrow 1, \Delta j=0) \\
& \leq 0.01 d \sigma_{2}(\text { elastic }) .
\end{aligned}
$$

At $80^{\circ}$ over the energy range from 10 to $20 \mathrm{eV}$, Trajmar et al. ${ }^{149}$ reported that

$$
d \sigma_{2}(v=0 \rightarrow 2) \leq 0.001 d \sigma_{2}(\text { elastic })
$$

and

$$
d \sigma_{2}(v=0 \rightarrow 1) \leq 0.01 d \sigma_{2}(\text { elastic }) .
$$

For our measurements vibrational excitation was therefore neglected. Linder and Schmidt also measured rotational excitation at $90^{\circ}$ and $10.0 \mathrm{eV}$ and determined that

$$
d \sigma_{2}(\Delta v=0, j=1 \rightarrow 3) \simeq 0.15 d \sigma_{2} \text { (elastic) . }
$$

Srivastava et al. ${ }^{150}$ found that the $j=1 \rightarrow 3$ transition was the strongest rotational transition and that at $90^{\circ}$,

$0.15 \leq d \sigma_{2}(\Delta v=0, j=1 \rightarrow 3) / d \sigma_{2}($ elastic $) \leq 0.45$,

as the incident energy varied from 10 to $40 \mathrm{eV}$. Other rotational excitation transitions were too weak for reliable quantitative measurement. Of course, the initial statistical population of the rotational levels of $\mathbf{H}_{2}$ was different for the room-temperature $\mathbf{H}_{2}$ beams of other researchers and for our beam with temperature $T=2800 \mathrm{~K}$. However, no measurements of rotational excitation other than the $j=1 \rightarrow 3$ transition exist in the literature. Consequently, the available room-temperature $\mathbf{H}_{2}$ differential cross-section data were used.

It should be noted, however, that only two groups have measured $e^{-}-\mathrm{H}_{2}$ differential cross sections in the 10-30eV range needed for our analysis. Shyn and Sharp, ${ }^{117}$ who claim that the inelastic $j=1 \rightarrow 3$ rotational excitation was resolved in their experiment, quote an uncertainty in their results of $\pm 13 \%$. The data of Srivastava et al., ${ }^{116}$ renormalized to the more accurate $\mathrm{He}$ elastic cross sections of Register et al., ${ }^{151}$ have a quoted uncertainty of $\pm 15 \% .{ }^{147}$ Their cross sections include the $j=1 \rightarrow 3$ rotational excita- 
tion. More recently Trajmar $^{125}$ has reported that the data of Srivastava et al. ${ }^{116}$ are believed to be accurate only to $\pm 25 \%$ at larger angles. Therefore, interpolated values of $d \sigma_{2}(E)$ were determined from both sets of published data and were used in Eq. (C5) to arrive at the results given in
Sec. V A for $d \bar{\sigma}_{1}(E)$. The typically $\sim 20 \%$ uncertainties in the values of $d \bar{\sigma}_{1}(E)$ arise from the $\sim 9 \%$ statistical uncertainty in $N^{\prime}(E)$, the $25 \%$ uncertainty in $d \sigma_{2}(E)$, the uncertainty in $F_{2}=0.185 \pm 0.040$, and the $10 \%$ uncertainty in $d \bar{\sigma}_{1}(16.5 \mathrm{eV})$.
*Present address: Department of Physics, University of Virginia, Charlottesville, VA 22901.

†Present address: Measurex Corporation, Cupertino, CA 95014.

¥Present address: Department of Physics, University of Missouri-Rolla, Rolla, MO 65401.

§Present address: AT\&T Bell Laboratories, Holmdel, NJ 07733.

${ }^{1}$ V. W. Hughes, in Proceedings of the Fifth International Conference on High Energy Accelerators (Comitato Nazionale per l'Energia Nucleare, Rome, Italy, 1966), p. 531.

${ }^{2}$ W. Raith, in Atomic Physics, edited by B. Bederson, V. W. Cohen, and F. M. J. Pichanick (Plenum, New York, 1969), p. 389; M. S. Lubell, in Atomic Physics 5, edited by R. Marrus, M. Prior, and H. Shugart (Plenum, New York, 1977), p. 325.

${ }^{3}$ E. Fues and H. Hellmann, Z. Phys. 31, 465 (1930).

${ }^{4}$ R. L. Long, Jr., W. Raith, and V. W. Hughes, Phys. Rev. Lett. 15, 1 (1965):

${ }^{5}$ V. W. Hughes, R. L. Long, Jr., M. S. Lubell, M. Posner, and W. Raith, Phys. Rev. A 5, 195 (1972).

${ }^{6} \mathrm{G}$. Baum, in Polarization Phenomena in Nuclear Physics-1980 (Fifth International Symposium, Santa Fe), Proceedings of the Fifth International Symposium on Polarization Phenomena in Nuclear Physics, edited by G. G. Ohlson, R. E. Brown, N. Jarmie, M. W. McNaughton, and G. M. Hale, (AIP, New York, 1981), p. 785.

${ }^{7}$ R. J. Celotta and D. T. Pierce, Adv. At. Mol. Phys. 16, 102 (1980).

${ }^{8}$ See, for example, P. S. Cooper et al., Phys. Rev. Lett. 34, 1589 (1975); 42, 1386 (1979); M. J. Alguard et al., ibid. 37, 1258 (1976); 37, 1261 (1976); G. Baum et al., ibid. 45, 2000 (1980); 51, 1135 (1983); W. Atwood et al., Phys. Rev. D 18, 2223 (1978); C. Y. Prescott et al., Phys. Lett. 77B, 347 (1978); 84B, 524 (1979).

${ }^{9}$ P. A. Souder et al., in High Energy Spin Physics-1982 (Brookhaven National Laboratory), Proceedings of the Fifth International High Energy Spin Symposium, edited by G. M. Bunce (AIP, New York, 1983), p. 574.

${ }^{10}$ D. T. Pierce and R. J. Celotta, Adv. Electron. Electron Phys. 56, 219 (1981).

${ }^{11}$ D. T. Pierce and R. J. Celotta, J. Vac. Sci. Technol. A 1, 1119 (1983).

${ }^{12}$ G. Baum, in Trends in Physics 1981, edited by I. A. Dorobantu (Central Institute of Physics, Bucharest, 1982), p. 460.

${ }^{13}$ G. F. Hanne, Phys. Rep. 95, 95 (1983).

${ }^{14} \mathrm{~W}$. Raith, in Proceedings of the XIII International Conference on the Physics of Electronic and Atomic Collisions, Berlin, 1983, edited by J. Eichler, I. V. Hertel, and N. Stolterfoht (North-Holland, Amsterdam, 1984), p. 107.

${ }^{15}$ G. F. Hanne and J. Kessler, Phys. Rev. Lett. 33, 341 (1974); in Electron and Photon Interactions with Atoms, edited by $\mathrm{H}$. Kleinpoppen and M. R. C. McDowell (Plenum, New York, 1976), p. 445; J. Phys. B 9, 791 (1976); G. F. Hanne, ibid. 9, 805 (1976).

${ }^{16}$ M. J. Alguard, V. W. Hughes, M. S. Lubell, and P. F. Wainwright, Phys. Rev. Lett. 39, 334 (1977).

${ }^{17}$ P. F. Wainwright, M. J. Alguard, G. Baum, and M. S. Lubell, Rev. Sci. Instrum. 49, 571 (1978).
${ }^{18}$ M. S. Lubell, in Coherence and Correlation in Atomic Collisions, edited by $\mathbf{H}$. Kleinpoppen and J. F. Williams (Plenum, New York, 1980), p. 663.

${ }^{19}$ D. Hils and H. Kleinpoppen, J. Phys. B 11, L283 (1978); D. Hils, W. Jitschin, and H. Kleinpoppen, ibid. 15, 3347 (1982).

${ }^{20}$ O. Berger, J. Kessler, K. J. Kollath, R. Möllenkamp, and W. Wübker, Phys. Rev. Lett. 46, 768 (1981); K. Bartschat, G. F. Hanne, A. Wolcke, and J. Kessler, ibid. 47, 997 (1981); W. Wübker, R. Möllenkamp, and J. Kessler, ibid. 49, 272 (1982).

${ }^{21}$ G. Baum, E. Kisker, W. Raith, W. Schröder, U. Sillmen, and D. Zenses, J. Phys. B 14, 4377 (1981).

${ }^{22}$ M. H. Kelley, W. T. Rogers, R. J. Celotta, and S. R. Mielczarek, Phys. Rev. Lett. 51, 2191 (1983).

${ }^{23}$ G. D. Fletcher, M. J. Alguard, T. J. Gay, V. W. Hughes, C. W. Tu, P. F. Wainwright, M. S. Lubell, W. Raith, and F. C. Tang, Phys. Rev. Lett. 48, 1671 (1982); G. D. Fletcher, Ph.D. thesis, Yale University, 1983.

${ }^{24}$ T. J. Gay, G. D. Fletcher, M. J. Alguard, V. W. Hughes, P. F. Wainwright, and M. S. Lubell, Phys. Rev. A 26, 3664 (1982).

${ }^{25}$ W. L. Fite and R. T. Brackmann, Phys. Rev. 112, 1141 (1958).

${ }^{26}$ W. Lichten and S. Schultz, Phys. Rev. 116, 1132 (1959); W. E. Kauppila, W. R. Ott, and W. L. Fite, Phys. Rev. A 1, 1099 (1970); H. Koschmieder, V. Raible, and H. Kleinpoppen, ibid. 8, 1365 (1973); J. F. Williams, J. Phys. B 9, 1519 (1976).

${ }^{27}$ P. J. O. Teubner, C. R. Lloyd, and E. Weigold, Phys. Rev. A 9, 2552 (1974); C. R. LLoyd, P. J. O. Teubner, E. Weigold, and B. R. Lewis, ibid. 10, 175 (1974).

28J. F. Williams, J. Phys. B 7, L56 (1974); 8, 1683 (1975); 8, 2191 (1975).

${ }^{29}$ J. F. Williams, in Electron and Photon Interactions with Atoms, edited by H. Kleinpoppen and M. R. C. McDowell (Plenum, New York, 1976), p. 309.

30J. Callaway and J. F. Williams, Phys. Rev. A 12, 2312 (1975).

${ }^{31}$ K. Rubin, B. Bederson, M. Goldstein, and R. E. Collins, Phys. Rev. 182, 201 (1969).

${ }^{32}$ H. Kleinpoppen, Phys. Rev. A 3, 2015 (1971).

${ }^{33}$ J. Kessler, Polarized Electrons (Springer, Berlin, 1976).

${ }^{34} \mathrm{See}$, for example, various contributions to Coherence and Correlation in Atomic Collisions, edited by H. Kleinpoppen and J. F. Williams (Plenum, New York, 1980).

${ }^{35}$ N. F. Mott and H. S. W. Massey, The Theory of Atomic Collisions, 3rd ed. (Oxford University, London, 1965).

36R. Peterkop, Proc. Phys. Soc. London Sect. A 77, 1220 (1961).

${ }^{37} \mathrm{~S}$. Geltman, Topics in Atomic Collision Theory (Academic, New York, 1969).

${ }^{38}$ P. G. Burke, Potential Scattering in Atomic Physics (Plenum, New York, 1977).

${ }^{39}$ C. J. Joachain, Quantum Collision Theory (North-Holland, Amsterdam, 1979).

${ }^{40}$ B. H. Bransden, Atomic Collision Theory, 2nd ed. (Benjamin/Cummings, Reading, Mass., 1983).

${ }^{41}$ P. G. Burke and J. F. Williams, Phys. Rep. 34, 325 (1977).

${ }^{42}$ F. W. Byron, Jr. and C. J. Joachain, Phys. Rep. 34, 233 (1977).

${ }^{43}$ B. H. Bransden and M. R. C. McDowell, Phys. Rep. 30, 207 (1977); 46, 249 (1978).

44J. Callaway, Phys. Rep. 45, 89 (1978); Adv. Phys. 29, 771 
(1980).

${ }^{45}$ F. T. Chan, M. Lieber, G. Foster, and W. Williamson, Jr., Adv. Electron. Electron Phys. 49, 133 (1979).

${ }^{46}$ C. Schwartz, Phys. Rev. 124, 1468 (1961).

${ }^{47}$ I. Shimamura, J. Phys. Soc. Jpn. 30, 1702 (1971).

${ }^{48}$ M. R. H. Rudge, J. Phys. B 8, 940 (1975).

${ }^{49}$ D. Register and R. T. Poe, Phys. Lett. 51A, 431 (1975).

${ }^{50}$ R. L. Armstead, Phys. Rev. 171, 91 (1968).

51J. N. Das and M. R. H. Rudge, J. Phys. B 9, L131 (1976).

${ }^{52}$ M. K. Gailitis, in Proceedings of the Fourth International Conference on Electronic and Atomic Collisions, Abstracts of Papers, Université Laval, Quebec, 1965 (Science Bookcrafters, Hastings-on-Hudson, New York, 1965), p. 10.

53J. Callaway, Phys. Lett. 65A, 199 (1978).

${ }^{54}$ A. E. Kingston and H. R. J. Walters, J. Phys. B 13, 4633 (1980).

${ }^{55}$ B. H. Bransden and J. P. Coleman, J. Phys. B 5, 537 (1972); B. H. Bransden, J. P. Coleman, and J. Sullivan, ibid. 5, 546 (1972).

${ }^{56}$ K. H. Winters, C. D. Clark, B. H. Bransden, and J. P. Coleman, J. Phys. B 7, 788 (1974).

${ }^{57}$ R. Vanderpoorten, J. Phys. B 8, 926 (1975).

${ }^{58}$ B. H. Bransden and C. J. Noble, J. Phys. B 9, 2461 (1976).

${ }^{59}$ M. R. C. McDowell, Comments At. Mol. Phys. 9, 127 (1980).

${ }^{60}$ T. Scott and B. H. Bransden, J. Phys. B 14, 2277 (1981).

${ }^{61}$ F. W. Byron, Jr. and C. J. Joachain, J. Phys. B 14, 2429 (1981).

${ }^{62}$ F. W. Byron, Jr. and C. J. Joachain, Phys. Rev. A 8, 1267 (1973); J. Phys. B 7, L212 (1974).

${ }^{63}$ F. W. Byron, Jr. and C. J. Joachain, J. Phys. B 10, 207 (1977).

${ }^{64}$ F. W. Byron, Jr., C. J. Joachain, and R. M. Potvliege, J. Phys. B 14, L609 (1981); 15, 3915 (1982).

${ }^{65}$ V. Franco, Phys. Rev. Lett. 20, 709 (1968).

${ }^{66}$ G. Foster and W. Williamson, Jr., Phys. Rev. A 13, 936 (1976).

${ }^{67}$ G. Khayrallah, Phys. Rev. A 16, 2064 (1976).

${ }^{68}$ T. T. Gien, J. Phys. B 9, 3203 (1976); Phys. Rev. A 16, 123 (1977).

${ }^{69}$ T. Ishihara and J. C. Y. Chen, Phys. Rev. A 12, 370 (1975).

${ }^{70}$ A.-L. Sinfailam and J. C. Y. Chen, Phys. Rev. A 5, 1218 (1972); J. C. Y. Chen, L. Hambro, A.-L. Sinfailam, and K. T. Chung, ibid. 7, 2003 (1973).

${ }^{71}$ P. G. Burke and H. M. Schey, Phys. Rev. 126, 147 (1962); 126, 163 (1962); P. G. Burke and K. Smith, Rev. Mod. Phys. 34, 458 (1962).

72P. G. Burke, H. M. Schey, and K. Smith, Phys. Rev. 129, 1258 (1963).

73P. G. Burke, S. Ormonde, and W. Whitaker, Proc. Phys. Soc. London 92, 319 (1967).

${ }^{74}$ A. J. Taylor and P. G. Burke, Proc. Phys. Soc. London 92, 336 (1967).

${ }^{75}$ P. G. Burke, D. F. Gallaher, and S. Geltman, J. Phys. B 2, 1142 (1969).

76J. Callaway and J. W. Wooten, Phys. Rev. A 9, 1924 (1974).

${ }^{77}$ W. C. Fon, P. G. Burke, and A. E. Kingston, J. Phys. B 11, 521 (1978).

${ }^{78}$ A. Temkin and J. C. Lamkin, Phys. Rev. 121, 788 (1961).

${ }^{79}$ R. J. Drachman and A. Temkin, in Case Studies in Atomic Collision Physics II, edited by E. W. McDaniel and M. R. C. McDowell (North-Holland, Amsterdam, 1972), p. 399.

${ }^{80}$ R. K. Nesbet, Phys. Rev. 179, 60 (1969); R. K. Nesbet and R. S. Oberoi, Phys. Rev. A 6, 1855 (1972).

${ }^{81}$ G. J. Seiler, R. S. Oberoi, and J. Callaway, Phys. Rev. A 3, 2006 (1971).
82P. G. Burke, A. Hibbert, and W. D. Robb, J. Phys. B 4, 153 (1971).

${ }^{83}$ W. C. Fon, K. A. Berrington, P. G. Burke, and A. E. Kingston, J. Phys. B 14, 1041 (1981).

${ }^{84}$ A. K. Kingston and H. R. J. Walters, Comments At. Mol. Phys. 11, 177 (1982).

${ }^{85}$ M. R. H. Rudge, Rev. Mod. Phys. 40, 564 (1968).

${ }^{86}$ V. I. Ochkur, Zh. Eksp. Teor. Fiz. 45, 734 (1963) [Sov. Phys.-JETP 18, 503 (1964)].

${ }^{87}$ M. R. H. Rudge, Adv. At. Mol. Phys. 9, 47 (1973).

${ }^{88}$ R. N. Madan, Phys. Rev. A 11, 1968 (1975); 12, 2631 (1975).

${ }^{89}$ G. Foster and W. Williamson, Jr., Phys. Rev. A 13, 2023 (1976).

${ }^{90}$ B. H. Bransden, M. R. C. McDowell, C. J. Noble, and T. Scott, J. Phys. B 9, 1301 (1976).

${ }^{91}$ D. P. Dewangan, Phys. Lett. 56A, 279 (1976).

${ }^{92}$ H. R. J. Walters, J. Phys. B 13, L749 (1980).

${ }^{93}$ V. Franco and A. M. Halpern, Phys. Rev. A 21, 1118 (1980).

${ }^{94}$ B. van Wingerden, E. Weigold, F. J. de Heer, and K. J. Nygaard, J. Phys. B 10, 1345 (1977).

${ }^{95}$ A. M. Ermolaev and H. R. J. Walters, J. Phys. B 12, L779 (1979).

${ }^{96}$ G. H. Wannier, Phys. Rev. 90, 817 (1953).

97J. W. McGowan and E. M. Clarke, Phys. Rev. 167, 43 (1968).

${ }^{98}$ S. Cvejanović and F. H. Read, J. Phys. B 7, 1841 (1974).

${ }^{99}$ J. B. Donahue et al., Phys. Rev. Lett. 48, 1538 (1982).

${ }^{100}$ A. R. P. Rau, Phys. Rev. A 4, 207 (1971).

${ }^{101}$ R. Peterkop, J. Phys. B 4, 513 (1971).

${ }^{102}$ T. A. Roth, Phys. Rev. A 5, 476 (1971).

${ }^{103}$ H. Klar and W. Schlect, J. Phys. B 9, 1699 (1976).

${ }^{104}$ C. H. Greene and A. R. P. Rau, Phys. Rev. Lett. 48, 533 (1982); J. Phys. B 16, 99 (1983). See also A. D. Stauffer, Phys. Lett. 91A, 114 (1982).

${ }^{105}$ A. Temkin, Phys. Rev. Lett. 49, 365 (1982); Comments At. Mol. Phys. 11, 287 (1982).

${ }^{106}$ E. W. Rothe, L. L. Marino, R. H. Neynaber, and S. M. Trujillo, Phys. Rev. 125, 582 (1962).

${ }^{107} \mathrm{H}$. Frauenfelder and A. Rossi, in Methods of Experimental Physics, edited by L. C. L. Yuan and C. S. Wu (Academic, New York, 1963), Vol. 5, Pt. B, p. 214.

${ }^{108}$ G. D. Fletcher, T. J. Gay, and M. S. Lubell, Rev. Sci. Instrum. (to be published).

${ }^{109}$ U. Fano, Phys. Rev. 178, 131 (1969); also see addendum, Phys. Rev. 184, 250 (1969).

${ }^{110}$ N. F. Mott, Proc. R. Soc. London, Ser. A 124, 425 (1929); 135, 429 (1932).

${ }^{111}$ H. M. Brash, D. M. Campbell, P. S. Farago, A. G. A. Rae, H. Chr. Siegmann, and J. S. Wykes, Proc. R. Soc. Edinburgh Ser. A 68, Pt. II, 158 (1969).

${ }^{112}$ G. Baum, M. S. Lubell, and W. Raith, Phys. Rev. A 5, 1073 (1972).

113J. F. Williams and B. A. Willis, J. Phys. B 8, 1670 (1975).

${ }^{114}$ R. D. Dubois and M. E. Rudd, J. Phys. B 9, 2657 (1976).

${ }^{115}$ S. K. Srivastava, H. Tanaka, A. Chutjian, and S. Trajmar, Phys. Rev. A 23, 2156 (1981).

${ }^{116}$ S. K. Srivastava, A. Chutjian, and S. Trajmar, J. Chem. Phys. 63, 2659 (1975).

${ }^{117}$ T. W. Shyn and W. E. Sharp, Phys. Rev. A 24, 1734 (1981).

${ }^{118}$ H. D. Zeman, K. Jost, and S. Gilad, Rev. Sci. Instrum. 42, 485 (1971).

${ }^{119}$ Early electron polarization measurements performed with this apparatus (Refs. 16-18) did not include corrections due either to elastic scattering from the Formvar backing or to the polarization dependence of the inelastic background. The 
electron polarization, when corrected for these effects, was found to be increased by $12 \%$ from the value determined during the early $A_{90^{\circ}}(1 S \rightarrow 1 S$ ) measurements (Ref. 18), and was found to be increased by $5 \%$ from the value obtained during the early $A_{I}$ measurements (Ref. 16). The values of $A_{90^{\circ}}(1 S \rightarrow 1 S)$ and $A_{I}$ from the early work presented in Sec. $\mathrm{V}$ contain these corrections.

${ }^{120}$ Shin-R Lin, Phys. Rev. 133, A965 (1964).

${ }^{121} \mathrm{G}$. Holzwarth and H. J. Meister, Nucl. Phys. 59, 56 (1964).

1223M Company, Part No. 105 UV WRMR.

${ }^{123}$ E. H. Kennard, Kinetic Theory of Gases (McGraw-Hill, New York, 1938), p. 442.

${ }^{124}$ H. D. Young, Statistical Treatment of Experimental Data (McGraw-Hill, New York, 1962).

${ }^{125} \mathrm{~S}$. Trajmar (private communication).

${ }^{126} \mathrm{The} 8.3-\mathrm{eV}$ result was originally assigned an energy of $7.7 \mathrm{eV}$, while the $10.0-\mathrm{eV}$ result represents a weighted average of the early results assigned energies of 10.4 and $10.5 \mathrm{eV}$. The early measurement obtained at $7.5 \mathrm{eV}$ was discarded, since it comprised data taken for only one hydrogen polarization direction at a time when systematic effects were not well understood.

${ }^{127}$ T. F. O'Malley, L. Spruch, and L. Rosenberg, J. Math. Phys. 2, 491 (1961).

${ }^{128}$ B. L. Scott, Phys. Rev. 140, A699 (1965).

${ }^{129}$ S. Geltman, Phys. Rev. 119, 1283 (1960).

${ }^{130} \mathrm{G}$. Khayrallah, in Eleventh International Conference on the Physics of Electronic and Atomic Collisions, Abstracts of Contributed Papers, edited by K. Takayanagi and N. Oda (North-Holland, Amsterdam, 1979), p. 114.

${ }^{131} \mathrm{G}$. Baum (private communication).

${ }^{132}$ M. R. H. Rudge and M. J. Seaton, Proc. R. Soc. London, Ser. A 283, 262 (1965).

${ }^{133}$ R. K. Peterkop, Zh. Eksp. Teor. Fiz. 41, 1938 (1961) [Sov. Phys._JETP 14, 1377 (1962)].

${ }^{134}$ S. Geltman, M. R. H. Rudge, and M. J. Seaton, Proc. Phys. Soc. London 81, 375 (1963).
135J. E. Golden and J. H. McGuire, Phys. Rev. Lett. 32, 1218 (1974).

${ }^{136}$ M. R. H. Rudge and S. B. Schwartz, Proc. Phys. Soc. London 88, 563 (1966).

${ }^{137}$ V. I. Ochkur, Zh. Eksp. Teor. Fiz. 47, 1746 (1964) [Sov. Phys.-JETP 20, 1175 (1965)].

${ }^{138}$ D. F. Gallaher, J. Phys. B 7, 362 (1974).

${ }^{139}$ M. R. H. Rudge, J. Phys. B 11, L149 (1978).

${ }^{140}$ A. Vasilakis, F. C. Tang, and M. S. Lubell, Bull. Am. Phys. Soc. 26, 1296 (1981); A. Vasilakis et al., in Thirteenth International Conference on the Physics of Electronic and Atomic Collisions, Abstracts of Contributed Papers, edited by $\mathbf{J}$. Eichler, W. Fritsch, I. V. Hertel, N. Stolterfoht, and U. Wille (North-Holland, Amsterdam, 1983), p. 145.

${ }^{141}$ A. J. Dixon, S. T. Hood, and E. Weigold, Phys. Rev. Lett. 40, 1262 (1978); S. T. Hood, E. Weigold, and A. J. Dixon, J. Phys. B 12, 631 (1979); E. Weigold, in Coherence and Correlation in Atomic Collisions, Ref. 34, p. 59.

${ }^{142}$ M. T. Hollywood, A. Crowe, and J. F. Williams, in Coherence and Correlation in Atomic Collisions, Ref. 34, p. 97; J. F. Williams, J. Phys. B 14, 1197 (1981).

${ }^{143}$ J. Slevin, M. Eminyan, J. M. Woolsey, G. Vassilev, and H. Q. Porter, J. Phys. B. 13, L341 (1980); J. Slevin et al., Phys. Rev. A 26, 1344 (1982).

${ }^{144}$ D. T. Pierce and F. Meier, Phys. Rev. B 13, 5484 (1976).

${ }^{145}$ D. T. Pierce et al., Rev. Sci. Instrum. 51, 478 (1980).

${ }^{146} \mathrm{~J}$. Slevin and W. Stirling, Rev. Sci. Instrum. 52, 1780 (1981).

${ }^{147}$ For a review, see S. Trajmar, D. F. Register, and A. Chutjian, Phys. Rep. 97, 219 (1983).

${ }^{148}$ F. Linder and H. Schmidt, Z. Naturforsch. A 26, 1603 (1971).

${ }^{149}$ S. Trajmar, D. G. Truhlar, J. K. Rice, and A. Kuppermann, J. Chem. Phys. 52, 4516 (1970).

${ }^{150}$ S. K. Srivastava, R. I. Hall, S. Trajmar, and A. Chutjian, Phys. Rev. A 12, 1399 (1975).

${ }^{151}$ D. F. Register, S. Trajmar, and S. K. Srivastava, Phys. Rev. A 21, 1134 (1980). 